\title{
A Superspace Formalism for the Electromagnetism of Generic Nonlocal Continuous Metamaterials: Principal Structures and Applications
}

This paper was downloaded from TechRxiv (https://www.techrxiv.org).

\section{LICENSE}

CC BY 4.0

SUBMISSION DATE / POSTED DATE

$27-01-2021 / 28-01-2021$

\section{CITATION}

Mikki, Said (2021): A Superspace Formalism for the Electromagnetism of Generic Nonlocal Continuous Metamaterials: Principal Structures and Applications. TechRxiv. Preprint.

https://doi.org/10.36227/techrxiv.13649609.v1

$\mathrm{DOI}$

10.36227/techrxiv.13649609.v1 


\title{
A Superspace Formalism for the Electromagnetism of Generic Nonlocal Continuous Metamaterials: Principal Structures and Applications
}

\author{
Said Mikki ${ }^{1}$
}

\begin{abstract}
An alternative to conventional spacetime is rigoursly formulated for nonlocal electromagnetism using the general concept of fiber bundle superspace. We develop in increasing complexity the concept of nonlocality starting from general considerations, going through spatial dispersion, and ending up with a broad formulation that unveils the link between general topology and electromagnetic nonlocality in generic material media. It is shown that electromagnetic nonlocality naturally leads to a Banach (vector) bundle structure serving as an enlarged space (superspace) inside which electromagnetic processes take place. The added structures, essentially fibered spaces, model the topological microdomains of electromagnetic nonlocality and provide a fine-grained geometrical picture of field-matter interactions in nonlocal metamaterials. We utilize standard techniques in the theory of smooth manifolds to construct the Banach bundle structure by paying careful attention to the relevant physics. The electromagnetic response tensor is then reformulated as a superspace bundle homomorphism and the various tools needed to proceed from the local topology of microdomains to global domains are developed. Concrete examples quantitatively illustrating the microdomain structure in nonlocal semiconductors are provided. Discussions of other applications are given, including shedding some light on why nonlocal electromagnetic materials often require additional boundary conditions or extra input from microscopic theory in comparison with local electromagnetism. ${ }^{1}$
\end{abstract}

Index Terms-Nonlocal metamaterials, multiscale structures, fiber bundles, superspace.

\section{INTRODUCTION}

In classical electromagnetic (EM) theory, there are no nonlocal interactions or phenomena in vacuum because Maxwell's equations, which capture the ultimate content of the physics of electromagnetic fields, are essentially local differential equations [1]. An effect applied at point $\mathbf{r}$ in space will first be felt at the same location but then spread or propagate slowly into the infinitesimally immediate neighborhood. ${ }^{2}$ Long-term disturbances such as electromagnetic waves propagate through both vacuum and material media by cascading these infinitesimal perturbations in outward directions (rays or propagation

\footnotetext{
${ }^{1}$ The author is with Zhejiang University/University of Illinois at UrbanaChampaign (ZJU-UIUC) Institute, Engineering Building, 718 East Haizhou Road Haining, Zhejiang, China; Royal Military College of Canada, 13 General Crerar Crescent, Kingston, Ontario K7K 7B4, Canada. E-mail address: said.m.mikki@gmail.com.

${ }^{2}$ It is commonly accepted that Aharonov-Bohm effects, which lead to observable nonlocal electrodynamic effects, have their origin in quantum physics. In this paper we focus on classical field theory described using phenomenological models. Some authors, however, suggest that classical electromagnetism may induce nonlocal effects but such topic is outside the scope of the present paper.
}

paths) emanating from the time-varying point source that originated the whole process. On the other hand, nonlocal interactions differ from this vacuum-like picture in allowing fields applied at position $\mathbf{r}^{\prime}$ to influence the medium at different location $\mathbf{r}$, i.e., a location that is not infinitesimally close to the source position $\mathbf{r}^{\prime}$. The distance $\left|\mathbf{r}-\mathbf{r}^{\prime}\right|$ could be very small in most media (and certainly zero in vacuum), but in some types of materials, the so-called nonlocal media, observable response can be found such that the "radius of nonlocality" $\left|\mathbf{r}-\mathbf{r}^{\prime}\right|$ is appreciably different from zero [2][4]. This is the core idea of nonlocality in electromagnetic metamaterials. The research field concerned with the study of the electromagnetism of such material domains is called nonlocal electromagnetism/electromagnetics/electrodynamics. ${ }^{3}$ This paper introduces a comprehensive new general theory for this emerging discipline together with a series of selected applications.

The main goal of the present work is to explore at a very general level the conceptual and mathematical foundations of nonlocality in connection with applied electromagnetic metamaterials (MTMs). ${ }^{4}$ Our approach is conceptual and theoretical with the main focus on understanding the mathematical foundations of the subject at a very broad level. Indeed, while a massive amount of numerical and experimental data on all types of nonlocal materials abound in a literature that goes back to as early as the 1950s, the purpose of the present paper is attaining some clear understanding of the essentials of the subject, particularly in connection with the ability to build a very general superspace formalism for nonlocal electromagnetism without restricting the formalism first to particular classes of materials such as metals, plasmas, or semiconductors.

The superspace formalism has a long history in physics, mathematical physics, and mathematics (see Remark I.1). It will be shown below that nonlocal electromagnetism appears to lead very naturally to a reformulation of its essential configuration space by upgrading the conventional space-time or frequency-space to a larger superspace in which the former spaces serve as base spaces for the new (larger) superspace. Such reconsideration of the fundamental structure of the prob-

\footnotetext{
${ }^{3}$ Extensive literature survey on nonlocal electromagnetism is given in Sec. II-A and Remark III.3.

${ }^{4}$ Currently, there is an interest within applied physics and engineering in harnessing nonlocal media as a new generation of metamaterials for use in various settings, e.g., optical devices, energy control, antennas, circuit systems, etc., see Sec. II-A
} 
lem may help foster future numerical methods and potential applications as will be discussed later.

Remark I.1. (Superspace Concepts in Other Fields) The concept of superspace is not new and has been proposed in several fields in both physics and mathematics. For a brief but general view on the definition of superspaces, see [5]. For example applications, various superspaces have been proposed as fundamental structures in quantum gravity [6], [7], which are frequently infinite dimensional. Superspace concepts are also now extensively researched in quantum field theory and the standard model of particle physics, e.g., see [8][10]. In general, dealing with topics such as supergravity, supersymmetry, superfields, superstrings, and noncommutative geometry often requires the use of one superspace formalism or another [8]. More related to the subject of nonlocal MTMs is the original superspace concept introduced earlier for the analysis of deformed crystal [11] and subsequently utilized for fundamental investigations of EM nonlocality in incommensurate (IC) superstructures in insulators [12]. Such modulatedstructure materials possess spaces with dimensions greater than spacetime [13]. Nevertheless, for fairly concrete models one may exploit group theory to construct finite-dimensional (dimension $>4$ ) approximations of them. The general theory of superspace formalisms in quasiperiodic crystals is presented in [14]. Other examples from condensed-matter physics where superspace methods where applied include mesoscopic superconductivity [15]. In mathematics and mathematical physics, where the concept itself originated, a notable recent example of the superspace concept includes sheaves, which are used in differential and algebraic topology and algebraic geometry and have numerous applications in physics [16]-[18].

The key motivation for our superspace approach is based on explicating the subtle but often overlooked difference between infinitesimal interactions, which characterize local electromagnetism, and interactions occurring in small topological neighborhoods around the observation point. We believe that this topological difference has not received the attention it deserves in the growing theoretical and methodological literature on nonlocal media. In particular, the author believes that a majority of present approaches to nonlocal metamaterials conflate the topologically local (but EM nonlocal) domain of small neighborhoods and global domains. However, general topology and much of modern mathematical physics is based on clearly distinguishing the last two topological levels. It turns out that the standard formalism of local electromagnetism, which is based on spacetime points and their differential but not topological neighborhoods as the basic configuration space of the problem, is not the most natural or convenient framework for formulating the electromagnetism of nonlocal materials. This is mainly because the physicsbased domain of electromagnetic nonlocality (to be defined precisely below), which captures the effective region of fieldmatter nonlocal interactions, is not usually built into the mathematical formalism of classical boundary value problems in applied electromagnetism. By investigating the subject from a new perspective, it will be shown that a natural space for conducting nonlocal metamaterials research is the vector bundle structure, more specifically, a Banach bundle [19] where every element in the fiber superspace is a vector field on the entire domain of nonlocality.

The main result of this paper is that every generic nonlocal domain can be topologically described by a superspace comprised of a Banach (infinite-dimensional) vector bundle $\mathcal{M}$. If two materials described by their corresponding vector bundles $\mathcal{M}_{1}$ and $\mathcal{M}_{2}$ are juxtaposed, then one may use topological methods to combine them and to compare their topologies. The present paper's focus is mainly on the first part, i.e., how to construct the material bundle $\mathcal{M}$. That is, the derivation of the various vector bundle structures starting from a generic phenomenological model of electromagnetic nonlocality is the main contribution of the present work. It is the hope that the superspace theory will stimulate new approaches to computational EM by adopting methods from computational topology and differential topology for solving challenging problems in complex material domains.

\section{REVIEW OF NONLOCAL ELECTROMAGNETISM AND AN OUtLINE OF THE PRESENT WORK}

\section{A. Survey of the Literature on Nonlocal Metamaterials}

We first provide a non-exhaustive and selective review of the development of nonlocal electromagnetic materials research. ${ }^{5}$ Some of the physical phenomena that cannot be understood using local electromagnetic theory include spatial dispersion effects [20], extreme negative group velocity and negative refraction [21], [22], new diffraction behaviour in optical beams [23], superconductivity [24], natural optical activity [4], [25], [26], non-Planck equilibrium radiation formulas in nonlocal plasma [27]. Outside electromagnetism but within wave phenomena, there also exists processes that cannot be fully accounted for through simple local material models, for instance, we mention phase transitions, Casimir force effects [28], and streaming birefringence [29]. By large, spatial dispersion has attracted most of the attention of the various research communities working on nonlocal electromagnetic materials. Indeed, few book-length researches on spatial dispersion already exist in literature, most notably [2]-[4], [20].

The majority of published research on nonlocal media and nonlocal electromagnetism tend to focus on applications and specialized materials (see references quoted below.) Few Exceptions include investigations attempting to approach the subject at a more general level. For example, from the perspective of general thermodynamics, see [29], [30]. Some of the topics reexamined within the framework of a general nonlocal fieldmatter interaction theory include the applicability of optical reciprocity theorems [31]-[34], energy/power balance [35], quantization [36]-[38], operator methods [39], extension of spatial dispersion to include inhomogeneous media [12], and alternative formulations of spatial dispersion in terms of the Jones calculus [40].

The bulk of the available research on nonlocality is concentrated in the very large area of general field-matter interactions. There already exists a well-attested body of research

\footnotetext{
${ }^{5}$ More information and proposals regarding engineering applications are given in Sec. IX-B, where additional references can be found.
} 
on nonlocality in metals based on various phenomenological approaches, e.g., see [41] for a general review. Nonlocality has also been investigated extensively in dielectric media, for example semiconductors [20], [42]. A comprehensive recent review of nonlocality in crystal structures is provided in [43], which updates the classic books [4], [44]. Moreover, numerous researches conducted within condensed-matter physics and material science implicitly or explicitly assume that nonlocality is essentially based on microscopic (hence quantum) processes, and develop an extensive body of work where the spatially dispersive dielectric tensor is deployed as the representative constitutive material relation [45]-[49]. On the other hand, one can also treat nonlocality without resort to spatial dispersion by modeling certain classes of material media as periodic structures [50] where the susceptibility tensor is derived from the symmetry of the overall structure [45], [51], [52] or from the lattice dynamics approach [53], [54].

For solving nonlocal problems, several methods exists in order to deal with the lack of universal model at the interface between a nonlocal material and another medium. The Additional Boundary Condition (ABC) approach adjoins new boundary conditions to the standard Maxwell's equations in order to account for additional waves excited at the interface, which otherwise would not be explained by the standard local theory [4]. All ABC formulations are inherently model-specific since they assume particular types of nonlocal media or postulate specific ABCs based on the physics and applications, e.g., see [44], [55]-[59]. We note that such ABC formalisms are not inevitable since there exists several boundary-condition free formulations, e.g., see [46], [47], [51].

In the field of computational methods, a number of discretization strategies for dealing with full-wave analysis in the presence of nonlocal materials were proposed. For example, an FDTD method was proposed in [60] to deal with metallic spatially dispersive objects. Surface integral equations for nonlocal plasmonic materials were also proposed in [61], [62], with discretizstion done using the RWG basis functions. Moreover, specialized methods were proposed for various possible scenarios involving nonlocal field-matter interactions, such as nonlocal dielectric profile retrieval from measurable data [63], iterative solutions of nonlocal wave equations [64], [65], applications of the derivative expansion method to nonlocal plasma analysis [66], application of Kramers-Kronig relation method [67], application of the Pade approximation to homogenization [68],

The idea of exploiting nonlocality to design and develop a new generation of metamaterials (MTMs) exhibiting novel EM behaviour has also received a revival in recent years, though the basic concept in itself is not completely new, going back to at least [4] and possibly earlier. Recent examples of research focused in explicating nonlocal behaviour to harness the associated new physics include spatial dispersion in photonic crystals [69], wire media [70]-[73], semiconductor nanoparticles [74]-[77], optically nonlinear liquids [78], hyperbolic metamaterials [79], layered dielectric-metal structures [80], [81] and thin films [82], plasma-based metamaterials [83][85], quantum wells [86], soliton interactions with matter [87]-
[92], superconducting films [93] and circuits [94], plasmonic devices and structures [95]-[97], nanocubes [98], cloaking [99], Chern metamaterials [100] and superconductors [101], dispersion management profiles [22], [102], biomedical applications in materials [103], and nonlocal uniaxial metamaterials [104].

Numerous homogenization theories for nonlocal MTM with averaging operations considered over multiple spatial scales have been reported, e.g., see [72], [105]-[108]. We note that the subject of electromagnetic metamaterials (with or without nonlocality) is enormous and it is beyond the scope of this paper to even summarize the main papers in the field. However, most publications (till recently) have focused on non-spatially dispersive and local scenarios. This situation has began to change in recent years, and increasing numbers of reports appear to move from the old opinion that nonlocality is a "bug" to the more positive and fruitful perspective that nonlocality provides new dimensions to be exploited in metamaterial system design.

A particularly interesting direction of research in nonlocal media is the recent subject of topological photonics. The main idea was inspired by previous researches in Chern insulators and topological insulators [109], where the focus has been on electronic systems. There, it has already been observed that the nonlocal behaviour of the fermionic wavefunction may exhibit a rather interesting and nontrivial dependence on the entire configuration space of the system, in that case the momentum space (the wavevector $\mathbf{k}$ space). In addition to the already established role played by nonlocality in superconductors, quantum Hall effects are among the most intriguing physically observable phenomena that turned out to depend fundamentally on purely topological aspects of the electron wavefunction [24]. The major themes exhibited by electrons undergoing topological transition states include topological robustness of the excited edge (surface) states moving along a 2-dimensional interface under the influence of an external magnetic fields. More recently, it was proposed that the same phenomenon may apply to photons (electromagnetism) [110], where the key idea was to use photonic crystals to emulate the periodic potential function experienced by electrons in fermionic systems. However, since photons are bosons, transplanting the main theme of topological insulators into photons is not trivial and is currently generating great attention, see for example the extensive review article [111], which provides a literature survey of the field.

One of the most important applications of topological photonics is the presence of "edge states," which are topologically robust unidirectional surface waves excited on the interface between two metamaterials with topologically distinct invariants. Since edge states are immune to perturbations on the surface, they have been advocated for major new applications where topology and physics become deeply intertwined [112]. Topology can also be exploited to devise non-resonant metamaterials [113] and to investigate bifurcation transitions in media [114]. Another related exciting subject illustrating the synergy between topology, physics, and engineering is nonHermitian dynamics, especially in light of recent work related to the origin of surface waves [115], [116], which is now being 
considered as essentially non-trivial topological effect.

The previous old and recent directions of research all point toward a basic fact: topology and physics are destined to come closer to each other throughout the next decades. However, the unique feature in this convergence, though in itself is not totally new since Herman Weyl introduced topological thinking to physics in the 1920s, is the focus on material engineering applications, in our case metamaterials and topology-based devices. For that reason, we propose that in addition to the now mainstream approach to topological materials where the focus is on the global dependence of the wavefunction on momentum (Fourier) space, there is a need to consider how materials can be assigned a direct structure in the configuration space, i.e., space-time or space-frequency. Our key observation is that EM nonlocality requires gathering information at microdomains (small regions around every point where the response is nonlocal), then aggregating these microdomains together in order to arrive into a global topological structure. The fundamental insight coming from topology is precisely how this process of "moving from the local to the global" can be enacted. We have found that a very efficient method to do this is the natural formulation of the entire problem in terms of a fiber bundle. In other words, in contrast to most existing works on topological materials, we don't first solve Maxwell's equations to find the state function in the Fourier kspace then study topology over momentum space; instead, we work directly in spacetime (or space-frequency) and formulate the dual problem of the topology over a fiber bundle. It is the hope of the author that such new perspective may provide a complementary approach to the exciting subject of topological materials and help generate new insights into the physics and novel algorithms for the computation of suitable topological invariant characterizing complex material domains.

\section{B. An Outline of the Present Work}

Because of the complexity of the subject, and to make it more accessible to a wider audience involving physicists, engineers, and mathematicians, we have divided the argument into different stages with different flavors as follows. In Sec. III, we kick off our presentation by introducing a general review of electromagnetic nonlocality targeting a wide audience of mathematicians, physicists, engineers and applied scientist. The key ingredients of nonlocal metamaterials/materials are illustrated in Sec. III-A using an abstract excitation-response model. This is followed in Sec. III-B by a more detailed description of the special but important case of spatial dispersion, which tends to arise naturally in many investigations of nonlocal metamaterials. In Sec. IV, we begin the elucidation of the main topological ideas behind electromagnetic nonlocality, most importantly, the concept of EM nonlocality microdomains, which provides the key link between physics, material engineering, and topology in this paper. The various physical and mathematical structures are spelled out explicitly, followed in Sec. $\mathrm{V}$ by a more careful construction of a natural fiber bundle superspace structure that appears to satisfy simultaneously both the physical and mathematical requirements of EM nonlocality (Sec. V-A,V-B.) We then provide a key computational application of the proposed theory in Sec. $\mathrm{V}-\mathrm{C}$, where it is shown that the material response function is representable as a special fiber bundle homomorphism over the metamaterial base space. In this way, a more general map than linear operators in local EM is derived to provide mathematical foundations for future computational topological methods in which the bundle homomorphism is discretized instead of the linear operator itself. The fiber bundle superspace algorithm is summarized in Sec. VI where it is highlighted that the main data needed are the EM nonlocality microdomains, which come from physics. Otherwise, the entire construction of the superspace can proceeds using the procedure outlined. To illustrate how the above mentioned microdomain structure may be estimated in practice, we give in Sec. VII a computational example based on nonlocal semiconductors and also explore in depth the physical origin of nonlocality. Insights into the lack of general EM boundary conditions in nonlocal EM are provided in Sec. VIII based on the superspace formalism. In Sec. IX, various additional current and future applications to fundamental methods, applied physics, and engineering are outlined in brief form. Finally we end up with conclusion.

Some basic familiarity with vector bundles and Banach spaces is assumed, but essential definitions and concepts will be reviewed briefly within the main formulation and references where more background on vector bundles can be found will be pointed out. The paper intentionally avoids the strict theorem-proof format to make it accessible to a wider audience. Most of the time we give only proof sketches and leave out straightforward but lengthy computations. In general, just the very basic definitions of smooth manifolds, vector bundles, Banach spaces, etc, are needed to comprehend this theory (also see Appendix A for a guide to the mathematical background.) The only place where the treatment is mildly more technical is in Sec. V-C when the bundle homomorphism is constructed using partition of unity technique as a detailed computational application of the superspace theory.

\section{Overview of Electromagnetic Nonlocal MATERIALS}

\section{A. The Generic Nonlocal Response Model in Inhomogeneous Media}

In order to introduce the concept of nonlocality in the simplest way possible, let us first start with a scalar field theory setting. As mentioned in the introduction, vacuum classical fields cannot exhibit nonlocality, so in order to attain this phenomenon, one must consider fields in specialized domains. We then begin by reviewing the broad theory of such media. The goal is to outline the main ingredients of the spacetime configuration space on which such theories are often formulated in literature. To further simplify the presentation, we work in the regime of linear response theory: all media are assumed to be linear with respect to field excitation. If the medium response is described by the function $R(\mathbf{r}, t)$, while the exciting field is $F(\mathbf{r}, t)$, then the most general response is given by an operator equation of the form [29]

$$
R(\mathbf{r}, t)=\mathcal{L}\{F(\mathbf{r}, t)\},
$$


where $\mathcal{L}$ is a the linear operator describing the medium, and is ultimately determined by the laws of physics relevant to the structure under consideration [117]-[119]. Now, the entire physical process will occur in a spacetime domain. In nonrelativistic applications (like this paper), we intentionally separate and distinguish space from time. Therefore, let us consider a process of field-matter interaction where $t \in \mathbb{R}$, while we spatially restrict to a "small" region $\mathbf{r} \in D \subset \mathbb{R}^{3}$, where $D$ is an open set containing $\mathbf{r}$. ${ }^{6}$ Since the operator $\mathcal{L}$ is linear, one may argue (informally) that its associated Green's function $K\left(\mathbf{r}, \mathbf{r}^{\prime} ; t, t^{\prime}\right)$ must exist. Strictly speaking, this is not correct in general and one needs to prove the existence of the Green's function for every given linear operator on a case by case basis by actually constructing one [16], [120]. ${ }^{7}$ However, we will follow (for now) the common trend in physics and engineering by assuming that linearity alone is enough to justify the construction of the Green's function. If this is accepted, then we can immediately infer from the very definition of the Green's function itself that [1], [121]

$$
R(\mathbf{r}, t)=\int_{D} \int_{\mathbb{R}} \mathrm{d}^{3} r^{\prime} \mathrm{d} t^{\prime} K\left(\mathbf{r}, \mathbf{r}^{\prime} ; t, t^{\prime}\right) F\left(\mathbf{r}^{\prime}, t^{\prime}\right) .
$$

The relation (2) represents the most general response function of a (scalar) material medium valid for linear field-matter interaction regimes [45], [48]. The kernel (Green's) function $K\left(\mathbf{r}, \mathbf{r}^{\prime} ; t, t^{\prime}\right)$ is often called the medium response function. If we further assume that all of the material constituents of the medium are time-invariant (the medium is not changing with time), then the relation (2) maybe replaced by

$$
R(\mathbf{r}, t)=\int_{D} \int_{\mathbb{R}} \mathrm{d}^{3} r^{\prime} \mathrm{d} t^{\prime} K\left(\mathbf{r}, \mathbf{r}^{\prime} ; t-t^{\prime}\right) F\left(\mathbf{r}^{\prime}, t^{\prime}\right),
$$

where the only difference is that the kernel function's temporal dependence is replaced by $t-t^{\prime}$ instead of two separated arguments. Such superficially small difference has nevertheless considerable consequences. Most importantly, working with (3) instead of (2), it becomes possible to apply the Fourier transform in time to simplify the formulation of the problem. Indeed, taking the temporal Fourier transform of both sides of (3) leads to

$$
R(\mathbf{r}, \omega)=\int_{D} \mathrm{~d}^{3} r^{\prime} K\left(\mathbf{r}, \mathbf{r}^{\prime} ; \omega\right) F\left(\mathbf{r}^{\prime} ; \omega\right),
$$

where the Fourier spectra of the fields are defined by

$$
F(\mathbf{r} ; \omega):=\int_{\mathbb{R}} \mathrm{d} t F(\mathbf{r} ; t) e^{-\mathrm{i} \omega t}, R(\mathbf{r} ; \omega):=\int_{\mathbb{R}} \mathrm{d} t R(\mathbf{r} ; t) e^{-\mathrm{i} \omega t} .
$$

On the other hand, the medium response function's Fourier transform is given by the essentially equivalent formula

$$
K\left(\mathbf{r}, \mathbf{r}^{\prime} ; \omega\right):=\int_{\mathbb{R}} \mathrm{d}\left(t-t^{\prime}\right) K\left(\mathbf{r}, \mathbf{r}^{\prime} ; t-t^{\prime}\right) e^{-\mathrm{i} \omega\left(t-t^{\prime}\right)} .
$$

In this paper, we focus on time-invariant material media and hence work exclusively with frequency-domain expressions like (4).

\footnotetext{
${ }^{6}$ We assume the normal Euclidean topology on $\mathbb{R}^{3}$ for all spatial domains.

${ }^{7}$ This is argued in details in [121]. In particular, the recently-introduced current Green's function of electromagnetic devices was inspired by finding a Green's function structure similar to that corresponding to nonlocal media [122]-[124]
}

The generalization to the 3-dimensional (full-wave) electromagnetic picture is straightforward when the dyadic formalism is employed. The relation corresponding to (2) is

$$
\mathbf{R}(\mathbf{r}, t)=\int_{D} \mathrm{~d}^{3} r^{\prime} \int_{\mathbb{R}} \mathrm{d} t^{\prime} \overline{\mathbf{K}}\left(\mathbf{r}, \mathbf{r}^{\prime} ; t-t^{\prime}\right) \cdot \mathbf{F}\left(\mathbf{r}^{\prime}, t^{\prime}\right),
$$

where we have replaced the scalar fields $F(\mathbf{r})$ and $R(\mathbf{r})$ by vectors $\mathbf{F}(\mathbf{r}), \mathbf{R}(\mathbf{r}) \in \mathbb{R}^{3}$. The kernel function $K$, however, must be transformed into a dyadic function (tensor of second rank) $\overline{\mathbf{K}}\left(\mathbf{r}, \mathbf{r}^{\prime} ; t-t^{\prime}\right)$ [2], [118], [125]. In the (temporal) Fourier domain, (7) becomes

$$
\mathbf{R}(\mathbf{r}, \omega)=\int_{D} \mathrm{~d}^{3} r^{\prime} \overline{\mathbf{K}}\left(\mathbf{r}, \mathbf{r}^{\prime} ; \omega\right) \cdot \mathbf{F}\left(\mathbf{r}^{\prime} ; \omega\right)
$$

where

$$
\begin{gathered}
\overline{\mathbf{K}}\left(\mathbf{r}, \mathbf{r}^{\prime} ; \omega\right):=\int_{\mathbb{R}} \mathrm{d}\left(t-t^{\prime}\right) \overline{\mathbf{K}}\left(\mathbf{r}, \mathbf{r}^{\prime} ; t-t^{\prime}\right) e^{-\mathrm{i} \omega\left(t-t^{\prime}\right)}, \\
\mathbf{F}(\mathbf{r} ; \omega):=\int_{\mathbb{R}} \mathrm{d} t \mathbf{F}(\mathbf{r} ; t) e^{-\mathrm{i} \omega t}, \mathbf{R}(\mathbf{r} ; \omega):=\int_{\mathbb{R}} \mathrm{d} t \mathbf{R}(\mathbf{r} ; t) e^{-\mathrm{i} \omega t} .
\end{gathered}
$$

The essence of electromagnetic nonlocality can be neatly captured by the mathematical structure of the basic relation (7). In words, it says that the field response $\mathbf{R}(\mathbf{r})$ is determined not only by the excitation field $\mathbf{F}\left(\mathbf{r}^{\prime}\right)$ applied at location $\mathbf{r}^{\prime}$, but at all points $\mathbf{r}^{\prime} \in D$. Consequently, knowledge of the response at one point requires knowledge of the cause (excitation field) at an entire topologically local set $D$. On the other hand, if the medium is local, then the material response function can be written as

$$
\overline{\mathbf{K}}\left(\mathbf{r}, \mathbf{r}^{\prime} ; \omega\right)=\overline{\mathbf{K}}_{0}(\omega) \delta\left(\mathbf{r}-\mathbf{r}^{\prime}\right),
$$

where $\overline{\mathbf{K}}_{0}$ is a spatially constant tensor and $\delta\left(\mathbf{r}-\mathbf{r}^{\prime}\right)$ is the 3-dimensional Dirac delta function. In this case, (8) reduces to $[126]$

$$
\mathbf{R}(\mathbf{r} ; \omega)=\overline{\mathbf{K}}_{0}(\omega) \cdot \mathbf{F}(\mathbf{r} ; \omega)
$$

which is the standard constitutive relation of linear electromagnetic materials. Clearly, (12) says that only the exciting field $\mathbf{F}(\mathbf{r})$ data at $\mathbf{r}$ is needed to induce a response at the same location. In a nutshell, locality implies that the natural configuration space of the electromagnetic problem is just the point-like spacetime manifold $D \subset \mathbb{R}^{3}$ or the entire Euclidean space $\mathbb{R}^{3}$.

Remark III.1. One may attach the "infinitesimally immediate neighborhood" to a given point $\mathbf{r}$ where a response is sought. Indeed, according to (12), while only the exciting field at $\mathbf{r}$ is needed to compute the response, Maxwell's equations still need to be coupled with that local constitutive relation. The fact that these equations are differential equations implies that the "largest" domain beside the point $\mathbf{r}$ needed to carry over the mathematical description of the field-matter interaction physics is just the region infinitesimally close to $\mathbf{r}$.

Conventional boundary-value problems in applied electromagnetism are formulated in this manner, i.e., with a 3differential manifolds as the main problem space on which spatial fields live [118], [119], [121], [125], [127]-[131]. Note that strictly speaking, the full configuration space in 
local electromagnetism (also called normal optics) is the 4dimensional manifolds $D \times \mathbb{R}$ or $\mathbb{R}^{4}$ since either time $t$ or the (temporal) circular frequency $\omega$ must be included to engender a full description of electromagnetic fields. However, nonlocal materials are most fundamentally a spatial type of materials/metamaterials where it is the spatial structure of the field what carries most of the physics involved [121], [132]. For that reason, throughout this paper we investigate the required configuration spaces with focus mainly on the spatial degrees of freedom. This will naturally lead to the discovery of the fiber-bundle structure of nonlocality, the main topic of the present work.

\section{B. Spatial Dispersion in Homogeneous Nonlocal Material Domains}

Spatial dispersion is considered by some researchers as one of the most promising routes toward nonlocal metamaterials, e.g., see [4], [132]-[134]. It is by large the most intensely investigated class of nonlocal media, receiving both theoretical and experimental treatments by various research groups since the early $1960 \mathrm{~s} .{ }^{8}$ The basic idea is to restrict electromagnetism to the special but important case of media possessing translational symmetry, an important case attained when the medium is homogeneous. In such situation, the material tensor function satisfies

$$
\overline{\mathbf{K}}\left(\mathbf{r}, \mathbf{r}^{\prime} ; \omega\right)=\overline{\mathbf{K}}\left(\mathbf{r}-\mathbf{r}^{\prime} ; \omega\right) .
$$

The spatial Fourier transforms are defined by

$$
\overline{\mathbf{K}}(\mathbf{k}, \omega):=\int_{\mathbb{R}^{3}} \mathrm{~d}^{3}\left(r-r^{\prime}\right) \overline{\mathbf{K}}\left(\mathbf{r}-\mathbf{r}^{\prime} ; \omega\right) e^{i \mathbf{k} \cdot\left(\mathbf{r}-\mathbf{r}^{\prime}\right)},
$$

with

$$
\begin{aligned}
\mathbf{F}(\mathbf{k}, \omega) & :=\int_{\mathbb{R}^{3}} \mathrm{~d}^{3} r \mathbf{F}(\mathbf{r} ; t) e^{\mathrm{i} \mathbf{k} \cdot \mathbf{r}}, \\
\mathbf{R}(\mathbf{k}, \omega) & :=\int_{\mathbb{R}^{3}} \mathrm{~d}^{3} r \mathbf{R}(\mathbf{r} ; t) e^{\mathrm{ik} \cdot \mathbf{r}} .
\end{aligned}
$$

After inserting (13) into (8) and taking the spatial (3dimensional) Fourier transform of both sides, the following relation is obtained

$$
\mathbf{R}(\mathbf{k}, \omega)=\overline{\mathbf{K}}(\mathbf{k}, \omega) \cdot \mathbf{F}(\mathbf{k}, \omega) .
$$

The dependence of $\overline{\mathbf{K}}(\mathbf{k}, \omega)$ on the wavevector ("spatial frequency") $\mathbf{k}$ in addition to the the temporal frequency $\omega$ is the signature of spatial dispersion. As a spectral transfer function of the medium, $\overline{\mathbf{K}}(\mathbf{k}, \omega)$ includes all the information needed to obtain the nonlocal material domain's response to arbitrary spacetime field excitation functions $\mathbf{F}(\mathbf{r}, t)$ through the application of inverse 4-dimensional Fourier transform [4].

Remark III.2. In many treatments of the subject, the excitation field is taken as the electric field $\mathbf{E}(\mathbf{r}, t)$, while the response function is $\mathbf{D}(\mathbf{r}, t)$. In such formulation, the material tensor function $\overline{\mathbf{K}}(\mathbf{k}, \omega)$ takes into account both electric and magnetic effects [2]-[4], [22], [45], [46], [48], [135], [46], [47], [49], [136]. This is different from the permitivity tensor often invoked in local electromagnetism, which is based on

${ }^{8}$ See Sec. II-A and Remark III.3 for literature review. the popular multipole model of material media. A comparison between the two material response formalisms, the one based on $\overline{\mathbf{K}}(\mathbf{k}, \omega)$ and the multipole model is given in [47], [121], [132].

Remark III.3. (Historical Digression) Historically, spatial dispersion had been under the radar since the 1950s, especially in connection with researches on the optical spectra of material domains [137]-[139]. However, the first systematic and thorough treatment of the subject appeared in 1960s, especially in the first edition of Ginzburg book on plasma physics, which was dedicated to electromagnetic wave propagation in plasma media. The second edition of the book, published in 1970, contained a considerably extended treatment of the various mathematical and physical aspects of the electromagnetism of spatially dispersive media [2]. Spatial dispersion in crystals had been also investigated by Ginzburg and his coworkers during roughly the same time [140]-[142]. The book [20] contains good summaries on spatial dispersion research up to the end of the 1980s. More recently, media obtained by homogenizing arrays of wires, already very popular because of their connection with traditional (temporal) metamaterials, are known to exhibit spatial dispersion effects, though many researchers ignore that effect to focus on temporal dispersion [143]-[145]. Other types of periodic or large finite arrays of composed of unit cells like spheres and desks also exhibit spatial dispersion effects [146]. Nonlinear materials with observable nonlocality have also been investigated in the optical regime [147]. More recently, much of the reemergence of interest in spatial dispersion stems from the observation that the phenomenon cannot be ignored at the nanoscale [148], especially those of low-dimensional structures like carbon nanotubes [51], [54], [149] and graphene [150], [151]. The subject was also introduced at a pedagogical level for applications involving current flow in spatially dispersive conductive materials like plasma and nanowires [152].

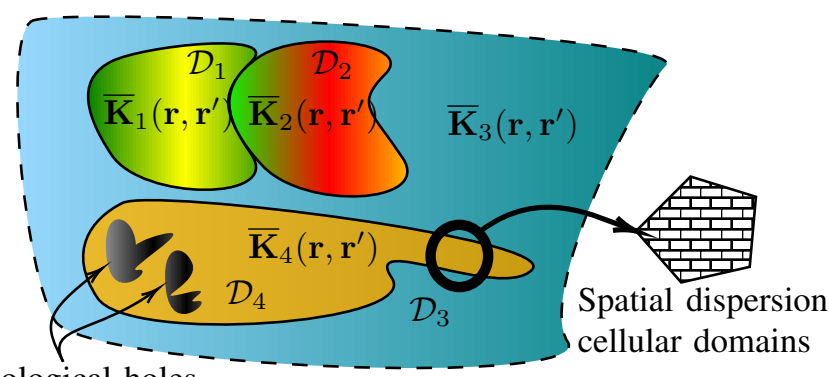

Topological holes

Fig. 1: A generic depiction of an electromagnetic nonlocal metamaterial system. Each of the domains $\mathcal{D}_{n}$ is captured by a general linear nonlocal response function $\overline{\mathbf{K}}_{n}\left(\mathbf{r}, \mathbf{r}^{\prime}\right)$.

Complex heterogeneous arrangements of various nonlocal materials can be realized by juxtaposing several subdomains where each is homogeneous, hence, can be described by a spatial dispersion profile $\overline{\mathbf{K}}(\mathbf{k}, \omega)$. The idea is that even materials inhomogeneous at a given spatial scale tend to become homogeneous at a finer spatial level, leading to a "grid-like" spatially dispersive cellular building blocks at the 
lower level. In Fig. 1, we show a nonlocal metamaterial system with various multiscale structures. A large nonlocal domain, e.g., $\overline{\mathbf{K}}_{3}\left(\mathbf{r}, \mathbf{r}^{\prime}\right)$ in the figure, acts like a "substrate" holding together several other smaller material constituents, such as $\overline{\mathbf{K}}_{n}\left(\mathbf{r}, \mathbf{r}^{\prime}\right), n=1,2,4$. We envision that each nonlocal subdomain may possess its own especially tailored nonlocal response function profile serving one or several applications. ${ }^{9}$ By concatenating multiple regions, interfaces between subdomains with different material constitutive relations are created. We show here subdomains $\mathcal{D}_{n}, n=1,2,3,4$, and the possible material interfaces include $\mathcal{D}_{1} / \mathcal{D}_{2}, \mathcal{D}_{1} / \mathcal{D}_{3}, \mathcal{D}_{2} / \mathcal{D}_{3}, \mathcal{D}_{3} / \mathcal{D}_{4}$. Recall that in local electromagnetism each material interface should be assigned a special electromagnetic boundary condition in order to ensure the existence of a unique solution to the problem [125]. This however is not possible in nonlocal electromagnetism.

Indeed, and as already mentioned earlier, nonlocal electromagnetism introduces several subtle issues that are absent in the local case: additional boundary conditions are often invoked to handle the transition of fields along barriers separating different domains, like between two nonlocal domains or even one nonlocal and another local domain [4], [44], [153]. The topological fiber bundle theory to be developed in Sec. $\mathrm{V}$ will provide a clarification of why this is so since it turns out the traditional spacetime approach often employed in local electromagnetism is not necessarily the most natural one. There is a need to examine in more details the existence of multiple topological scales in nonlocal metamaterials, and this paper will provide some new insights into such issues.

For completeness, we note below three directly observable topological scales that do not require the more elaborate mathematical apparatus to be detailed in later Sections. We list these as follows:

1) The first is the already stated separation between different nonlocal domains like $\mathcal{D}_{1}$ and $\mathcal{D}_{2}$.

2) The second is the case captured by the inset in the right hand side in Fig. 1. Fine "microscopic" cells, each homogeneous and hence describable by a response function of the form $\overline{\mathbf{K}}(\mathbf{k}, \omega)$, can be combined to build up a complex effective nonlocal response tensor $\overline{\mathbf{K}}_{n}\left(\mathbf{r}, \mathbf{r}^{\prime}\right)$ over its topologically global domain $\mathcal{D}_{n}$. Such juxtaposition at the microscopically local level leading to global behaviour is a classic example of multiscale physics, but here it acquires even higher importance since both the constituent cells (rectangular "bricks" in the inset of Fig. 1) and the global domain level $\mathcal{D}_{n}$ are already electromagnetically nonlocal.

3) Finally, the third directly observable topological scale is that connected to what we termed "topological holes" in Fig. 1. These are arbitrarily-shaped gaps, like holes, vias, etchings, etc, that are intentionally introduced in order to influence the the electromagnetic response by modifying the topology of the 3-dimensional material manifolds $\mathcal{D}_{n}$.

\footnotetext{
${ }^{9} \mathrm{~A}$ discussion of some possible engineering applications is provided in Sec. IX-B.
}

Remark III.4. (Distinction Between Electromagnetic and Topological Locality/Nonlocality) The terms local and global possess two different senses, one electromagnetic, the other spatio-geometric. Elucidating this subtle interconnection between the two senses will be one of the main objectives of the present work but we will first need to introduce the various relevant micro-scale topological concepts to be given below (see also Remark VII.1.)

\section{The Main Topological Structure of Nonlocal ELECTROMAGNeTIC DOMAINS}

\section{A. Introduction}

Let the nonlocality domain of the electromagnetic medium, the region $D \subset \mathbb{R}^{3}$ in (8), be bounded. Corresponding to (1), a similar operator equation in the frequency domain can be assumed to represent the most general form of a nonlocal electromagnetic medium, namely

$$
\mathbf{R}(\mathbf{r} ; \omega)=\mathcal{L}_{\omega}\{\mathbf{F}(\mathbf{r} ; \omega)\},
$$

where the nonlocal medium linear operator is itself a function of frequency. For simplicity, when it is understood from the context that the material response operator is formulated in the frequency domain, the dependence on $\omega$ in its expression will be removed.

We are going to propose a change in the mathematical framework inside which electromagnetic nonlocality is usually defined. This will be done in two stages:

- Initially, in the present Section, we introduce the rudiments of the main physics-based micro-topological structure associated with EM nonlocality without going into considerable mathematical details. The aim is to familiarize ourselves with the minimal necessary physical setting and how it naturally gives rise to a finer picture of the material domain compared with the traditional (and much simpler) topological structure of local electromagnetism based on spacetime points.

- In the second stage, covered in Sec. V, a more careful mathematical picture is developed using the theory of topological fiber bundles. We eventually show (Sec. V-C) that the EM nonlocal operator (17) can be reformulated as a Banach bundle map (homomorphism) over the 3dimensional space of the material domain under consideration. Computational examples and applications are provided in the later Sections.

The key conceptual idea behind the entire theory presented here is that of topological microdomains associated with the electromagnetism of nonlocal media, which we first develop thematically in the next Sec. IV-B before moving subsequently to the rigorous and exact topological formulation of Sec. V.

\section{B. The Concept of Topological Microdomains in Nonlocal electromagnetism}

In conventional frequency-domain local electromagnetism, the boundary-value problem of multiple domains is formulated as a set of coupled partial differential equations or integrodifferential equations interwoven with each other via the 
appropriate material interface boundary conditions dictating how fields change while crossing the various spatial regions inside which the equations hold [118], [125], [127]. This has been traditionally achieved by taking up the electromagnetic response function $\overline{\mathbf{K}}\left(\mathbf{r}, \mathbf{r}^{\prime} ; \omega\right)$ as an essential key ingredient of the problem description, which traditionally has been exploited in two stages: First, the constitutive relations enter into the governing equations in each separate solution domain. Second, the constitutive relations themselves are used in order to construct the proper electromagnetic boundary conditions prescribing the continuity/discontinuity behaviour of the sought field solutions as they move across the various interfaces separating domains with different material properties.

Unfortunately, it has been well known for a long time that it is not possible to formulate a universal electromagnetic boundary condition for nonlocal medium, especially for the case of spatial dispersion. This will be discussed later with more details in Sec. VIII, but also see the discussion around additional boundary conditions (ABC) in Sec. II-A. For now, we concentrate on gaining a deeper understanding of the structure of spatial nonlocality in electromagnetism.

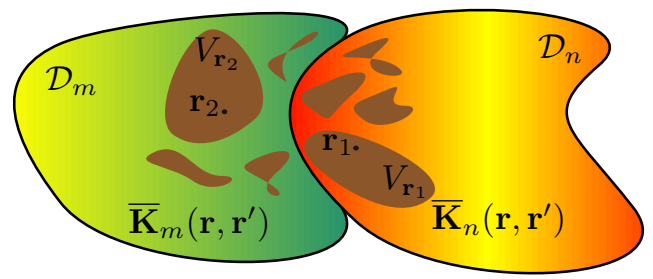

Fig. 2: The micro-topological structure of nonlocal metamaterial systems includes more than just the 3-dimensional spatial domains $\mathcal{D}_{n}, n=1,2, \ldots$ It is best captured by classes $\mathcal{V}\left(\mathcal{D}_{n}\right)$ composed of various open sets $V_{\mathbf{r}} \subset \mathcal{D}_{n}$ based at each point $\mathbf{r} \in \mathcal{D}_{n}$. On every such subset a vector field is defined, representing the EM excitation field. The collection of all vector fields on a given set $V_{\mathbf{r}}$ gives rise to a linear topological function space $\mathcal{F}\left(V_{\mathbf{r}}\right)$. The topologies of the base spaces $\mathcal{D}_{n}$, the nonlocal micro-domains $V_{\mathbf{r}}$, and the function spaces $\mathcal{F}\left(V_{\mathbf{r}}\right)$ collectively give rise to a total "macroscopic" topological structure (superspace) considerably more complex than the base spaces $\mathcal{D}_{n}$.

A key starting observation is how nonlocality forces us to associate with every spacetime point $(\mathbf{r}, t)$ - or frequencyspace point $(\mathbf{r} ; \omega)$ - a topological neighborhood of $\mathbf{r}$, say $V_{\mathbf{r}}$, such that $\mathbf{r} \in V_{\mathbf{r}}$. For now, let us assume that the spatial material domain $D \ni \mathbf{r}$ is just an open set in the technical sense of the topology of the Euclidean space $\mathbb{R}^{3}$ inherited from the standard Euclidean metric [154]. By restricting $D$ to be open, we avoid the notorious problem of dealing with boundaries or interfaces between such (possibly overlapping) open sets. That is, the topological closure of $D$, denoted by $\operatorname{cl}(D)$, is excluded from the domain of nonlocality. Let $D$ be the maximal such topological neighbored for the problem under consideration. ${ }^{10}$ We now associate with each point $\mathbf{r}$ a smaller open set $V_{\mathbf{r}}$ such that $\mathbf{r} \in V_{\mathbf{r}}, V_{\mathbf{r}} \subset D$ for all $\mathbf{r} \in D$. (The fact that $D$ is assumed open makes this possible.)

Now, instead of considering fields like $\mathbf{R}(\mathbf{r})$ and $\mathbf{F}(\mathbf{r})$ defined on the entire maximal domain of nonlocality $D$ (which can grow "very large,") we propose to reformulate the problem of nonlocal electromagnetic materials in a topologically local form by noting that the physics of field-matter interactions gives the EM response at location $\mathbf{r}$ due to excitation fields essentially confined within a "smaller domain" around $\mathbf{r}$, namely the open set $V_{\mathbf{r}}{ }^{11}$ On the other hand, if the response at another different point $\mathbf{r} \neq \mathbf{r}^{\prime}$ is needed, then a new - generally different - small open set $V_{\mathbf{r}^{\prime}}$ will be required. That is, in general we allow that $V_{\mathbf{r}} \neq V_{\mathbf{r}^{\prime}}$, even though we expect that typically there is some overlap between these two small local domains of electromagnetic nonlocality, i.e., $V_{\mathbf{r}} \cap V_{\mathbf{r}^{\prime}} \neq \varnothing$, especially if $\left|\mathbf{r}-\mathbf{r}^{\prime}\right|$ is small.

The "smaller sets" $V_{\mathbf{r}}, \mathbf{r} \in D$, will be dubbed nonlocal microdomains or just microdomains in short. A possible definition is the following:

Definition IV.1. (EM Nonlocality Microdomains) Consider a material domain $D$ with the associated EM response function $\overline{\mathbf{K}}\left(\mathbf{r}^{\prime}, \mathbf{r}\right)$. We define the EM nonlocality microdomain $V_{\mathbf{r}} \subset D$ based at $\mathbf{r} \in D$ as the interior of the compact support of $\overline{\mathbf{K}}\left(\mathbf{r}^{\prime}, \mathbf{r}\right)$. The support is defined by $\operatorname{supp} \overline{\mathbf{K}}\left(\mathbf{r}^{\prime}, \mathbf{r}\right):=\left\{\mathbf{r}^{\prime} \in\right.$ $\left.D,\left\|\overline{\mathbf{K}}\left(\mathbf{r}^{\prime}, \mathbf{r}\right)\right\| \neq 0\right\}$, where $\|\cdot\|$ is a suitable tensor norm, for example the matrix norm.

Remark IV.1. It can be shown that he collection of open sets $\left\{V_{\mathbf{r}}, \mathbf{r} \in D\right\}$ induces a topology on the total space occupied by the nonlocal material. This topology will be referred to in what follows by the term microdomains topology. In local media, the microdomains topology reduces to the trivial discrete topology $\{\{\mathbf{r}\}, \mathbf{r} \in D\}$ since the external field interacts only with the point $\mathbf{r}$ at which it is applied and hence $V_{\mathbf{r}}=\{\mathbf{r}\}$.

The set of EM nonlocality microdomains (microdomains for short) defined above explicate the fine micro-topological structure of nonlocal electromagnetic domains at a spatial scale different from that of the (topologically "larger") material domain $D$ itself and are fundamental for the theory developed in this paper.

\section{Construction of Excitation Field Functions Spaces on the Topological Microdomains of Nonlocal Media}

Next, after enriching the MTM domain $D$ with the finer topology of nonlocality microdomains $V_{\mathbf{r}}, \mathbf{r} \in D$, we will equip this total medium with additional mathematical structure based on the physics of field-matter interaction. Consider the set of all sufficiently differentiable vector fields $\mathbf{F}(\mathbf{r})$ defined on $V_{\mathbf{r}}, \mathbf{r} \in D$. This set possesses an obvious complex vector space structure: for any complex numbers $a_{1}, a_{2} \in \mathbb{C}$, the sum

$$
a_{1} \mathbf{F}_{1}\left(\mathbf{r}^{\prime}\right)+a_{2} \mathbf{F}_{2}\left(\mathbf{r}^{\prime}\right)
$$

\footnotetext{
${ }^{10}$ If $D$ asymptotically grows into an unbounded region, then the problem reduces to that of homogeneous unbounded domain (bulk media), well treated in the basic literature on spatial dispersion.

${ }^{11}$ This is well known from the quantum theory of nonlocality [4], [46], [48], but concrete examples illustrating this behaviour will be given in Sec. VII.
} 
is defined on $V_{\mathbf{r}}$ whenever $\mathbf{F}_{1}\left(\mathbf{r}^{\prime}\right)$ and $\mathbf{F}_{2}\left(\mathbf{r}^{\prime}\right)$ are, while the null field plays the role of the origin. In what follows, we will denote such function spaces by $\mathcal{F}\left(V_{\mathbf{r}}\right)$ or just $\mathcal{F}$ if it is understood from the context on which material spatial domains the fields are defined.

Remark IV.2. It is possible to equip $\mathcal{F}\left(V_{\mathbf{r}}\right)$ with a suitable topology in order to measure how "near" to each other are any two fields defined on $V_{\mathbf{r}}$, e.g., see [154]-[156]. Therefore, $\mathcal{F}\left(V_{\mathbf{r}}\right)$ becomes a topological vector space [154], in particular a Sobolev space, which is not only a Banach space (normed space), but also a Hilbert space (inner product space) [157][159].

\section{The Global Topological Structure of Nonlocal Electromag- netic Material Domains: First Look}

In light of the analysis above, each microdomain $V_{\mathbf{r}}$ induces an infinite-dimensional linear function space (Sobolev) $\mathcal{F}\left(V_{\mathbf{r}}\right)$ indexed by the position $\mathbf{r} \in D$, with topology essentially determined by the geometry of $V_{\mathbf{r}}$. On the other hand, this latter geometry is obtained from the physics of field-matter interaction in nonlocal media. Consequently, the physical content of nonlocal materials is encoded at the topologically micro-local level expressed by the following structure:

$$
D \ni \mathbf{r} \underset{\text { EM Nonlocality }}{\stackrel{\text { Physical Data }}{\longrightarrow}} V_{\mathbf{r}} \underset{\text { Sobolev Space }}{\stackrel{\text { Mathematical Data }}{\longrightarrow}} \mathcal{F}\left(V_{\mathbf{r}}\right) \text {. }
$$

If we denote the relevant collections of subsets as

$$
\mathcal{V}(D):=\left\{V_{\mathbf{r}} \subset D \mid \mathbf{r} \in D, V_{\mathbf{r}} \text { is open }\right\},
$$

$\mathcal{G}[\mathcal{V}(D)]:=\left\{\mathcal{F}\left(V_{\mathbf{r}}\right) \mid \mathbf{r} \in D, \mathcal{F}\left(V_{\mathbf{r}}\right):\right.$ Sobolev function space $\}$,

then (18) can be neatly captured by the ordered triplet

$$
D \times \mathcal{V}(D) \times \mathcal{G}[\mathcal{V}(D)] .
$$

Let us unpack this compact structure step by step as follows:

1) Each open domain in $D \subseteq \mathbb{R}^{3}$ will by assigned a distribution $\mathcal{V}(D)$ of open sets $V_{\mathbf{r}}$, i.e., the EM nonlocality microdomains topology defined in Sec. IV-B. Physically, it expresses the fine micro-topological structure of electromagnetic nonlocality.

2) The structure $\mathcal{V}(D)$ is solely determined by the fieldmatter interaction physics. A concrete example will be given in Sec. VII.

3) We further emphasize that the various sets $V_{\mathbf{r}} \in \mathcal{V}(D)$ constitute an open cover of $D$, that is, we have

$$
D=\bigcup_{\mathbf{r} \in D} V_{\mathbf{r}}
$$

In this way, the model can accommodate excitation fields $\mathbf{F}(\mathbf{r})$ applied at every point in $\mathbf{r} \in D$.

4) The decomposition of the material domain $D$ into smaller building blocks exemplified by (22) is fundamental for computational topological models of nonlocal MTMs. For example, in Sec. VII we will use this expansion to construct a topological coarse-grained model for inhomogeneous nonlocal semiconductor metamaterials.
5) Finally, the topology $\mathcal{V}(D)$ induces the space $\mathcal{G}[\mathcal{V}(D)]$ of function spaces $\mathcal{F}\left(V_{\mathbf{r}}\right), \mathbf{r} \in D$, where each vector field is defined on one element $V_{\mathbf{r}}$ chosen from the topology $\mathcal{V}(D)$.

It is interesting to note how within the framework proposed above a kind of constructive "division of labour" is shared between physics and mathematics in order to generate the various required multiscale structures characteristic of electromagnetic nonlocality. This is also the source of some potential difficulties hidden in the topological structure (21). Indeed, we will next try to smooth out the differences between the two main substructures $\mathcal{V}(D)$, controlled mostly by physics, on one side, and $\mathcal{G}[\mathcal{V}(D)]$, which is dominated by mathematical considerations. One way to achieve this is by unifying the entire total topological structure (21) that can encode all of the substrucutres of (21) within a single rich enough "metastructure" : the Banach vector bundle superspace (Sec. V).

\section{E. A Reformulation of the Electromagnetic Response Function}

It is now possible to provisionally construct the EM response function by working on the fundamental topological domain structure (21) instead of the global domain $D$, the later being the favored arena of conventional electromagnetic theory that we would like to move beyond in this paper. The response function $\mathbf{R}(\mathbf{r})$ will be re-expressed by the map

$$
\mathbf{R}: D \times \mathcal{V}(D) \rightarrow \mathbb{C}^{3},
$$

where the codomain is taken to be $\mathbb{C}^{3}$ because the electric or magnetic response functions $\mathbf{D}$ or $\mathbf{B}$, respectively, are complex vector fields in the frequency domain. ${ }^{12}$ The value of the EM nonlocal response field due to excitation field $\mathbf{F}(\mathbf{r})$ applied at a microdomain $V_{\mathbf{r}}$ can be computed by means of

$$
\mathbf{R}(\mathbf{r} ; \omega)=\int_{V_{\mathbf{r}}} \mathrm{d}^{3} r^{\prime} \overline{\mathbf{K}}\left(\mathbf{r}, \mathbf{r}^{\prime} ; \omega\right) \cdot \mathbf{F}\left(\mathbf{r}^{\prime} ; \omega\right) .
$$

Although (24) may appear at first sight to be only slightly different from (8), the underlying difference between the two formulas is significant. In essence, the construction of the EM response field $\mathbf{R}(\mathbf{r})$ via the map (23) amounts to topological localization of electromagnetic nonlocality since in the latter case the EM response function $\overline{\mathbf{K}}\left(\mathbf{r}, \mathbf{r}^{\prime} ; \omega\right)$ is no longer allowed to extend globally onto "large and complicated material domains." Indeed, with the recipe (24) only the response to "small" - or more rigorously topologically local - domains, namely the microdomains $V_{\mathbf{r}}$, is admitted. On the other hand, in order to find the response field $\mathbf{R}(\mathbf{r})$ everywhere in $D$, one needs to use sophisticated topological techniques to extend the response from one point to another till it covers the entirety of $D$. This local-to-global extension application of differential topology is discussed in details in Sec. V-C and again briefly in Sec. IX-A.

\footnotetext{
${ }^{12}$ In this section and the one to follow, we don't worry much about the details of the electromagnetic model and for simplicity assume that only one vector field $\mathbf{F}$ acts as excitation and one response field $\mathbf{R}$ is induced. More complex media like bi-anisotropic domains and others [160] may also be treated within this formulation. For example, if two response field are needed, the codomain in (23) can be simply changed to $\mathbb{C}^{6}$.
} 
In this manner, it becomes possible to provide an alternative, more detailed explication of the behaviour of the medium at topological interfaces (boundary conditions in nonlocal metamaterials are treated - provisionally - in Sec. VIII) and also explore the effect of the topology of the bulk medium itself on the allowable response functions and the production of non-trivial edge state, with obvious applications to nonlocal metamaterials (see the discussion of nonlocal and topological metamaterials applications in Sec. IX-B.)

\section{The Fiber-Bundle Superspace Formalism in the Electromagnetism of Generic Nonlocal Media}

Here, an outline of the direct construction of a fiber (Banach) bundle over an entire (global) nonlocal generic material domain is given, where our purpose is to build into every point $\mathbf{r} \in U_{i}$ a fiber superspace $\mathcal{F}_{i}$. The contents of this section are the most technically advanced in this paper. Readers interested in applications may skim through Secs. V-A and V-B, skip Sec. $\mathrm{V}-\mathrm{C}$, then move directly to Sec. VI for a general summary of the fiber bundle algorithm. Concrete computational models are outlined in Sec. VII using a practical nonlocal model, while additional remarks and discussions about current and future uses of the theory are provided in Sec. IX. However, even readers not fully familiar with differential manifold theory will benefit from reading the present technical Section because we strive to illustrate the physical intuition behind the various mathematical computations and steps therein.

\section{A. Preparatory Step: Promoting the Material Domain D to a Manifold $\mathcal{D}$}

In order to investigate in depth the fundamental physicomathematical constraints imposed on EM nonlocal metamaterials, the material domain $D$ considered so far should be promoted to a differential manifold structure [19], [155], [156], [161], [162]. There are several reasons why this is highly desirable:

1) It provides a natural and obvious generalization of the basic structure (21) from the mathematical perspective.

2) Engineers often need to insert metamaterials into specific device settings, hence the shape of the material becomes highly restricted. It is therefore important to develop efficient tools to deal with variations of geometric and topological degrees of freedom and how they could possibly impact the design process.

3) Applied scientists and engineers are often interested in deriving fundamental limitation on metamaterials, e.g., What are the ultimate allowable response-excitation relations or constitutive response functions possible given this material domain topology or that?

4) Sophisticated full-wave electromagnetic numerical solvers prefer working with local coordinates in order to handle complicated shapes, even if a global coordinate system is sometimes available, making the deployment of 3-manifold structures for describing the material domain $D$ useful.

5) In topological photonics and materials [111], most applications seem to focus on lower-dimensional states of matter like those associated with quantum Hall effects and edge states (surface waves). There, new phenomena appear at materials where the base space (material domain $D$ ) is a 2 -surface, which is best described mathematically as a differential 2-manifold.

For all these reasons, it is expedient to ascribe to the domain $D$ the most general mathematical expression possible, which in our case amounts to equipping the material/metamaterial's spatial domain with a smooth manifold structure. If we denote by $\mathcal{D}$ this 3 -manifold, then, being a subset of $\mathbb{R}^{3}$, there is a natural differential structure defined on it, that inherited from the 3-dimensional Euclidean space itself. This differential 3-manifold structure will be presupposed in the remaining parts of this paper. Following the standard theory of smooth manifolds, let $\left(U_{i}, \phi_{i}\right)$ be a collection of charts (an atlas), labeled by $i \in I$, an index set, which equips $\mathcal{D} \subset \mathbb{R}^{3}$ with a differential 3-manifold structure. ${ }^{13}$ This constitutes the differential atlas on $\mathcal{D}$ which will be used in what follows.

\section{B. Attaching Fibers to Generic Points in the Nonlocal Mate- rial Manifold D}

Our current goal is to attach a vector fiber (a linear function space in this case) at every point $\mathbf{r} \in \mathcal{D}$, namely the function space $\mathcal{F}\left(V_{\mathbf{r}}\right)$ introduced in Sec. IV-C. It turns out that accomplishing this requires finding suitable "compatibility laws" dictating how coordinates change when two intersecting charts $U_{i}$ and $U_{j}$ interact with each other, which is typical in such types of constructions [19]. In particular, we will need later to find the law of mutual transformation of vectors in the fibers $\mathcal{F}\left(V_{\phi_{i}(\mathbf{r})}\right)$ and $\mathcal{F}\left(V_{\phi_{j}(\mathbf{r})}\right)$. Here, the expression $\mathcal{F}\left(V_{\phi_{i}(\mathbf{r})}\right)$ means the fiber superspace attached to the point whose coordinates are $\phi_{i}(\mathbf{r})$, i.e., the function space where all functions are expressed in terms of the language of the $i$ th chart $\left(U_{i}, \phi_{i}(\mathbf{r})\right)$.

In this connection, the major technical problem facing us is the following: Since the differential structure associated with charts $\left(U_{i}, \phi_{i}(\mathbf{r})\right)$ can be fixed by essentially mathematical considerations alone, while the collection of microdomains $\mathcal{V}\left(\mathcal{D}=\left\{V_{\mathbf{r}}, \mathbf{r} \in \mathcal{D}\right\}\right.$ is solely determined by the physics of electromagnetic nonlocality (Sec. IV), there is no direct and simple way to express the transformation of vectors in $\mathcal{F}\left(V_{\phi_{i}(\mathbf{r})}\right)$ into vectors in $\mathcal{F}\left(V_{\phi_{j}(\mathbf{r})}\right)$ because several different coordinate patches other than $U_{i}$ and $U_{j}$, belonging to the differential 3-manifold $\mathcal{D}$ atlas, might be involved in building up the microdomain $V_{\mathbf{r}}$.

The above technical problem will be solved in Sec. V-C by using the technique of partition of unity borrowed from differential topology [19], [155], [161]. It will allow us to split up each full microdomain $V_{\mathbf{r}}$ into several suitable submicrodomains (details below), which can be later be joined up together in order to give back the original EM nonlocality microdomain $V_{\mathbf{r}}$.

For now, we start by recalling that the microdomain structure represented by the collection $\mathcal{V}(\mathcal{D}):=\left\{V_{\mathbf{r}}, \mathbf{r} \in \mathcal{D}\right\}$ is an open cover of the manifold $\mathcal{D}$. Therefore, and since the

\footnotetext{
${ }^{13}$ For simplicity, we will refer to the points of the manifold $\mathcal{D}$ by $\mathbf{r}$, i.e., using the language of the global (ambient) Euclidean space $\mathbb{R}^{3}$.
} 
material domain manifold $\mathcal{D}$ possesses a countable topological base [154], it contains a locally finite open cover subordinated to $\mathcal{V}(\mathcal{D})[19],[161] .^{14}$

Specifically, the theorem just mentioned implies that an atlas $\left(U_{i}, \phi_{i}\right), i \in I$, with diffeomorhisms

$$
\phi_{i}: U_{i} \rightarrow \mathbb{R}^{3},
$$

describing the differential structure of the manifold $\mathcal{D}$ exists such that the elements $\left\{U_{i}, i \in I\right\}$ constitute the above mentioned locally finite subcover subordinated to the microdomains collection $\mathcal{V}(\mathcal{D})$. Moreover, the images $\phi_{i}\left(U_{i}\right)$ are open balls centered around 0 in $\mathbb{R}^{3}$ with finite radius $a>0$ (henceforth, such balls will be denoted by $B_{a}$ ) [19].

In this way, the physics-based open cover set $\mathcal{V}(\mathcal{D})$ provides a first step toward the construction of a complete topological description of the electromagnetic nonlocal microdomain structure. The reason is that the coordinate patches $\left(U_{i}, \phi_{i}\right), i \in I$, are subordinated to the microdomains $\left\{V_{\mathbf{r}}, \mathbf{r} \in\right.$ D \} [19].

It is also known that there exists a partition of unity associated with the $\mathcal{D}$-atlas $\left(U_{i}, \phi_{i}\right), i \in I$, constructed above summarized by the following lemma [19], [155], [156], [161], [162]:

Lemma V.1. (Partition of Unity) There is a collection of functions

$$
\psi_{i}: U_{i} \subset \mathcal{D} \rightarrow \mathbb{R}
$$

satisfying the following requirements:

1) $\psi_{i}(\mathbf{r}) \geq 0$ and each function is $C^{p}, p \geq 1$. $^{15}$

2) The support of $\psi_{i}(\mathbf{r})$, denoted by $\operatorname{supp} \psi_{i}$, is contained within $U_{i}$, i.e., $\operatorname{supp} \psi_{i} \subset U_{i}{ }^{16}$

3) Since the open cover $U_{i}, i \in I$, is locally finite, at each point $\mathbf{r} \in \mathcal{D}$, only a finite number of $U_{i}$ will intersect $\mathbf{r}$.

4) Let the set of indices of those intersecting $U_{i} s$ be $I_{\mathbf{r}}$. Then we require that

$$
\sum_{i \in I_{\mathbf{r}}} \psi_{i}(\mathbf{r})=1
$$

where the sum is always convergent because the set $I_{\mathbf{r}}$ is finite.

Remark V.1. It can be shown that the sets $\phi_{i}^{-1}\left(B_{a / 3}\right), i \in$ $I$, already cover $\mathcal{D}$ [161]. The closure $\operatorname{cl}\left\{\phi_{i}^{-1}\left(B_{a / 3}\right)\right\}$ may be taken to constitute the support of $\psi_{i}(\mathbf{r})$, while $\psi_{i}(\mathbf{r})=0$ for all $\mathbf{r} \notin \operatorname{supp}\left\{\psi_{i}(\mathbf{r})\right\}$ [19], [161], [164]. The partition of unity functions $\psi_{i}$ can be computationally constructed using standard methods, most prominently the bump functions, e.g., see [161], [165].

The motivation behind the deployment of the partition of unity technique and how it immediately arises in connection

\footnotetext{
${ }^{14}$ See [154], [162] for the full technical definition of subordinated cover. A collection of subsets of a topological space is said to be locally finite, if each point in the space has a neighbourhood that intersects only finitely many of the sets in the collection. What we need here is that there exists some $i$ and $\mathbf{r}$ such that $U_{i}$ is inside $V_{\mathbf{r}}$, i.e., $U_{i} \subseteq V_{\mathbf{r}}$ where $\mathbf{r} \in U_{i}$.

${ }^{15}$ The function space $C^{p}$ is comprised of the set of real functions that are continuously differentiable $p$-times [155], [163].

${ }^{16}$ The support is defined as the (topological) closure of the set $\{\mathbf{r} \in$ $\left.\mathcal{D} \mid \psi_{i}(\mathbf{r}) \neq 0\right\}$ [16], [162], [163].
}

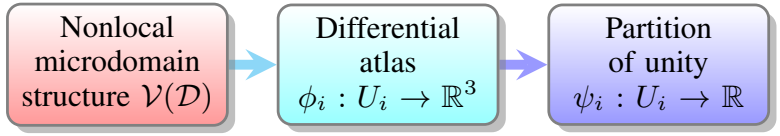

Fig. 3: The three-step process of constructing micro-coordinate representations of EM nonlocality starting from the nonlocal microdomain set and ending with the partition of unity on the MTM superspace.

with our fundamental EM nonlocal structure should now be clear. we have found that the following three-step process is natural:

1) Initially, the physics-based collection of sets $\mathcal{V}(\mathcal{D})=$ $\left\{V_{\mathbf{r}}, \mathbf{r} \in \mathcal{D}\right\}$, i.e., the EM nonlocal microdomain structure based on each point $\mathbf{r}$ in the nonlocal metamaterial $\mathcal{D}$, is obtained using a suitable physical microscopic theory or some other procedure. ${ }^{17}$

2) Introduce a differential atlas $\left(U_{i}, \phi_{i}(\mathbf{r})\right), i \in I$ on the smooth manifold $\mathcal{D}$ subordinated to $\mathcal{V}(\mathcal{D})$ and representing the nonlocal material domain under consideration.

3) Finally, the same atlas is linked to a set of functions $\psi_{i}(\mathbf{r})$ (partition of unity) that can be recruited as "topological bases" to expand any differentiable field excitation function into sum of individual sub-fields defined on open subsets of the material domain $\mathcal{D}$ (see Sec. V-C.)

The three-step process outlined above is summarized in Fig. 3 , illustrating how to progressively construct micro-coordinate systems allowing one to see through increasingly smaller spatial scales in the fundamental characterization of electromagnetic material nonlocality.

The key idea to be developed next is that both the base manifold $\mathcal{D}$ and the nonlocal EM microdomains $V_{\mathbf{r}}$ are described locally (in the topological sense) by the same collection of charts, namely $\left(U_{i}, \phi_{i}(\mathbf{r})\right), i \in I$. This will permit us to construct a direct unified description of both the base manifold $\mathcal{D}$ and its fibers, i.e., the linear topological function spaces $\mathcal{F}\left(V_{\mathbf{r}}\right)$, the latter being the model of the physical electromagnetic fields exciting the nonlocal material $\mathcal{D}$.

The construction of a fiber-bundle superspace for nonlocal electromagnetic materials will be completed in two steps:

- Step I: Construct a tailored fiber bundle based on the partition of unity charts $\left(U_{i}, \phi_{i}(\mathbf{r})\right)$ introduced above.

- Step II: The original physical structure (21) is recovered by gluing together various sub-microdomain $U_{i} \subseteq V_{\mathbf{r}}$ of each EM nonlocal microdomain $V_{\mathbf{r}}$.

We start with the Step I and leave the more complicated Step II to Section V-C.

Consider the $\left(U_{i}, \phi_{i}(\mathbf{r})\right), i \in I$, as our atlas on the 3manifold $\mathcal{D}$ introduced in Sec. V-A. At each point $\mathbf{r} \in U_{i}$, we attached a linear topological space $\mathcal{F}\left(U_{i}\right)$ defined as the Sobolev space $W^{p, 2}\left(U_{i}\right), p \geq 1$, of functions on the open set $U_{i}$, i.e., we write

$$
\mathcal{F}\left(U_{i}\right):=\left\{\psi_{i}(\mathbf{r}) \mathbf{F}(\mathbf{r}), \mathbf{r} \in U_{i} \mid \text { Sobolev Space } W^{p, 2}\left(U_{i}\right)\right\},
$$

\footnotetext{
${ }^{17}$ See Sec. VII for one possible method and examples.
} 
where $\mathbf{F}(\mathbf{r})$ is a suitable $C^{p, 2}$ vector field.

Remark V.2. For the precise technical definition of the infinite-dimensional Sobolev function space $W^{p, 2}\left(U_{i}\right)$, see [157], [158]. Appendix A provides some additional information on the literature. Sec. IV-C gives a simplified intuitive definition of the physics-based function space $\mathcal{F}_{i}$, in particular see Remark IV.2. The intricate details of the theory of such Sobolev function spaces will not be needed for our immediate purposes in what follows (compare with Remark V.3.)

Physically, the multiplication of the global excitation field $\mathbf{F}(\mathbf{r})$ by $\psi_{i}(\mathbf{r})$ in constructions like (28) above and (29) below effectively "localizes" (in the topological sense) the field into a smaller compact subdomain, namely the support of the "topological localization basis function" $\psi_{i}(\mathbf{r})$ itself. Moreover, because the $C^{p}$ functions $\psi_{i}(\mathbf{r})$ have compact support $\operatorname{supp}\left\{\psi_{i}\right\} \subset U_{i}$, it follows that $\mathcal{F}\left(U_{i}\right)$ is effectively a local Sobolev space on $U_{i}$ [159].

Remark V.3. We can alternatively define a less complicated function space on $U_{i}$ by

$\mathcal{F}^{\prime}\left(U_{i}\right):=\left\{\psi_{i}(\mathbf{r}) \mathbf{F}(\mathbf{r}), \mathbf{r} \in U_{i} \mid C^{p}\right.$ sup-norm function space $\}$,

where the sup-norm is given by

$$
\left\|\psi_{i}(\mathbf{r}) \mathbf{F}(\mathbf{r})\right\|:=\sup _{\mathbf{r} \in \operatorname{supp}\left\{\psi_{i}\right\}}\left[\psi_{i}(\mathbf{r}) \mathbf{F}(\mathbf{r})\right] .
$$

In the case of $\mathcal{F}^{\prime}\left(U_{i}\right)$, one may further consider only $C^{p}$ vector excitation fields $\mathbf{F}(\mathbf{r})$. The choice of which linear function space to work with depends on the particular application under consideration. In what follows, we further simplify notation by writing $\mathcal{F}_{i}$ instead of $\mathcal{F}\left(U_{i}\right)$ whenever the partition of unity differential atlas coordinate patches $U_{i}$ are used.

\section{Direct Construction of Bundle Homomorphism as Gener- alization of Linear Operators in Electromagnetic Theory}

We now demonstrate how the material constitutive relations in conventional (local) electromagnetic theory may be absorbed into a new structure, the bundle homomorphism, which is the most natural generalization of linear operators in local electromagnetism taking us into the enlarged stage of the generic nonlocal medium's superspace formalism. In the future, these bundle homomorphisms may be discretized using topological numerical methods, e.g., see [166]. In what follows, we focus on the rigorous exact construction using the technique of partition of unity, which allows computations going from local to global domains. ${ }^{18}$

1) The Basic Definition of the Nonlocal Material Banach (Fiber) Bundle Superspace: The initial step in formally defining the proposed nonlocal MTM bundle superspace is the following disjoint union construction:

Definition V.1. (Preliminary Definition of the Bundle Superspace) Let the MTM superspace be denoted by $\mathcal{M}$, which is also called the total bundle space. We define this space as the disjoint union of all spaces $\mathcal{F}_{i}$ of the form:

$$
\mathcal{M}:=\left\{\left(\mathbf{r}, \mathcal{F}_{i}\right) \mid \forall i \in I, \mathbf{r} \in U_{i} \subset \mathcal{D}\right\} .
$$

\footnotetext{
${ }^{18}$ The discretization of the nonlocal MTM bundle homomorphism itself is outside the scope of the present work and will be addressed elsewhere.
}

Associated with $\mathcal{M}$ is a surjective map

$$
p: \mathcal{M} \rightarrow \mathcal{D},
$$

which "projects" the fiber onto its corresponding point in the base manifold $\mathcal{D}$, i.e., $p((\mathbf{r}, \mathcal{F})):=\mathbf{r}$.

Remark V.4. In mainstream literature, the fiber bundle is defined slightly differently. Indeed, the fiber of $\mathcal{M}$ at $\mathbf{r} \in \mathcal{D}$ is defined as the set $p^{-1}(\mathbf{r})$ provided the map $p$ is given as part of the bundle data. This is how fiber bundles are often introduced in the mathematical literature. However, in this paper, we construct the bundle data starting with the physicsbased topological structure (21). The map $p$ is called the projection of the vector bundle $\mathcal{M}$ onto its base space $\mathcal{D}$. Moreover, from now on we will also use the notation $\mathcal{F}_{\mathbf{r}}$ to denote the fiber $p^{-1}(\mathbf{r})$. By construction, it should be clear that $p^{-1}(\mathbf{r})=\mathcal{F}_{i}$ iff $\mathbf{r} \in U_{i}$. From the topological viewpoint, the MTM superspace $\mathcal{M}$ locally appears like a product space $U_{i} \times \mathcal{F}_{i}$. In other words, the map $p$ should behave locally as a conventional projection operator; i.e., in a local domain $U_{i}$, the material's total bundle space $\mathcal{M}$ is isomorphic to $U_{i} \times \mathcal{F}_{i}$, and $p\left(U_{i} \times \mathcal{F}_{i}\right)$ should be isomorphic to $U_{i}$.

In order to complete the specification of the nonlocal MTM superspace, we next construct the linear function space $X_{i}$ defined by

$$
\begin{aligned}
X_{i}:=\left\{\psi_{i}\left[\phi_{i}^{-1}(\bar{x})\right] \mathbf{F}\left[\phi_{i}^{-1}(\bar{x})\right],\right. \\
\left.\bar{x} \in B_{a} \mid \text { Sobolev function space }\right\},
\end{aligned}
$$

which is the Sobolev space of $W^{p, 2}\left(B_{a}\right)$ functions on the Euclidean 3-ball $B_{a}$. Here, each function is defined with respect to the local coordinates $\bar{x}:=\phi^{-1}(\mathbf{r})$, where $\mathbf{r} \in U_{i}$. In fact, it should be straightforward to deduce from the above that there exists maps

$$
\tau_{i}: p^{-1}\left(U_{i}\right) \rightarrow U_{i} \times X_{i},
$$

for all $i \in I$, that are isomorphisms (diffeomorphism in our case), where such diffeomorphism may be expressed by

$$
\forall i \in I: p^{-1}\left(U_{i}\right) \cong U_{i} \times \mathcal{F}_{i} .
$$

The fact that (34) is such an isomorphism follows from the definitions of the spaces $\mathcal{F}_{i}$ and $X_{i}$ by (28) and (33), respectively, and from the fact that each $\phi_{i}$ is a diffeomorphism from $U_{i}$ into $\mathbb{R}^{3}$ (or equivalently the unit 3-ball $B_{a}$ with radius a.) We further note that by construction the diffeomorphism $\tau_{i}$ satisfies

$$
\operatorname{proj}_{1} \circ \tau_{i}=p,
$$

where $\operatorname{proj}_{1}$ is the standard projection map defined by $\operatorname{proj}_{1}(x, y):=x$.

Finally, if we restrict $\tau_{i}$ to $p^{-1}(\mathbf{r})$, the resulting map

$$
\left.\tau_{i}\right|_{p^{-1}(\mathbf{r})}: p^{-1}(\mathbf{r}) \rightarrow\{\mathbf{r}\} \times X_{i}
$$

is a (linear) topological vector space isomorphism from $\mathcal{F}_{\mathbf{r}}$ to $X_{i}$, i.e.,

$$
\forall i \in I, \mathbf{r} \in U_{i}: \mathcal{F}_{\mathbf{r}} \cong X_{i} .
$$

Remark V.5. The charts $\left(U_{i}, \tau_{i}\right)$ are called trivialization covering of the vector bundle $\mathcal{M}$. They provide a coordinate 
representation of local patches of the vector bundle. (The global topology of the bundle, however, is rarely trivial [155].) Since here all maps are $C^{p}$ smooth, $\tau_{i}$ are also called smooth trivialization maps. The complete derivations of the diffeomorphism (35) and the topological vector space isomorphism (38) are straightforward but lengthy and the full proofs are omitted.

Consider now two patches $U_{i}$ and $U_{j}$ with $U_{i} \cap U_{j} \neq \varnothing$. By restricting $\tau_{i}$ and $\tau_{j}$ to $U_{i} \cap U_{j}$, two diffeomorphisms

$$
\begin{aligned}
\tau_{i}: p^{-1}\left(U_{i} \cap U_{j}\right) & \rightarrow\left(U_{i} \cap U_{j}\right) \times X_{i}, \\
\tau_{j}: p^{-1}\left(U_{i} \cap U_{j}\right) & \rightarrow\left(U_{i} \cap U_{j}\right) \times X_{j},
\end{aligned}
$$

are obtained, which in turns implies that

$$
\left(U_{i} \cap U_{j}\right) \times X_{i} \cong\left(U_{i} \cap U_{j}\right) \times X_{j},
$$

or $X_{i} \cong X_{j}$ as expected. In particular, it can be shown that the composition map

$$
\tau_{j} \circ \tau_{i}^{-1}:\left(U_{i} \cap U_{j}\right) \times X_{i} \rightarrow\left(U_{i} \cap U_{j}\right) \times X_{j}
$$

possesses the simple form

$$
\tau_{j} \circ \tau_{i}^{-1}(\mathbf{r}, \mathbf{F})=(\mathbf{r}, g(\mathbf{r}) \mathbf{F}) .
$$

Here, $\mathbf{F} \in X_{i}$ and $g \in \mathrm{L}\left(X_{i}, X_{j}\right)$, where $\mathrm{L}\left(X_{i}, X_{j}\right)$ is the space of linear operators from $X_{i}$ to $X_{j}$ [19]. In particular, $g(\mathbf{r})$ is a $C^{p}$-Banach space isomorphism.

Remark V.6. In the mathematical literature, the smooth maps $\tau_{j} \circ \tau_{i}^{-1}$ are called the vector bundle transition maps. They are essential technical tools for computing global data by starting from local data then gluing them together.

We have now succeeded in directly constructing a specialized smooth Banach vector bundle $(\mathcal{M}, \mathcal{D}, \tau, p)$ consisting of the nonlocal MTM's total fiber bundle space $\mathcal{M}$, base 3manifold $\mathcal{D}$ of the MTM, a set of smooth trivialization charts $\tau_{i}, i \in I$, and a projection map $p$. The base manifolds $\mathcal{D}$ itself is described by a differential atlas $\left(U_{i}, \phi_{i}\right)$ associated to the partition of unity $\left(U_{i}, \psi_{i}\right), i \in I$ as per our discussion in Sec. V-B above.

2) The Nonlocal Material Fiber Bundle Homomrphism: At this point, we need to describe how the evaluation process of the electromagnetic response field (23) may be formulated within the new enlarged framework of the fibered superspace $\mathcal{M}$. The most obvious method is to introduce a new vector bundle with the base space being the same base space $\mathcal{D}$, but with the fibers now taken as the complex Hilbert space $\mathbb{C}^{3}$. This is a well-known vector bundle, which we denote by $\mathcal{R}$, and call the range vector bundle. Formally, the structure of this vector bundle is written as $\left(\mathcal{R}, \mathcal{D}, \tau^{\prime}, p^{\prime}\right)$, where $\tau^{\prime}$ and $p^{\prime}$ are its own smooth trivialization and projection maps. The source vector bundle is taken as $\mathcal{M}$.

The physical process of exciting a nonlocal electromagnetic medium can now be understood as follows:

1) The material domain is mathematically modeled by the Banach bundle $\mathcal{M}$. The response of the medium is to be sought at some point $\mathbf{r} \in \mathcal{D}$.

2) The bundle structure $\mathcal{M}$ will associate a linear function space at $\mathbf{r}$, namely the fiber $p^{-1}(\mathbf{r})$, which is a Banach space of functions defined on the region $U_{i}$ is the model of the excitation field $\mathbf{F}(\mathbf{r})$ after being restricted (topologically localized) to the EM nonlocal domain $U_{i}{ }^{19}$

3) A vector bundle homomorphism (to be formally defined shortly) will map one element of this fiber function space, namely, the particular excitation field $\mathbf{F}(\mathbf{r}), \mathbf{r} \in$ $U_{i}$, to its value in the fiber isomorphic to $\mathbb{C}^{3}$ at $\mathbf{r}$ in the range vector bundle $\mathcal{R}$.

We will need now a precise definition for maps between bundle superspaces. Formally, the is given by [161], [162]:

Definition V.2. (Bundle Homomorphism) A (smooth) bundle homomorphism over a common base space $\mathcal{D}$ shared between the two vector bundles $\mathcal{M}$ and $\mathcal{R}$ is defined by the (smooth) map:

$$
\mathcal{L}: \mathcal{M} \rightarrow \mathcal{R}
$$

satisfying $p^{\prime} \circ \mathcal{L}=p$. Moreover, the restriction of $\mathcal{L}$ to each fiber $p^{-1}(\mathbf{r})$ induces a linear operator on the vector space of that fiber [161]. In effect, the following diagram

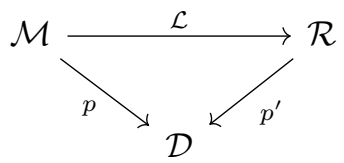

is commutative.

Remark V.7. Because $\mathcal{M}$ and $\mathcal{R}$ share the same base manifold $\mathcal{D}$, the action of the map $\mathcal{L}$ is effectively reduced to how it acts on each fiber $p^{-1}(\mathbf{r})$ as a linear operator.

Now, since the Banach space $X_{i}$ is isomorphic to $p^{-1}(\mathbf{r})$, we will express $\mathcal{L}$ by giving its expression locally in each element $U_{i} \subset \mathcal{D}$ of the open cover $\left\{U_{i}, i \in I\right\}$. In particular, we define the local action using the source and range bundles' trivialization maps $\tau_{i}$ and $\tau_{i}^{\prime}$ by the intuitively obvious formula:

$$
\tau_{i}^{\prime} \circ \mathcal{L}_{\omega} \circ \tau_{i}^{-1}: U_{i} \times X_{i} \rightarrow U_{i} \times \mathbb{C}^{3},
$$

with

$$
\tau_{i}^{\prime} \circ \mathcal{L}_{\omega} \circ \tau_{i}^{-1}:=\left(\mathbf{r}, \mathcal{L}_{i, \omega} \mathbf{F}\right), \mathbf{F} \in X_{i},
$$

where

$$
\mathcal{L}_{i, \omega}: X_{i} \rightarrow \mathbb{C}^{3}
$$

is the linear operator defined by

$$
\mathcal{L}_{i, \omega}(*)=\int_{U_{i}} \mathrm{~d}^{3} r^{\prime} \overline{\mathbf{K}}\left(\mathbf{r}, \mathbf{r}^{\prime} ; \omega\right) \cdot(*),
$$

in which '*' stands for an element of the smooth Banach function space $X_{i}$.

Therefore, within the frequency-domain formulation of this paper, the operator $\mathcal{L}$ will leave every point in the base space $\mathcal{D}$ unchanged while mapping each smooth function on $U_{i}$ (component of the total electromagnetic excitation field, see below) into its complex vector value in $\mathbb{C}^{3}$ at $\mathbf{r} \in U_{i}$.

\footnotetext{
${ }^{19}$ Note that in local electromagnetism, $U_{i}$ is essentially a point $\mathbf{r}$ and hence the excitation field $\mathbf{F}$ is defined on the conventional space $\mathcal{D}$ instead of the Banach bundle space $\mathcal{M}$.
} 
Physically, $\mathcal{L}_{i}$ models a (topologically) localized "piece" of the global electromagnetic material operator mapping excitation fields $\mathbf{F}((\mathbf{r})$ to response fields $\mathbf{R}(\mathbf{r})$, where the entire physics here is restricted to the EM nonlocal subdomain $U_{i}$. The global operator itself is assembled by gluing together all these small pieces using the partition of unity technique as we endeavour to show next.

3) Computing Global Data Starting from Local Data: The final step is tying up together the fundamental source Banach bundle $\mathcal{M}$, range bundle $\mathcal{R}$ and the EM nonlocal microdomain physics space (21). The essential ingredients of the physics of nonlocal EM field-matter interaction are encoded in the geometrical construction of the collection of microdomains $\mathcal{V}(\mathcal{D})=\left\{V_{\mathbf{r}}, \mathbf{r} \in D\right\}$, and the excitation fields $\mathbf{F}(\mathbf{r})$ defined on them, i.e., the sets $\mathcal{V}(\mathcal{D})$ and the (excitation) function spaces $\mathcal{G}(\mathcal{D})$ combined together in one space, the Banach bundle $\mathcal{M}$.

So far, the vector bundle homomorphism $\mathcal{L}$ introduced above takes care of excitation fields supported on the open sets $U_{i}, i \in I$. However, these are mathematical fundamental building blocks used to construct the source vector bundle $\mathcal{M}$. The question now is to how to extend the description of nonlocal EM response operators for excitation fields applied to the entire physical cluster of EM nonlocality microdomains $\left\{V_{\mathbf{r}}, \mathbf{r} \in \mathcal{D}\right)$. As mentioned before, it is the partition of unity $\left(U_{i}, \psi_{i}\right), i \in I$, what will make this expansion of the topological formulation possible.

To see this, let us consider an electromagnetic field $\mathbf{F}(\mathbf{r})$ interacting with a nonlocal medium extended over the manifold $\mathcal{D}$. Our goal is to compute the response field $\mathbf{R}(\mathbf{r})$, that is, at point $\mathbf{r}$. The fundamental idea of EM nonlocality is that to know the response at one point $\mathbf{r}$, one must know the excitation field in a entire open set $V_{\mathbf{r}}$, a topological neighborhood of $\mathbf{r}$, and that in general this microdomain will change depending on the position $\mathbf{r}$. The goal now is to find $\mathbf{R}(\mathbf{r})$ using the vector bundle map $\mathcal{L}$ defined by (43) starting from the data:

1) Region $V_{\mathbf{r}}$,

2) Vector field $\mathbf{F}(\mathbf{r})$ acting on $V_{\mathbf{r}}$.

To accomplish this, we exploit the properties of the partition of unity functions $\psi_{i}$ (Lemma V.1) for expanding the excitation field $\mathbf{F}(\mathbf{r})$ over all patches $U_{i}$ covering $V_{\mathbf{r}}$, resulting in

$$
\mathbf{F}\left(\mathbf{r}^{\prime} ; \omega\right)=\sum_{i \in I_{\mathbf{r}}} \psi_{i}\left(\mathbf{r}^{\prime}\right) \mathbf{F}_{i}\left(\mathbf{r}^{\prime} ; \omega\right)
$$

where (27) was used. The truncated function $\mathbf{F}_{i}$ is equal to $\mathbf{F}(\mathbf{r})$ only if $\mathbf{r} \in U_{i}$ and zero elsewhere, i.e., we have

$$
\mathbf{F}_{i}\left(\mathbf{r}^{\prime} ; \omega\right):=\left\{\begin{array}{cl}
\mathbf{F}\left(\mathbf{r}^{\prime} ; \omega\right), & \mathbf{r} \in U_{i} \\
0, & \mathbf{r} \notin U_{i}
\end{array}\right.
$$

Recall that according to Lemma V.1, the set $I_{\mathbf{r}}$ is defined as the collection of indices $i \in I$ of all $U_{i}$ having the point $\mathbf{r}$ in their common set intersection and is always finite.

The main construction should now become clear. While each truncated sub-field $\mathbf{F}_{i}$ fails to be differentiable (it is not even continuous), the multiplication by $\psi_{i}(\mathbf{r})$ fixes this problem. In fact, each function $\psi_{i}\left(\mathbf{r}^{\prime}\right) \mathbf{F}_{i}\left(\mathbf{r}^{\prime} ; \omega\right)$ is a smooth component of the total excitation field $\mathbf{F}$ with support fully contained inside the coordinate patch $U_{i}$, i.e., $\operatorname{supp}\left\{\mathbf{F}_{i}\right\} \subset U_{i}$.
Consequently, the vector bundle map constructed in (43) can be applied to each such component field. From (44)-(46) and (48), the following can be deduced:

$$
\mathbf{R}(\mathbf{r} ; \omega)=\sum_{i \in I_{\mathbf{r}}} \mathcal{L}_{i, \omega}\left[\psi_{i}\left(\mathbf{r}^{\prime}\right) \mathbf{F}_{i}\left(\mathbf{r}^{\prime} ; \omega\right)\right]
$$

Finally, using (47), we arrive at our main superspace map theorem:

Theorem V.2. (Global Superspace Bundle Map) For the fiber bundle superspace $\mathcal{M}$ of the nonlocal MTM on the manifold $\mathcal{D}$ with material response tensor $\overline{\mathbf{K}}$, the response and excitation fields $\mathbf{R}$ and $\mathbf{F}$ can be related to other other via the global bundle map:

$$
\mathbf{R}(\mathbf{r} ; \omega)=\sum_{i \in I_{\mathbf{r}}} \int_{U_{i}} \mathrm{~d}^{3} r^{\prime} \overline{\mathbf{K}}\left(\mathbf{r}, \mathbf{r}^{\prime} ; \omega\right) \cdot \psi_{i}\left(\mathbf{r}^{\prime}\right) \mathbf{F}_{i}\left(\mathbf{r}^{\prime} ; \omega\right),
$$

where $\psi_{i}, i \in I$, are the partition of unity basis functions subordinated to the $\mathcal{D}$-atlas $\left(U_{i}, \phi_{i}\right), i \in I$.

Physically, Theorem V.2 states that the source bundle $\mathcal{M}$, $\mathcal{R}$, and the response map $\mathcal{L}$ provide a skeleton through which the total response to any EM excitation field defined on an arbitrary EM nonlocality microdomain can be computed. By aggregating all such microdomains constituting the microstructure of nonlocality of the MTM under consideration, the electromagnetism of the medium can be computed and reformulated in the Banach fiber-bundle superspace $\mathcal{M}$ instead of the position space $\mathcal{D}$. In this way, the vector bundle formalism for electromagnetic nonlocality is essentially complete and the connection between the purely mathematical fiber superspace and the physical microdomain structures is secured by (51).

\section{Interlude: The Fiber Bundle Algorithm: SUMMARY AND TRANSITION TO APPLICATIONS}

We now summarize the fiber bundle superspace construction by providing the algorithm derived in the previous Section. Our main goal here is to highlight that the entire construction is based on estimating the EM nonlocality microdomain set $\mathcal{V}(\mathcal{D})=\left\{V_{\mathbf{r}}, \mathbf{r} \in \mathcal{D}\right\}$ associated with the nonlocal MTM domain $\mathcal{D}$. This data can be obtained only through physical theory and/or measurement. However, once available, the construction of the fibered space proceeds in a computationally well-determined manner. We first summarize the algorithm then provide few remarks preparing for some computational examples in latter sections.

In Fig. 4 we show two distinct points $\mathbf{r}_{1}, \mathbf{r}_{2} \in \mathcal{D}$ and their associated microdomains $V_{\mathbf{r}_{1}}$ and $V_{\mathbf{r}_{2}}$, respectively. From the locally finite subcover $\left\{U_{i}\right\}_{i \in I}$ subordinated to $\mathcal{V}(\mathcal{D})=$ $\left\{V_{\mathbf{r}}, \mathbf{r} \in \mathcal{D}\right\}$ we highlight $U_{i} \subseteq V_{\mathbf{r}_{1}}$ and $U_{j} \subseteq V_{\mathbf{r}_{2}}$, where it is possible in general that $V_{\mathbf{r}_{1}} \cap V_{\mathbf{r}_{2}} \neq \emptyset$ and $U_{i} \cap U_{j} \neq \emptyset$ as indicated in the Figure itself. For the partition of unity $\left(U_{i}, \psi_{i}\right)_{i \in I}$ suppordinated to the open cover $\left\{U_{i}\right\}_{i \in I}$, we also depict the two compact sets $S_{i}:=\operatorname{supp}\left\{\psi_{i}(\mathbf{r})\right\}$ and $S_{i}:=\operatorname{supp}\left\{\psi_{i}(\mathbf{r})\right\}$ forming the support of the corresponding partition of unity functions.

The nonlocal MTM superspace algorithm itself is summarized in Algorithm 1. Once the microdomain dataset $\mathcal{V}(\mathcal{D})$ 


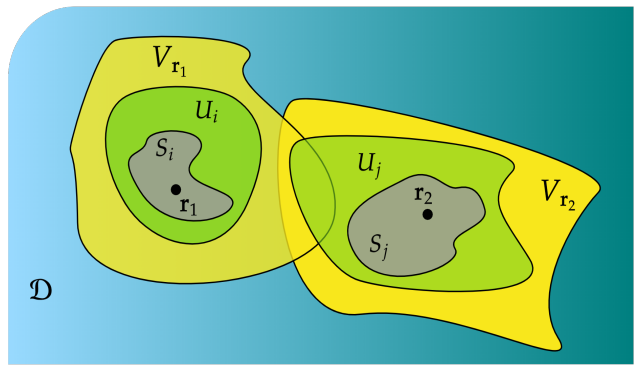

Fig. 4: An example illustrating the various topological microstructures involved in modeling a generic nonlocal material. The microdomains $V_{\mathbf{r}_{1}}, V_{\mathbf{r}_{2}} \in \mathcal{V}(\mathcal{D})$ are open sets and belong to the nonlocal microstructure of the MTM $\mathcal{D}$. The open sets $U_{i}$ and $U_{j}$ are the corresponding coordinate sets and partition of unity functions $\left\{\psi_{i}\right\}_{i \in I}$ 's domains subordinated to $V_{\mathbf{r}_{1}}$ and $V_{\mathbf{r}_{2}}$, respectively. The compact sets $S_{i}$ and $S_{j}$ are defined by $S_{i}:=\operatorname{supp}\left\{\psi_{i}(\mathbf{r})\right\}$ and $S_{i}:=\operatorname{supp}\left\{\psi_{i}(\mathbf{r})\right\}$.

is given, the construction proceeds automatically using the partition of unity basis functions $\left(U_{i}, \psi_{i}\right)_{i \in I}$. The latter may be computed directly in terms of the standard bump functions, see [161], [162], [165] and also Remark V.1.

Because of the fundamental importance of the EM nonlocality microdomain structure $\mathcal{V}(\mathcal{D})$, we will devote Sec. VII entirely to a quantitative practical example illustrating the origin of these microdomains and how they may be estimated in practice. In the subsequent sections, we also explore the usefulness of the homomorphism construction for reformulating boundary-value problems in nonlocal electromagnetic theory and also provide some hints for possible other current and future applications.

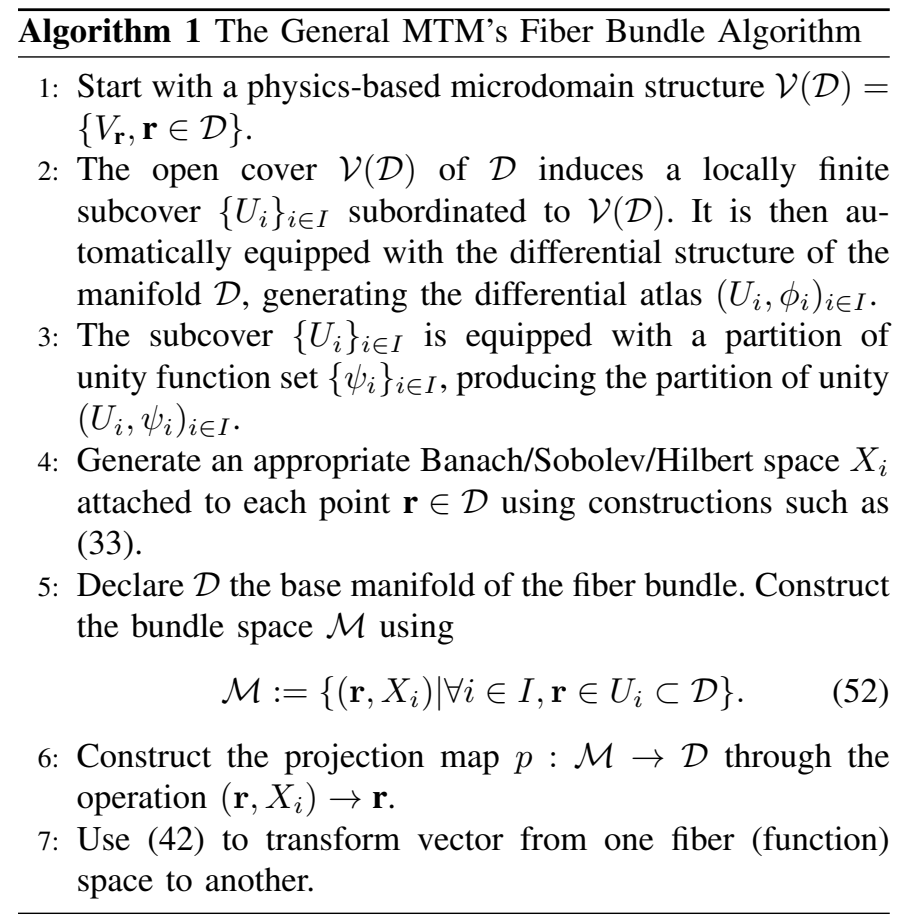

\section{Application to Real-Life Material Systems: ESTIMATING THE MICRODOMAIN TOPOLOGY OF ISOTROPIC SPATIALLY-DISPERSIVE MEDIA}

Here, a concrete example involving spatially-dispersive isotropic media is considered where the intention is to provide an outline of how the intricate fiber-bundle type topological fine structure (the topology of microdomains attached to each point explored above) may be estimated in practice.

\section{A. The Electromagnetic Model of Nonlocal Isotropic Domains}

One of the simplest - yet still demanding and interesting nonlocal media is the special case of isotropic, homogeneous, spatially-dispersive, but optically inactive domains [2]. In this case, very general principles force the generic expression of the material response tensor to acquire the concrete form [3], [4], [126]:

$$
\overline{\mathbf{K}}(\mathbf{k}, \omega)=K^{\mathrm{T}}(k, \omega)(\overline{\mathbf{I}}-\hat{k} \hat{k})+K^{\mathrm{L}}(k, \omega) \hat{k} \hat{k},
$$

where $k:=|\mathbf{k}|, \hat{k}:=\mathbf{k} / k$, and $\mathbf{k}$ is the wavevector (spatialfrequency) of the field. The first term in the RHS of (53) represents the transverse parts of the response function, while the second term is clearly the longitudinal component, with behaviour captured by the generic functions $K^{\mathrm{T}}(k, \omega)$ and $K^{\mathrm{L}}(k, \omega)$, respectively. ${ }^{20}$ The tensorial forms involving the dyads $\hat{k} \hat{k}$, however, are imposed by the formal requirement of the need to satisfy the Onsager symmetry relations in the absence of external magnetic fields [4].

Using a proper microscopic theory, ultimately quantum theory, it is possible in general to derive fundamental expressions for the transverse and longitudinal components of the response functions in (53) [2]-[4], [45], [46], [48]. These forms are often obtained in the following way:

1) First, fundamental theory is deployed to derive analytical expressions for $K^{\mathrm{T}}\left(k, \omega ; \mathbf{r}^{\prime}\right)$ and $K^{\mathrm{L}}\left(k, \omega ; \mathbf{r}^{\prime}\right)$.

2) Afterwards, depending on the concrete values of the various physical parameters that enter into these expressions, e.g., frequency, temperature, molecular charge/mass/spin, density, etc, the obtained analytical expressions are expanded in power series with the proper number of terms.

3) The expression of the dielectric tensor function is then put in the form of either a polynomial or rotational polynomial in $\mathbf{k}$.

A concrete example will be given in Sec. VII-C to illustrate the use of such physics-based dielectric function for the case of exciton-polaritons in semiconductors.

\section{B. A Topological Coarse-Graining Model for Inhomogeneous Nonlocal Material Domains}

We now describe a method that can help transitioning from the generic form (53) valid for homogeneous nonlocal domains to the inhomogeneous medium situation developed throughout

\footnotetext{
${ }^{20}$ Even for isotropic materials, the response tensor $\overline{\mathbf{K}}$ remains a tensor. This is due to the manner in which the equivalent dielectric function is defined using the Fourier transform instead of the conventional multipole approach, e.g., see [4], [45], [121].
} 
this paper where nonlocality cannot be captured by a simple global dependence of the dielectric function on $\mathbf{k}$. However, instead of working with the full nonlocal function $\overline{\mathbf{K}}\left(\mathbf{r}, \mathbf{r}^{\prime}\right)$, an alternative simplified model is proposed which we name the topological coarse-graining model. The idea is as follows. Consider a global material domain $\mathcal{D}$, which is an open 3manifold, say an open subset of $\mathbb{R}^{3}$ that may be either simply connected or disconnected. ${ }^{21}$ The material is nonlocal and inhomogeneous. At each point $\mathbf{r} \in \mathcal{D}$, a microdomain, i.e., and open set $V_{\mathbf{r}} \subset \mathcal{D}$, is assigned. The medium is locally isotropic and homogeneous in the sense that within each microdomain we can describe the response to an external field excitation $\mathbf{E}$ by means of a relation similar to (24), namely:

$$
\mathbf{D}(\mathbf{r} ; \omega)=\varepsilon_{0} \int_{V_{\mathbf{r}}} \mathrm{d}^{3} r^{\prime} \overline{\mathbf{K}}\left(\mathbf{r}-\mathbf{r}^{\prime} ; \omega\right) \cdot \mathbf{E}\left(\mathbf{r}^{\prime} ; \omega\right) .
$$

That is, the only difference between (54) and (24) is that in the former we use the correct form of homogeneous nonlocality $\overline{\mathbf{K}}\left(\mathbf{r}-\mathbf{r}^{\prime} ; \omega\right)$ instead of $\overline{\mathbf{K}}\left(\mathbf{r}, \mathbf{r}^{\prime} ; \omega\right)$. Moreover, we have put the proper response and excitation fields $\mathbf{D}(\mathbf{r})$ and $\mathbf{E}(\mathbf{r})$ and inserted the free space permitvity $\varepsilon_{0}$.

It may be understood now that as a topological coarsegraining process, the original inhomogeneous nonlocal medium ultimately described by the material tensor $\overline{\mathbf{K}}\left(\mathbf{r}, \mathbf{r}^{\prime} ; \omega\right)$ is subdivided into "small topological cells," the microdomains $V_{\mathbf{r}}, \mathbf{r} \in \mathcal{D}$, such that each "topological cell" in itself behaves as a homogeneous nonlocal isotropic subdomain and can hence be described by the form (54) with the material tensor itself taking the (topologically) locally correct form (53). This can be considered a quasi-local model where the global domain is EM nonlocal but in small region (cells) it behaves like an EM local medium, e.g., see [12].

Remark VII.1. The term local is used in this paper in two senses. The first sense is the physical one in which local is set against physical nonlocality, which includes spatial dispersion (EM local/nonlocal.) On the other hand, in topology, a local property is that which holds in a small open neighbourhood of a given point, in our case the topological microdomain $V_{\mathbf{r}}$. The distinction between the two technical senses of the same term should always be clear from the context. In the few cases when there is a risk of confusion, we say topologically local to emphasize the second meaning above from EM local. (see also Remark III.4.)

Our key objective here is to use the simple estimation of the size of nonlocal domains (spheres) outlined above in order to build the topological content of the microdomain structure developed in the discussion started in Sec. IV. For example, consider a point $\mathbf{r}_{1}$, which provides a label for one of the micro-cells we may deploy for creating a coarse-grained model for the inhomogeneous medium. To be more specific, let us construct the topological open ball defined by

$$
V_{\mathbf{r}}=B\left(\mathbf{r}, a_{\mathbf{r}}\right):=\left\{\mathbf{r} \in \mathbb{R}^{3} \mid d\left(\mathbf{r}, \mathbf{r}^{\prime}\right)<a_{\mathbf{r}}\right\},
$$

where $a_{\mathbf{r}} \in \mathbb{R}^{+}$is a number quantifying the smallness of this "nonlocality ball" centered at $\mathbf{r}^{\prime}$ and $d$ is the distance metric.

\footnotetext{
${ }^{21}$ For instance, by introducing holes into a simply-connected domain in order to make the latter disconnected.
}

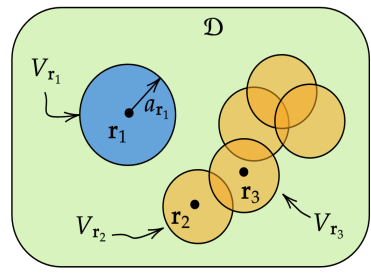

Topological Coarse-Graining Model

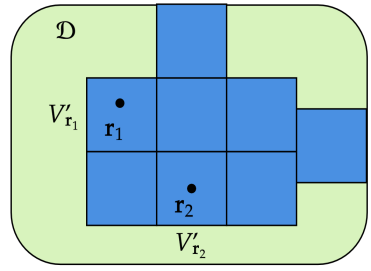

Conventional Coarse-Graining Model
Fig. 5: Topological coarse-graining model for an inhomogeneous nonlocal material domain $\mathcal{D}$ (left) in comparison with a conventional coarse-graining process (right). The topological microdomains constitute an open cover of the domain in the sense that $\mathcal{D}=\bigcup_{\mathbf{r} \in \mathcal{D}} V_{\mathbf{r}}$, which is the obvious generalization of (22). Note how the topological approach allows overlapping microdomains, e.g., between microdomains $V_{\mathbf{r}_{2}}$ and $V_{\mathbf{r}_{3}}$. The technique of the partition of unity will take care of electromagnetic data "repeated" in such regions of overlap by assigning proper weights that always sum to unity at each point in $\mathbf{r} \in \mathcal{D}$.

The number $a_{\mathbf{r}}$ will be determined later based on the actual physics of the problem. Next, the fine-grained topological microdomain structure can be constructed by aggregating all these balls in order to produce a coarse-graining of the overall inhomogeneous nonlocal material domain $\mathcal{D}$. The choice of the shape of the microdomain $V_{\mathbf{r}}$ as a sphere $B\left(\mathbf{r}, a_{\mathbf{r}}\right)$ defined by (55) is justified by our earlier assumption that the material is (topologically) locally isotropic. However, note that globally the electromagnetic processes needs not behave as in isotropic domains.

Fig. 5 provides an illustration of the two processes employing i) the proposed topological coarse-graining model utilizing the set of balls $V_{\mathbf{r}}, \mathbf{r} \in \mathcal{D}$ (left), and ii) the conventional paradigm where the unit cells are nonoverlapping (right). As can be seen from the diagram, in the topological approach, there exists an open set (microdomain) $V_{\mathbf{r}}$ attached to each point $\mathbf{r} \in \mathcal{D}$ such that nearby microdomains may overlap with each other, i.e., the set

$$
\cup_{\mathbf{r}_{1}, \mathbf{r}_{2} \in \mathcal{D}}\left[V_{\mathbf{r}_{1}} \cap V_{\mathbf{r}_{2}}\right]
$$

is not necessarily empty. On the other hand, the conventional approach to coarse-graining (right) involves subdomains like $V_{\mathbf{r}_{1}}^{\prime}$ and $V_{\mathbf{r}_{2}}^{\prime}$ that are nonoverlapping, leading to a grid-like structures or "tiling up" of the material domain $\mathcal{D}$ where in general no holes are left. In both approaches each type of subdomains, whether $V_{\mathbf{r}}$ or $V_{\mathbf{r}}^{\prime}$, is assumed to be homogeneous. The disadvantage of the conventional approach is that abrupt change in electromagnetic properties when transitioning between every two neighboring subdomains often requires imposing a boundary condition between them for the purpose of arriving at a correct computational assessment of the physics inside the material domain $\mathcal{D}$. On the other hand, this problem does not exist in the topological approach (left) because the microdomains are allowed to overlap and common regions between overlapping microdomains are treated correctly using the partition of unity basis functions as described in Sec. V-C. 


\section{Concrete Example: Resonant Nonlocal Semiconductor Do- mains (Exciton-Polariton Interactions)}

We provide here a concrete application of the topological coarse-graining algorithm proposed above. The specific nonlocal metamaterial is a semiconductor with dielectric function exhibiting a single strong resonant exciton transition at frequency $\omega=\omega_{\mathrm{e}}$.

Remark VII.2. Excitons were introduced by Frenkel very early in the history of condensed-matter physics [167], [168] and were further developed by other researchers such as Wannier [169]. In the late 1950s, excitons were brought into the picture of light-matter interaction through the concept of exciton-polariton [139], which will be defined below. Pekar [139], Ginzburg [137], and others [58], [170]-[172] affirmed the nonlocal approach to exciton-polariton materials by explicitly highlighting the strong impact of spatial dispersion near excitonic resonances. The subject of excitons is vast and multidisciplinary. For extensive treatments covering various applications in physics, chemistry, and technology, see [4], [44], [173]-[176].

1) Origin of EM Nonlocality in Excitonic Semiconductors: In order to understand the particular nonlocal model to be presented in Sec. VII-C2, let us first briefly explain the relevant physics of exciton-polariton interactions and why they can lead to strong nonlocal response. In direct-bandgap semiconductor the minimum of the conduction band is aligned along the maximum of the valance band, allowing electronic transitions from lower (unexcited) to excited bands upon interaction with external EM fields. ${ }^{22}$ No free charged carriers are assumed, in contrast to metals and plasma. An electron exiting the valance band after the absorption of an external photon will leave behind a hole, which acts as an independent quasiparticle that can travel throughout the material in the form of a collective excitation [167], [168], [177], [178]. The exciton is defined as a coupled pair composed of the two bound states of the electron and hole. Here, both electrons and holes must be understood as "dressed" particles (quasiparticles) with effective mass and charge different from those of the bare (noninteracting) particle [179]. We may apply the Bohr model to the exciton (electron-hole pair) with simple modifications: First, the electron mass must be replaced by the reduced exciton mass $m_{\mathrm{r}}:=m_{\mathrm{el}} m_{\mathrm{h}} /\left(m_{\mathrm{el}}+m_{\mathrm{h}}\right)$, where $m_{\mathrm{el}}$ and $m_{\mathrm{h}}$ are the electron and hole masses, respectively. ${ }^{23}$ Second, due to the screening of Coulomb attraction by the dielectric medium, the effective electron charge $e^{-}=-e$ should be replaced by $e^{-} / \sqrt{\varepsilon_{0}}$, where $\varepsilon_{0}$ is the static dielectric constant. From this it follows that the exciton binding energy $E_{\mathrm{b}}$ is given by $E_{b}=m_{\mathrm{r}} e^{4} /\left(2 \hbar^{2} \varepsilon_{0}^{2}\right)$. Therefore, the total energy needed to

\footnotetext{
${ }^{22}$ For insulating semiconductors of the II-VI and III-V groups, exciton transitions occur in the visible or near-ultraviolet range of the electromagnetic spectrum. By engineering the material/metamaterial's parameters, these transition frequencies can be shifted.

${ }^{23}$ The numerical values of $m_{\mathrm{el}}$ and $m_{\mathrm{h}}$ are determined by the curvature of the conduction and valance bands, respectively, and hence they follow from accurate quantum mechanical calculations of the band structure, see for example [44], [180], [181].
}

create an exciton state is given by

$$
\hbar \omega_{\mathrm{e}}=E_{\mathrm{g}}-E_{\mathrm{b}}
$$

where $E_{\mathrm{g}}$ is the semiconductor bandgap energy. ${ }^{24}$

They key to the origin of nonlocality is the scenario when the excitation photon has an energy $\hbar \omega$ that is greater than the minimum exciton energy (57). In the case where $\hbar \omega>$ $\hbar \omega_{\mathrm{e}}$, the excess energy will be transformed into kinetic energy. Due to the conservation of momentum, the wavevector of the exciton is equal to the photon wavevector $\mathbf{k}$ and hence the kinetic energy of the exciton is given by $\hbar \mathbf{k} \cdot \hbar \mathbf{k} / 2 m_{\mathrm{e}}$, where $m_{\mathrm{e}}:=m_{\mathrm{el}}+m_{\mathrm{h}}$ is the translational mass of the exciton in the effective-mass approximation [169]. Consequently, the total exciton energy $E_{\mathrm{e}}$ is given by [182]

$$
E_{\mathrm{e}}(\mathbf{k})=\hbar \omega_{\mathrm{e}}(\mathbf{k})=\hbar \omega_{\mathrm{e}}+\frac{\hbar^{2} k^{2}}{2 m_{\mathrm{e}}} .
$$

The exciton frequency $\omega_{\mathrm{e}}(\mathbf{k})$ then acquires a dependence on $\mathbf{k}$ due to the kinetic energy term. As will be shown below, it is precisely such dependence that eventually leads to the emergence of electromagnetic nonlocality in semiconductors around excitonic resonances when photons couple with excitons.

2) The Nonlocal Exicton-Polariton Model: A Polariton is simply a "photons living inside a dielectric medium." That is, the quantization of any electromagnetic wave inside a dielectric domain is often called polariton instead of photons (sometimes polaritons are called "dressed photons.") An exciton-polariton is a polariton coupled with a mechanical exciton like the electron-hole pair defined above. ${ }^{25}$

It is well known from quantum theory that the dielectric function can be approximated near resonance by the form [4], [59], [137], [138], [170]:

$$
\varepsilon(\mathbf{k}, \omega)=\varepsilon_{0}+\frac{\chi}{k^{2}-\gamma^{2}(\omega)},
$$

where

$$
\chi=4 \pi \frac{\alpha m_{\mathrm{e}}^{\star} \omega_{\mathrm{e}}}{\hbar}, \quad \gamma^{2}(\omega)=\frac{m_{\mathrm{e}}^{\star}}{\hbar \omega_{\mathrm{e}}}\left(\omega^{2}-\omega_{\mathrm{e}}^{2}+\mathrm{i} \omega \Gamma\right) .
$$

Here, $\hbar$ is the reduced Planck constant while $\alpha$ is a kind of oscillator strength and can be different for transverse and longitudinal excitation fields. The effective mass of the exciton is denoted by $m_{\mathrm{e}}^{\star} \cdot{ }^{26}$ On the other hand, the exciton lifetime $\tau_{\mathrm{e}}$ is defined by

$$
\tau_{\mathrm{e}}:=\frac{2 \pi}{\Gamma}
$$

\footnotetext{
${ }^{24}$ In most applications, the binding energy $E_{\mathrm{b}}$ is in the order of meV, while $E_{\mathrm{g}}$ is usually few $\mathrm{eV}$. That is, the energy needed to create an exciton is slightly less than the bandgap energy and typically we have $E_{\mathrm{b}} \ll E_{\mathrm{g}}$. However, it is recommended to include binding energy in some applications for accurate calculations to help explaining the fine structure of measured excitonic transitions.

${ }^{25}$ This is to distinguish them from other types of polaritons such as phononpolaritons defined as polaritons coupled with phonons, the quantum of lattice vibrations [45]

${ }^{26}$ In the effective-mass approximation, a simple way to estimate $m_{\mathrm{e}}^{\star}$ is via the relation $m_{\mathrm{e}}^{\star}=m_{\mathrm{el}}+m_{\mathrm{h}}$, the sum of the effective electron and hole masses introduced in Sec. VII-C1. However, it must be noted that this relation is far from being universal, e.g., it should be modified when there are strong interactions [183], [184].
} 
hence $\Gamma$ can be thought of as the exciton decay or relaxation rate. In this model, possible dependencies of $\Gamma$ and the oscillator strength $\alpha$ on $k$ are ignored. In order to obtain significant nonlocality in the material, the following condition can be imposed:

$$
\Gamma \ll \frac{\hbar k^{2}}{2 m_{\mathrm{e}}^{*}} .
$$

Indeed, it can be shown that under such condition the kinetic energy term in (58) leads to significant nonlocal effects in (59). A way to realize nonlocal semiconducting metamaterials is to use intrinsic semiconductors satisfying (62) by keeping the temperature low and the material pure (undoped) [20].

The model described by (59) and (60) can be viewed as a natural generalization of the local Lorentz model widely utilized to model temporal dispersion in solids and plasma [137], [170]. It represents the simplest nonlocal resonant model with a single strong resonance at a characteristic frequency, here $\omega=\omega_{\mathrm{e}}$. All other off-resonance excitonic transitions are gathered into the background dielectric constant $\varepsilon_{0}$ for simplicity. For frequencies well below $\omega \ll \omega_{\mathrm{e}}$, the exciton-polariton behaves essentially like a photon propagating in a medium with background permitivity $\varepsilon_{0}$. For $\omega \gg \omega_{\mathrm{e}}$, we again recover photons but usually with a background described by $\varepsilon_{\infty}$, the high-frequency limit of permitivity. In general, the difference between the static and high-frequency permitivities is quite small, i.e., $\left|\varepsilon_{0}-\varepsilon_{\infty}\right| \ll \varepsilon_{0}$ and in this paper for simplicity we treat them as equal $\left(\varepsilon_{0} \simeq \varepsilon_{\infty}\right)$ since we are interested in the EM response around a single excitonic resonance and the oscillator strength $\alpha$ in (59) is small. One consequence of this assumption is that the splitting between longitudinal and transverse modes can be neglected. Indeed, since the longitudinal and transverse frequencies $\omega_{\mathrm{L}}$ and $\omega_{\mathrm{T}}$ are related to each other via $\omega_{\mathrm{L}}^{2} / \omega_{\mathrm{T}}^{2}=\varepsilon_{0} / \varepsilon_{\infty}$ [4], then the assumption that $\left|\varepsilon_{0}-\varepsilon_{\infty}\right| \ll \varepsilon_{0}$ is equivalent to neglecting the longitudinal-transverse splitting $\omega_{\mathrm{L}, \mathrm{T}}:=\left|\omega_{\mathrm{L}}-\omega_{\mathrm{T}}\right|$ in the sense of $\omega_{\mathrm{L}, \mathrm{t}} \ll \omega_{\mathrm{T}} \cdot{ }^{27} \mathrm{~A}$ consequence of this is the near equality of the longitudinal and transverse frequencies, which allows us to considerably simplify the mathematical treatment. In addition, assuming that the oscillator strength $\alpha$ in (59) is nearly the same for both the longitudinal and transverse part of the response function, then it follows that we need only work with a single scalar response function, namely the form (59) itself instead of the more general tensorial formula (53). ${ }^{28}$

Nonlocal effects associated with the model (59) emerge from the quantum mechanical nature of exciton-polariton interactions and the need to enforce conservation of energy/momentum as discussed in Sec. VII-C1, leading to the strong dependence on $k$ observed in (59).

Remark VII.3. The model (59) itself may be intuitively derived as follows. A generic oscillator model is the Lorentzian form $1 /\left(\omega_{\mathrm{e}}^{2}-\omega^{2}-\mathrm{i} \Gamma \omega\right)$, which models a large number

\footnotetext{
${ }^{27}$ In typical crystal materials, the ratio $\omega_{\mathrm{L}, \mathrm{T}} / \omega_{\mathrm{T}}$ is already about $10^{-3}$ [4], [44].

${ }^{28}$ It should be noted that there is no loss of generality here. The computational model to be presented shortly allows the estimation of the nonlocal microdomain topology based on a generic model of the form (59). If $\varepsilon^{L}$ and $\varepsilon^{\mathrm{T}}$ are not identical, then the same procedure can be applied to each one of them separately.
}

of physical processes, from lattice vibrations to electronic transitions and many others [3], [45], [126]. Substituting the wavevector-dependent $\omega_{\mathrm{e}}$ expression (58) into the just mentioned Lorentzian form, the expression (59) is immediately obtained after keeping only the quadratic terms of k. ${ }^{29}$ This provides a first explanation of nonlocality as arising from the quantum mechanical requirement of enforcing energy/momentum conservation in photon-exciton interactions.

There is yet another physical explanation of nonlocality. When the exciton mass approaches infinity $\left(m_{\mathrm{e}}^{*} \rightarrow \infty\right)$, the kinetic energy term in (58) drops out and the excitonic dielectric function (59) becomes local. This is why spatial dispersion is sometimes referred to as the "finite-mass model," with some suggestions that the origin of nonlocality in this case is the inertial effects of the exciton [170]. ${ }^{30}$ In what follows, we assume that the effective mass of the exciton is always finite and positive, i.e., $0<m_{\mathrm{e}}^{\star}<\infty$. However, it should be noted that since excitons are collective excitations of solids [167], [168], they may have negative mass [173]. While this will not be pursued here, the negativity of the excitonic mass may be exploited in order to further design and control the EM behaviour of nonlocal MTMs constructed using excitonic semiconductors.

To gain a deeper insight into the various resonance structures of the exciton-polariton response function (59), we rewrite it in the equivalent form

$$
\varepsilon(\mathbf{k}, \omega)=\varepsilon_{0}+\frac{\chi / k_{\mathrm{e}}^{2}}{k^{2} / k_{\mathrm{e}}^{2}+1-\omega^{2} / \omega_{\mathrm{e}}^{2}-\mathrm{i} \omega \Gamma / \omega_{\mathrm{e}}^{2}},
$$

where

$$
k_{\mathrm{e}}:=\frac{2 \pi}{\lambda_{\mathrm{e}}}=\sqrt{\frac{m_{\mathrm{e}}^{\star} \omega_{\mathrm{e}}}{\hbar}}
$$

is called the exciton wavenumber. The wavelength $\lambda_{e}$ is a fundamental resonance spatial scale, which we will refer to as the exciton wavelength and is given by

$$
\lambda_{\mathrm{e}}=\frac{1}{2 \pi} \sqrt{\frac{\hbar}{m_{\mathrm{e}}^{\star} \omega_{\mathrm{e}}}} .
$$

For example, with $\hbar \omega_{\mathrm{e}}=2.5 \mathrm{eV}$ and $m_{\mathrm{e}}^{\star}=0.9 m_{\mathrm{e}}$, where $m_{\mathrm{e}}$ is the electron mass, the exciton wavelength $\lambda_{\mathrm{e}}$ is around $0.0293 \mathrm{~nm}$, which is the same order of magnitude of interatomic spacing. The excitation field wavelength $\lambda$ is at least one order of magnitude larger. Later we will show typical values for the topological microdomain radius $a_{\mathbf{r}}$, see Table II below.

There are several fundamental spatial and temporal scales involved in the process of describing generic nonlocal metamaterial domain $\mathcal{D}$. The excitation field $\mathbf{E}(\mathbf{r})$ itself introduces its own excitation period $T:=2 \pi / \omega$ and spatial scale (wavelength) $\lambda:=2 \pi / k$. On the other hand, the excitonic transition as such is associated with the fundamental transition period $T_{\mathrm{e}}:=2 \pi / \omega_{\mathrm{e}}$ and the spatial scale $\lambda_{\mathrm{e}}:=2 \pi / k_{\mathrm{e}}$. In addition,

\footnotetext{
${ }^{29}$ For a more care full quantum mechanical derivation, see [4], [49], [173].

${ }^{30}$ There is a nice parallelism here with temporal dispersion where the latter is known to arise from the inertial effects of electrons in interaction with radiation fields [45].
} 
the radius of a topological microdomain based at a generic position $\mathbf{r} \in \mathcal{D}$ will be shown latter to be given by a special formula (75). Nonlocality arises from the delicate interplay between all these different spatial and temporal scales. In what follows we will emphasize their relative roles in determining the rich nonlocal microstructure of the material domain while introducing quantitative examples. Table I gives a summary of all these parameters with their meaning explicitly stated.

\begin{tabular}{cccc}
\hline \hline SCALE & TYPE & MEANING & FORMULA \\
\hline$\lambda$ & spatial & excitation field wavelength & $\lambda=2 \pi / k$ \\
$\lambda_{\mathrm{e}}$ & spatial & exciton wavelength & $2 \pi / k_{\mathrm{e}}$ \\
$a_{\mathbf{r}}$ & spatial & microdomain radius & $1 /\left|\gamma^{\prime \prime}\right|$ \\
$T$ & temporal & excitation field period & $2 \pi / \omega$ \\
$\tau_{\mathrm{e}}$ & temporal & exciton lifetime & $2 \pi / \Gamma$ \\
$T_{\mathrm{e}}$ & temporal & exciton period & $2 \pi / \omega_{\mathrm{e}}$ \\
\hline \hline
\end{tabular}

TABLE I: A summary of the various spatial and temporal scales involved in understanding and designing generic nonlocal metamaterials with exciton-polariton resonance-type of nonlocality.

Armed with this list of spatial and temporal scales, we are now better positioned to understand the resonance structure associated with the exciton-polariton nonlocal dielectric function (63). Fig. 6 illustrates two cases of resonance studied with respect to variation in the excitation field wavenumber $k$ (wavelength $\lambda$ ). In order to focus on nonlocality, we only plot the nonlocal response part, which is proportional to $\varepsilon(\mathbf{k}, \omega)-\varepsilon_{0}$. As we can see from the two figures, there is a strong resonance taking place when the ratio $k / k=\lambda_{\mathrm{e}} / \lambda$ becomes comparable in magnitude with the quantities remaining in the denominator. That is, the spatial resonance condition is

$$
k^{2} / k_{\mathrm{e}}^{2}+1-\omega^{2} / \omega_{\mathrm{e}}^{2} \sim \omega \Gamma / \omega_{\mathrm{e}}^{2} .
$$

However, (66) may hold only if the imaginary part of the denominator, i.e., the quantity $\omega \Gamma / \omega_{\mathrm{e}}^{2}$ is very small. Otherwise, since $k$ and $k_{\mathrm{e}}$ are real, the ratio $k / k_{\mathrm{e}}$ can never lead to strong resonance if the relaxation rate $\Gamma$ is sufficiently large. Another way to say that is that strong nonlocal spatial resonance happens when dissipation is small, or when the exciton life time is long enough. This special case of long exciton lifetime is characterized by the condition

$$
\omega \Gamma / \omega_{\mathrm{e}}^{2} \ll\left|1-\omega^{2} / \omega_{\mathrm{e}}^{2}\right| .
$$

In this case, it is evident that the spatial and temporal conditions for nonlocal resonance are related to each other by the simple relation

$$
k^{2} / k_{\mathrm{e}}^{2} \approx \omega^{2} / \omega_{\mathrm{e}}^{2}-1
$$

From this it can be inferred that nonlocal resonances generally occur only for $\omega / \omega_{\mathrm{e}}>1$. In Fig. 6 (left), we can see that for the above-resonance condition of $\omega / \omega_{\mathrm{e}}=1.5$, the nonlocal domain possesses a spatial resonance at roughly $\lambda \approx \lambda_{\mathrm{e}}$. On the other hand, if we operate the material at larger frequency $\omega / \omega_{\mathrm{e}}=2.5$, i.e., well above the exciton transition frequency, then spatial resonance occurs at excitation field wavelengths $\lambda$ considerably smaller than $\lambda_{\mathrm{e}}$.

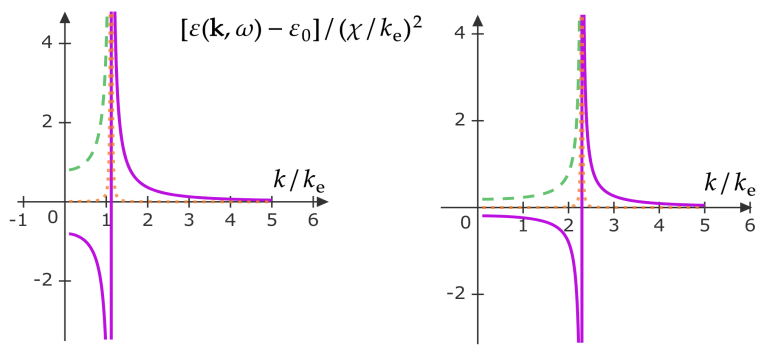

Fig. 6: Nonlocal spatial resonance structure of the excitonpolariton dielectric response as function of the excitation field wavenumber $k$. The normalized response function $(\varepsilon(\mathbf{k}, \omega)-$ $\left.\varepsilon_{0}\right) /\left(\chi / k_{\mathrm{e}}\right)^{2}$ is plotted, where the dashed line is the absolute value, the solid line the real part, while the dotted line is the imaginary part. For both figures $\Gamma / \omega_{\mathrm{e}}=0.01$. Left: $\omega / \omega_{\mathrm{e}}=$ 1.5. Right: $\omega / \omega_{\mathrm{e}}=2.5$.

Finally, we add that when the nonlocal response is plotted as function of $\omega$ instead of $k$, resonance structures similar to Fig. 6 are obtained under the condition (67) since in that case (68) approximately holds. In general, we would expect that for the best operation of the designed nonlocal MTM (maximal nonlocal response), the operating frequency should be made near the exciton transition frequency, i.e., $\omega / \omega_{\mathrm{e}} \approx 1$ since in general $\Gamma$ is never exactly zero and hence the condition (67) seldom hold for all frequencies.

\section{Quantitative Estimation of the EM Nonlocality Microdmain Structure in the Exciton-Polariton Dielectric Nonlocal Model}

The dielectric function in the spatial domain is given by the inverse Fourier transform

$$
\varepsilon\left(\mathbf{r}-\mathbf{r}^{\prime} ; \omega\right)=\mathcal{F}_{\mathbf{k}}^{-1}\{\varepsilon(\mathbf{k}, \omega),
$$

where $\mathcal{F}_{\mathbf{k}}^{-1}$ is the inverse of the forward Fourier transformation defined by (14). We will need the following inverse Fourier transform relation (proved in Appendix B):

$$
\mathcal{F}_{\mathbf{k}}^{-1}\left\{\frac{\chi}{k^{2}-\gamma^{2}(\omega)}\right\}=\frac{\chi}{4 \pi} \frac{e^{\gamma^{\prime \prime}(\omega)\left|\mathbf{r}-\mathbf{r}^{\prime}\right|} e^{-\mathrm{i} \gamma^{\prime}(\omega)\left|\mathbf{r}-\mathbf{r}^{\prime}\right|}}{\left|\mathbf{r}-\mathbf{r}^{\prime}\right|},
$$

where

$$
\begin{aligned}
& \gamma^{\prime}=-\sqrt{\frac{m_{\mathrm{e}}^{\star}}{2 \hbar \omega_{\mathrm{e}}}} \sqrt{\left(\omega^{2}-\omega_{\mathrm{e}}^{2}\right)+\sqrt{\left(\omega^{2}-\omega_{\mathrm{e}}^{2}\right)^{2}+(\omega \Gamma)^{2}}}, \\
& \gamma^{\prime \prime}=-\sqrt{\frac{m_{\mathrm{e}}^{\star}}{2 \hbar \omega_{\mathrm{e}}}} \sqrt{-\left(\omega^{2}-\omega_{\mathrm{e}}^{2}\right)+\sqrt{\left(\omega^{2}-\omega_{\mathrm{e}}^{2}\right)^{2}+(\omega \Gamma)^{2}}} .
\end{aligned}
$$

Therefore by substituting (79) into (69), we arrive at

$$
\varepsilon\left(\mathbf{r}-\mathbf{r}^{\prime} ; \omega\right)=\underbrace{\varepsilon_{0} \delta\left(\mathbf{r}-\mathbf{r}^{\prime}\right)}_{\text {Local response }}+\underbrace{\varepsilon_{\mathrm{NL}}\left(\mathbf{r}-\mathbf{r}^{\prime} ; \omega\right)}_{\text {Nonlocal response }} .
$$

The first terms in the RHS provides the background local response of the medium. On the other hand, all nonlocal effects are relegated to the Green's function

$$
\varepsilon_{\mathrm{NL}}\left(\mathbf{r}-\mathbf{r}^{\prime} ; \omega\right):=\frac{\alpha m_{\mathrm{e}}^{\star} \omega_{\mathrm{e}}}{\hbar} \frac{e^{-\mathrm{i} \gamma^{\prime}(\omega)\left|\mathbf{r}-\mathbf{r}^{\prime}\right|}}{\left|\mathbf{r}-\mathbf{r}^{\prime}\right|} e^{\gamma^{\prime \prime}(\omega)\left|\mathbf{r}-\mathbf{r}^{\prime}\right|}
$$


of the material under investigation. The Green's function (74) is the most fundamental physical quantity needed for the construction of the microdomain structure $\mathcal{D}$ of the nonlocal medium. It has some similarity with the scalar freespace Green's function for radiation fields (spherical waves $\exp \left(\mathrm{i} k\left|\mathbf{r}-\mathbf{r}^{\prime}\right|\right) /\left|\mathbf{r}-\mathbf{r}^{\prime}\right|$, but there are notable differences.

First, we note that (74) exhibits strong dispersive behaviour due to the dependence of $\gamma^{\prime}$ and $\gamma^{\prime \prime}$ on frequency. Second, the existence of the exponential factor $\exp \left(\gamma^{\prime}\left|\mathbf{r}-\mathbf{r}^{\prime}\right|\right)$ makes the Green's function $\varepsilon_{\mathrm{NL}}\left(\mathbf{r}-\mathbf{r}^{\prime} ; \omega\right)$ highly attenuating in spite of the fact that this attenuation is not mainly due to losses. Indeed, as can be seen from (59), dissipation is controlled by the exciton life time $\tau_{\mathrm{e}}$, or equivalently the decay rate $\Gamma$. Dissipation decreases as the life time increases or when $\Gamma$ is small. Fig. 7 illustrates some examples where we plot both $\gamma^{\prime}$ and $\gamma^{\prime \prime}$ as functions of frequency. The frequencydependence behaviour strongly depends on the ratio $\Gamma / \omega_{\mathrm{e}}$, i.e., the ratio between the relaxation frequency and the excitonic transition frequency. For small ratio such as $\Gamma / \omega_{\mathrm{e}}=0.1$, the attenuation constant $\gamma^{\prime \prime}$ is nearly constant for $\omega>\omega_{\mathrm{e}}$, while it assumes higher values for frequencies below the $\omega_{\mathrm{e}}$ as can be seen from Fig. 7(a). This is consistent with high-pass filtering behaviour for this type of resonance where waves are excited for frequencies slightly larger than the cutoff threshold at $\omega_{\mathrm{e}}$. For the propagation constant $\gamma^{\prime}$ at the same relaxation-toexciton transition ratio, Fig. 7(b) shows that it becomes nearly straight line. Such behaviour combined with nearly constant attenuation indicates small dispersion effects. On the other hand, when $\Gamma / \omega_{\mathrm{e}}$ increases, we begin to see strong dispersion effects, manifested by non-constant attenuation and nonlinear phase constant.

In fact, the attenuation captured by the constant $\gamma^{\prime \prime}$ is not merely an expression of dissipation but is the signature of nonlocality in exciton-polariton material domains. The medium response weakens as the distance from the source increases, with the characteristic length scale of this nonlocality radius controlled by $\gamma^{\prime \prime}$. Fig. 8 illustrates the real part of the dielectric function Green's function (74). The ability of the medium to respond to spatially distant sources is graphically illustrated by the spread of the function around the origin $\left|\mathbf{r}-\mathbf{r}^{\prime}\right|=0$. The size of the nonlocal domain is then directly reflected by the rapidity of the decay of the Green's function (74) as one moves away from $\mathbf{r}^{\prime}$, which is the origin here.

\begin{tabular}{|c|c|c|}
\hline$f(\mathrm{THz})$ & $\omega / \omega_{e}$ & $a_{\mathbf{r}}(\mu \mathrm{m})$ \\
\hline 19090 & 0.8 & 0.0003 \\
\hline 21476 & 0.9 & 0.0004 \\
\hline 23862 & 1.0 & 0.0582 \\
\hline 26248 & 1.1 & 7.6670 \\
\hline 35793 & 1.5 & 13.7174 \\
\hline 47724 & 2.0 & 15.9382 \\
\hline 59655 & 2.5 & 16.8674 \\
\hline
\end{tabular}

\begin{tabular}{|c|c|c|}
\hline$\Gamma / \omega_{e}$ & $\omega / \omega_{e}$ & $a_{\mathbf{r}}(\mu \mathrm{m})$ \\
\hline 0.00002 & 1.01 & 2.5834 \\
\hline 0.00020 & 1.01 & 0.2583 \\
\hline 0.00200 & 1.01 & 0.0259 \\
\hline 0.02000 & 1.01 & 0.0028 \\
\hline 0.20000 & 1.01 & 0.0006 \\
\hline 2.0000 & 1.01 & 0.0002 \\
\hline 20.000 & 1.01 & 0.0001 \\
\hline
\end{tabular}

TABLE II: Topological microdomain data at a generic position $\mathbf{r} \in \mathcal{D}$. The exciton transition frequency is $f_{e}=23862 \mathrm{THz}$ $\left(\hbar \omega_{\mathrm{e}}=2.5 \mathrm{eV}\right)$, while $m_{\mathrm{e}}^{\star}=0.9 m_{\mathrm{e}}$. For the left table, $\Gamma / \omega_{\mathrm{e}}=2 \times 10^{-5}$.

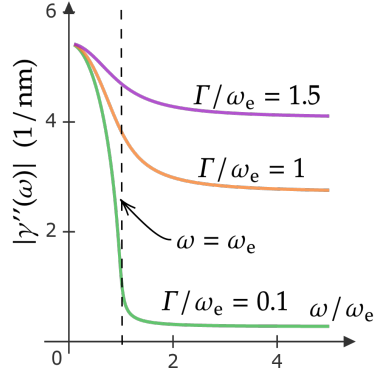

(a)

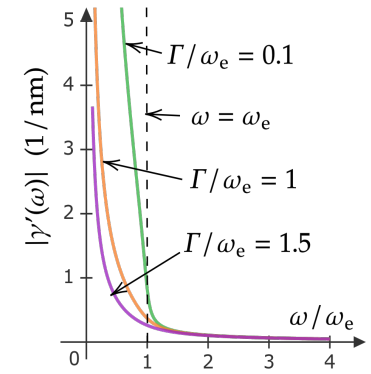

(b)
Fig. 7: Frequency dependence of $\gamma^{\prime}$ and $\gamma^{\prime \prime}$ for several values of the exciton decay rate $\Gamma$. Here, $m_{\mathrm{e}}^{\star}=0.9 m_{\mathrm{e}}$, where $m_{\mathrm{e}}$ is the electron mass. The exciton transition frequency is $\omega_{\mathrm{e}}=$ $3.7977 \times 10^{15} \mathrm{rad} / \mathrm{s}\left(\hbar \omega_{\mathrm{e}}=2.5 \mathrm{eV}\right)$.

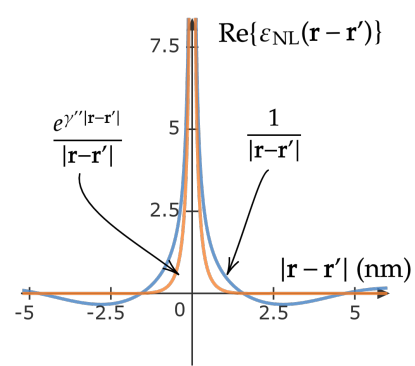

(a)

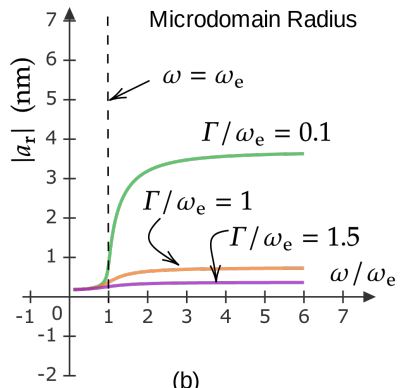

(b)
Fig. 8: (a) Comparison between the real parts of the longrange decay $1 /\left|\mathbf{r}-\mathbf{r}^{\prime}\right|$ of the excitonic nonlocal domain Green's function $\varepsilon_{\mathrm{NL}}\left(\mathbf{r}-\mathbf{r}^{\prime}\right)$ and its full spatial dependence including the exponential decay factor $\exp \left(\gamma^{\prime \prime}\left|\mathbf{r}-\mathbf{r}^{\prime}\right|\right)$ for $\gamma^{\prime}=1 \mathrm{~nm}^{-1}$ and $\gamma^{\prime \prime}=2 \mathrm{~nm}^{-1}$. (b) Frequency dependence of $a_{\mathbf{r}}$, the radious of the topological micordomain $B\left(\mathbf{r}, a_{\mathbf{r}}\right)$ centered at some generic point $\mathbf{r}$ in the nonlocal excotonic material domain $\mathcal{D}$ for several values of the exciton life time $\Gamma^{-1}$. Here, $m_{\mathrm{e}}^{\star}=0.9 m_{e}$, where $m_{\mathrm{e}}$ is the electron mass. The exciton transition frequency is $\omega_{\mathrm{e}}=3.7977 \times 10^{15} \mathrm{rad} / \mathrm{s}$ $\left(\hbar \omega_{\mathrm{e}}=2.5 \mathrm{eV}\right)$.

\section{E. The Locally-Homogeneous Model of Nonlocal Semicon- ducting Domains}

Quasi-inhomogeneous or smoothly-inhomogeneous nonlocal media are some of the simplest possible prototypes of general (inhomogeneous) nonlocal materials where the spatial dispersion model $\varepsilon(\mathbf{k})$ with only a dependence on one variable $\mathbf{k}$ is not adequate to the description of the physics of the problem [12], [39]. In general, there has been quite few investigations aimed at going beyond spatial dispersion in homogeneous media. Examples include inhomogeneous plasma such as those in controlled-fusion reactors [185], cold collisionless magnetoplasma [185], and incommensuratelymodulated superstructures in insulators [12], [186].

Here, we will analyze a simple inhomogeneous model of semiconductors experiencing exciton-polariton transitions 
as outlined above. The EM nonlocal model is locallyhomogeneous in the sense that around each point $\mathbf{r} \mathcal{D}$ there exists a microdomain $V_{\mathbf{r}}$ such the medium can be modeled as homogeneous and spatially dispersive for all $\mathbf{r} \in V_{\mathbf{r}}$ (i.e. the second mention of "locally" here means topological nonlocality.) However, we allow for variations in the spatial dispersion model to take place from one microdomain $V_{\mathbf{r}}$ to another.

We may then estimate the size of each EM nonlocality microdomain by using the exponential law in (74). To see this, let us first generalize to the inhomogeneous setting where at each point $\mathbf{r} \in \mathcal{D}$ the parameters of the excitonPolariton model all become generally functions of position, i.e., we write $\gamma^{\prime}(\mathbf{r}), \gamma^{\prime \prime}(\mathbf{r}), \omega_{\mathrm{e}}(\mathbf{r}), \alpha(\mathbf{r}), m_{\mathrm{e}}^{\star}(\mathbf{r})$, etc, where it is understood that the medium microscopic composition may change from one position to another. The main formula for computing the size (radius) of the topological microdomain balls $V_{\mathbf{r}}=B\left(\mathbf{r}, a_{\mathbf{r}}\right)$ is then given by

$$
a_{\mathbf{r}} \simeq \frac{1}{\left|\gamma^{\prime \prime}(\mathbf{r})\right|},
$$

which is roughly the characteristic localization entailed by an exponential law of the form $\exp \left(-\left|\gamma^{\prime \prime}\right| r^{\prime}\right)$. Using (72), this becomes:

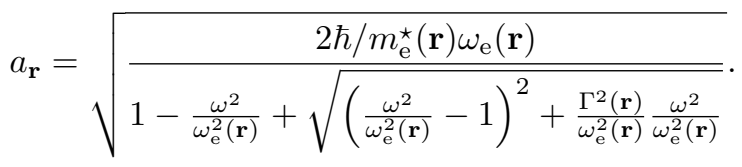

This formula is illustrated in some basic examples in Fig. 8(b) for various values for the ratio $\Gamma / \omega_{\mathrm{e}}$. When this ratio is small, the size of the EM nonlocality domain increases since attenuation becomes smaller. conversely, one may control the size of each EM nonlocality microdomain $V_{\mathbf{r}}$ by modifying the ratio $\Gamma(\mathbf{r}) / \omega_{\mathrm{e}}(\mathbf{r})$ evaluated at that position. This provides a path for an experimental realization of generalized nonlocal MTMs with controlled micro-topological structure. In order to give a view on the numerical values of this structure, Table II provides some relevant microdomain data.

\section{APPlication to FUndamental Theory: ELECTROMAGNETIC BOUNDARY CONDITIONS IN THE FIBER BUNDLE APPROACH}

The well-known tension between nonlocal electromagnetism and material interfaces has been already mentioned several times above. Here, we provide some application of the fiber bundle theory of Sec. V aiming at elucidating the nature of this tension and to suggest some possible new formulation of the problem. The starting point is Fig. 2, where a zoomed-in topological picture based on the general structures explicated in Sec. IV is given. The focus now is on the interface between two generic nonlocal domains $\mathcal{D}_{m}$ and $\mathcal{D}_{m}$. In traditional local electromagnetism, the constitutive relation material tensor $\overline{\mathbf{K}}_{n}$ is exploited to deduce conditions dictating how various electromagnetic field components behave as they cross the $\mathcal{D}_{m} / \mathcal{D}_{m}$ interface. However, even if each $\overline{\mathbf{K}}_{n}\left(\mathbf{r}, \mathbf{r}^{\prime}\right)$ was to be treated as that belonging to a spatially dispersive domain, i.e., by replacing it by $\overline{\mathbf{K}}_{n}\left(\mathbf{r}-\mathbf{r}^{\prime}\right)$, the presence of a boundary completely destroys the translational symmetry of the structure on which the very form of the spatial dispersion nonlocal response tensor $\overline{\mathbf{K}}_{n}\left(\mathbf{r}-\mathbf{r}^{\prime}\right)$ is based. This was very clearly explained in [4], with several proposals for a solution of the electromagnetic problem. For example, since close to the interface it is very obvious that the material tensor must be reverted back to the most general nonlocal form, namely $\overline{\mathbf{K}}_{n}\left(\mathbf{r}, \mathbf{r}^{\prime}\right)$, it was then proposed that one may use the latter form only in a thin region containing the interface on both sides. Outside this region, a gradual transition or a continuous profile (tapering) is introduced to transition from the forms $\overline{\mathbf{K}}_{n}\left(\mathbf{r}, \mathbf{r}^{\prime}\right)$ and $\overline{\mathbf{K}}_{m}\left(\mathbf{r}, \mathbf{r}^{\prime}\right)$ to the spatially dispersive forms $\overline{\mathbf{K}}_{n}\left(\mathbf{r}-\mathbf{r}^{\prime}\right)$ and $\overline{\mathbf{K}}_{m}\left(\mathbf{r}-\mathbf{r}^{\prime}\right)$ characteristic of "bulk" homogeneous material domains [4].

Another proposal is to keep the spatial dispersion profiles $\overline{\mathbf{K}}_{n}\left(\mathbf{r}-\mathbf{r}^{\prime}\right)$ and $\overline{\mathbf{K}}_{m}\left(\mathbf{r}-\mathbf{r}^{\prime}\right)$ everywhere but introduce specialized additional boundary conditions (ABCs) at the interface suitable for the problem at hand. Although this latter approach is neither consistent mathematically nor physically (because of the breakdown of symmetry caused by the presence of an interface), it nevertheless remains popular because - at least in outline - nonlocal electromagnetism is thereby held up in a form as close as possible to familiar local electromagnetic theory methods, especially numerical techniques such as Finite Element Method (FEM) [129] , Method of Moment (MoM) [131], and Finite Difference Time-Domain Method (FDTD) [130], i.e., established full-wave algorithms where it is quite straightforward to replace one boundary condition by another without essentially changing much of the code. ${ }^{31}$

Nevertheless, each of the two approaches discussed above requires a considerable input from microscopic theory, mainly to determine the tapering transition region in the case of the first, and the ABCs themselves in the second. That motivated the third approach, called, the ABC-free formalism, where the relevant microscopic theory was utilized right from the beginning in order to formulate and solve Maxwell's equations. For example, in [46], [47], a global Hamiltonian of the matter-field system is constructed and Maxwell's equations are derived accordingly. In [48], the rim zone (field attached to matter) is investigated using different physical assumptions to understand the transition from nonlocal material domains to vacuum going through the entire complex near-field zone.

We believe that the main common conclusion from all these different formulations is that in nonlocal electromagnetism it is not possible in general to formulate the electromagnetic problem at a fully phenomenological level. In other words, microscopic theory appears to be in demand more often than in the case of systems involving only local materials. However, since all existing solutions use the traditional spatial manifold $\mathcal{D}$ as the main configuration space, the question now is whether the alternative formulation proposed in this paper, the extended fiber bundle approach, may provide some additional insights.

We provide a provisional elucidation of the topological nature of electromagnetism across material interfaces by noting that in Fig. 2, not only the behaviour of the fields $\mathbf{F}(\mathbf{r})$ in the two domains is relevant, but also the entire local topological

\footnotetext{
${ }^{31}$ This is more obvious in FEM or FDTD than MoM.
} 
fiber superspace boundary condition

$$
\mathcal{D}_{m} \rightleftarrows \mathcal{D}_{n} \Rightarrow X_{m} \rightleftarrows X_{n}
$$

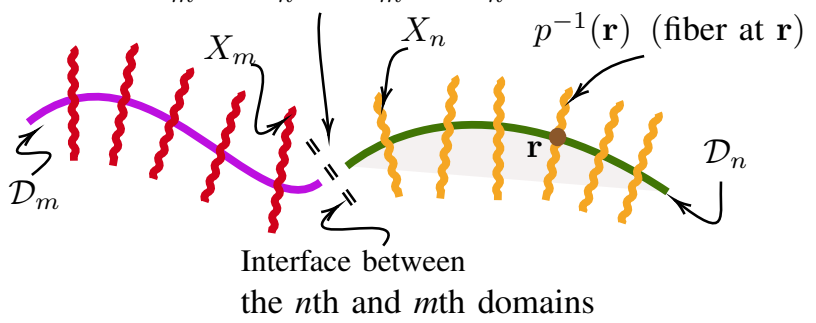

Fig. 9: An abstract representation of the fiber bundle structure behind Fig. 2.

microdomains $V_{\mathbf{r}}$ clustered inside, and in particular how these topological regions with fields on them behave as they move across the boundary. In general topology, boundaries are defined fully in terms of the behaviour of open sets.

We build on this key concept in order to illustrate how the problem of nonlocal electromagnetism across interfaces may be reformulated. First, Fig. 9 provides a finer or more structured picture of the topological structure of Fig. 2 based on replacing the spaces $\mathcal{D}_{m}$ and $\mathcal{D}_{n}$ by the corresponding Banach bundle superspaces $\mathcal{M}_{m}$ and $\mathcal{M}_{n}$, respectively. The thick horizontal curved lines represent the bases spaces $\mathcal{D}_{m}$ and $\mathcal{D}_{n}$, while the wavy vertical lines stands for the fibers spaces $X_{m}$ and $X_{n}$ attached to each point in the corresponding base manifolds. The double discontinuous lines at the "junction" of the two base spaces $\mathcal{D}_{m}$ and $\mathcal{D}_{n}$ indicate the joining together of the two vector bundles $\mathcal{M}_{m}$ and $\mathcal{M}_{n}$.

It should become clear now that since the two nonlocal material domains possess an extra structure, namely that of the fiber superspaces attached to each point in the base space, we must also indicate how the various elements belonging to the Banach function spaces, the fields defined on the microdomains $V_{r}$ in Fig. 2, behave as they cross the boundary separating $\mathcal{D}_{m}$ and $\mathcal{D}_{n}$. One obvious way to do this is to introduce a bundle homomorphism [161] between the two vector bundles $\mathcal{M}_{m}$ and $\mathcal{M}_{n}$ over the interface submanifold $\partial \mathcal{D}_{m n}$ separating $\mathcal{D}_{m}$ and $\mathcal{D}_{n}{ }^{32}$

The motivation behind introducing this bundle homomorphism is to serve as a "boundary condition operator" acting on the fiber bundle nonlocal domains $\mathcal{M}_{m}$ and $\mathcal{M}_{n}$ instead of $\mathcal{D}_{m}$ and $\mathcal{D}_{n}$. We will not go here into constructing this operator in details, but provide instead some additional remarks to illustrate the main idea. The traditional boundary condition applied in the base space will be denoted by $\mathcal{D}_{m} \rightleftarrows \mathcal{D}_{n}$ and is usually spelled out in the form

$$
\begin{aligned}
\lim _{\mathbf{r} \rightarrow \partial \mathcal{D}_{m n}}\left\{\mathbf{F}_{m}(\mathbf{r})-\mathbf{F}_{n}(\mathbf{r})\right\} & =\Gamma_{b_{1}}\left[\mathbf{F}_{m}(\mathbf{r}), \mathbf{F}_{n}(\mathbf{r})\right], \\
\lim _{\mathbf{r} \rightarrow \partial \mathcal{D}_{m n}}\left\{\mathbf{R}_{m}(\mathbf{r})-\mathbf{R}_{n}(\mathbf{r})\right\} & =\Gamma_{b_{2}}\left[\mathbf{R}_{m}(\mathbf{r}), \mathbf{R}_{n}(\mathbf{r})\right],
\end{aligned}
$$

where $\partial \mathcal{D}_{m n}$ is the boundary between $\mathcal{D}_{m}$ and $\mathcal{D}_{n}$. Here, $\Gamma_{b_{1}}$ and $\Gamma_{b_{2}}$ are "base space boundary functions." On the other

\footnotetext{
${ }^{32}$ This mathematical object is similar to the nonlocal response map $\mathcal{L}$ introduced by (43).
}

hand, the fiber bundle elements, i.e., functions defined on on the microdomains $V_{\mathbf{r}}$, are mapped by

$$
X_{m} \rightleftarrows X_{n}: \quad \lim _{\mathbf{r} \rightarrow \partial \mathcal{D}_{m n}}\left(X_{m}-X_{n}\right)=\Gamma_{f}\left[X_{m}, X_{n}\right],
$$

where $\Gamma_{f}$ is a different fiber superspace boundary function. The full formulation is more complex because the boundary condition operator must also be proved to be compatible with the fiber bundle structures and so the entire global topology of $\mathcal{M}_{m}$ and $\mathcal{M}_{n}$ will interact with the effective final electromagnetic boundary condition resulting from this process.

The main relevant conclusion here is that the existence of extra or additional structures in the fiber bundle space of electromagnetic nonlocality makes the need for additional boundary conditions or information coming from the microscopic topological structure very natural. The fiber bundle formalism of nonlocal metamaterials does capture the physics of nonlocal domains joined together through interfaces. The full formulation of the proposed fiber bundle boundary condition homomorphism is beyond the scope of this paper but it is hopped that this initial insight can at least clarify the subject and stimulate further research in the fundamental theory of nonlocal metamaterials.

\section{Further Applications And Future Work}

We give a general outline of several additional possible applications, where some of them highlights the theory developed in this paper. Issues pertinent to fundamental considerations (physical and mathematical) and engineering functions are taken up in Secs. IX-A and IX-B, respectively. ${ }^{33}$

\section{A. Applications to Fundamental Theory and Methods}

1) Limitations on Nonlocal Metamaterials: Maps like $\mathcal{L}$ (43) can be reformulated in the space of vector bundle sections [155], [161], [164], a subject that is extremely well developed in classic differential topology. In fact, the electromagnetic response field function $\mathbf{R}$ itself can sometimes be obtained by working directly with the source bundle superspace $\mathcal{M}$. For example, under some conditions, this can be achieved by replacing each fiber $X_{i}$ by $X_{i} \times \mathbb{C}^{3}$. In this way, the entire electromagnetic nonlocal response problems becomes identical to the investigation of how vector bundle sections interact with the topology of the underlying base manifold $\mathcal{D}$. There is an extremely large literature in differential topology and geometry focused on this problem, especially how local information can be propagated to extend into global structures [19], [156], [161].

The author believes that by starting from local data in a given nonlocal metamaterial domain, e.g., the global shape of the device, the distribution of topological holes, etc, one may then use existing techniques borrowed from differential topology, e.g., the theory of characteristic classes, to determine the allowable EM response functions permissible in principle at the global level. Engineers are typically interested in

\footnotetext{
${ }^{33} \mathrm{All}$ of the coming discussions is kept very brief and only the essential main ideas are given. Some of these applications are expected to be investigated more extensively in the near future.
} 
knowing in advance what the best (or worst) performance measures obtainable from specific topologies are, and hence reformulating the electromagnetism of nonlocal metamaterials in terms of vector bundles could be of help in this respect since it opens a pathway toward a synergy between general topology, physics, and engineering in the field of metamaterials.

2) Numerical Methods: Traditional full-wave numerical methods are sometimes deployed to deal with nonlocal EM materials, often using the additional boundary conditions framework, in spite of the latter's lack of complete generality. At the heart of the traditional approach to numerical methods in local electromagnetism is the concept of operators between linear spaces. However, by reformulating the source space of field-matter interaction in terms of a Banach bundle, it should be possible to reformulate Maxwell's equations to act on this extended geometric superspace instead of the conventional spacetime framework. Instead of the concept of linear operator, we now have the much more general and richer concept of bundle homomorphism developed in details above. Some of the advantages anticipated from such reformulation is the ability to resolve the issue of generalized boundary condition (Sec. VIII). Moreover, since every point belonging to a fiber superspace is in itself a smooth function defined on an entire material sub-microdomain, by building a new system of discretized recursive equations approximating the behaviour of electromagnetic solutions living in the enlarged superspaces $\mathcal{M}$ and $\mathcal{R}$, one may expect deeper understanding of the physics of nonlocality since the topology of the nonlocal interaction regime is explicitly encoded into the geometry of the new expanded solution superspace $\mathcal{M}$ itself. It is also possible that such numerical methods may emerge as more computationally efficient and broader in applicability than the conventional methods rooted in local electromagnetism. One reason for this is that the Banach vector bundle formulation introduced in this paper is quite natural and appears to reflect the underlying physics of nonlocal metamaterials in a direct manner. In recent years, the subject of computational topology has gained momentum and some researchers are now building new numerical methods by exploiting the topological structure of the problems under considerations, e.g., see [166], [187].

\section{B. Other Applications of Nonlocal Metamaterials}

1) Topological photonics: One of the main applications of the proposed vector bundle formalism is that it opens the door for a new way to investigate the topological structure of materials. It has already been noticed that nonlocal EM response is essential in topological photonics, e.g., see [100], [111]. Indeed, since in topological photonics the wavefunction of bosons, usually the Bloch state, is examined over the entirety of momentum space (usually the Brillouin zone), then it is the dependence of the EM response on $\mathbf{k}$ what is at stake, which naturally brings in nonlocal issues. But now since by using our theory we can associate with every nonlocal material a concrete fiber bundle superspace reflecting the rich information about the multiscale topological microdomain structure and the global shape of the material plus the impact of the boundaries separating various material domains, it is natural to examine whether a topological classification of the corresponding fiber bundles may lead to a new way to characterize the topology of materials other than the Chern invariants used extensively in literature. The advantage of the superspace approach in this case is that the complicated topological and geometrical aspects of the boundaries and inhomogeneity in nonlocal media can be encoded very efficiently in the local structure of the material fiber bundle. Using standard techniques in differential topology [155], it should be possible to propagate this local information to the global domain (the entirety of the system), for example by computing fiber the bundle topological invariants like its homology groups [164]. Our approach is then a "duall" to the standard approach since we work on an enlarged configuration space (spacetime or space-frequency), while the mainstream approach operates in the momentum space of the wavefunction.

2) Digital Communications: Nonlocal metamaterials offer a very wide range of potential applications in wireless communications and optical fibers. The basic idea is to introduce specially engineered nonlocal domains either as part of the communication channel (e.g., optical fibers, plasmonic circuits, microwave transmission lines) [150], or as a control structure integrated with existing antennas [121]. Spatial dispersion was also used as a method to engineer wave propagation characteristics in material domains, e.g., see [188] for applications to high-efficiency modulation of free-space EM waves. A general linear partial equation explicating how spatial and temporal dispersion can be jointly exploited to produce zero distortion (e.g., constant negative group velocity) was derived and solved in [22]. The main idea originated from the fact that distortion in communication systems emerge from nonconstant group velocity $\mathbf{v}_{g}:=\nabla_{\mathbf{k}} \omega$. Since $\mathbf{v}_{g}$ is a strong function of the dependence of the material response tensor $\overline{\mathbf{K}}(\mathbf{k}, \omega)$ on both $\mathbf{k}$ and $\omega$, dispersion management equations can be derived for several applications. For example, it was proved in [22] that in simple isotropic spatially dispersive media with high-symmetry, one may obtain exact solutions where the group velocity is constant at an entire frequency band. This happens because while strong temporal dispersion is present (which alone causes strong distortion), the presence of optimized spatially dispersive profiles lead to compensation of distortion, resulting essentially distortionfree channels. There are enormous potentials of research in this exciting area since most practical realizations of nonlocal metamaterials involve complex material response tensors and the relevant mathematics of dispersion engineering is still relatively underdeveloped.

3) Electromagnetic Metamaterials: As early as the 1960s, it was proposed that EM nonlocality can be exploited to produce materials with very unusual properties. For example, in [4], negative refraction materials were noted as one possible application of spatial dispersion where the path toward attaining this goes through controlling the direction of the group velocity vector. Since in nonlocal media power does not flow along the Poynting vector [2], new (higher-order) effects were shown to be capable of generating arbitrary group velocity profiles by carefully controlling the spatial and temporal dispersion profiles. Overall, the ability of spatial dispersion 
to induce higher-order corrections to power flow is a unique added advantage enjoyed by nonlocal metamaterials exhibiting spatial dispersion in addition to normal dispersion. This extra spatial degrees of freedom provided by space was researched, reviewed and highlighted many publications, including for example [52], [73], [80], [102], [114], [121], [132], [145], [189], [190].

4) Near-Field Engineering, Nonlocal Antennas, and Energy Applications: Another interesting application of nonlocality in electromagnetic media is near-field engineering, a subject that has not yet received the attention it deserves. It was observed in [189] that a source radiating in homogeneous, unbounded isotropic spatial dispersive medium may exhibit several unusual and interesting phenomena due to the emergence of extra poles in the radiation Green's function of such domains. Both longitudinal and transverse waves are possible (dispersion relations), and the dispersion engineering equations relevant to finding suitable modes capable of engineering desired radiation field patterns are relatively easy to set and solve. For example, by carefully controlling the modes of the radiated waves, it is possible to shape the near field profile, including total confinement of the field around the antenna even when losses is very small, opening the door for applications like energy harvesting, storage, and retrieval in such media. This subject, however, has been explored only for simple materials so far and mainly at the theoretical level [121]. On the other hand, the subject of far-field radiation by sources embedded into nonlocal media was investigated previously by some authors within the context of plasma domains [34]. Recently, it has been systematized into a general theory for nonlocal antennas with media possessing an arbitrary spatial dispersion profile [191]-[193]. However, no general theory exists for nonlocal media which are inhomogeneous. The superspace formalism proposed in this paper may help stimulate research into this direction.

\section{COnClusion}

We provided a general theoretical and conceptual investigation of nonlocal metamaterials aiming at achieving several goals. First, the subject was reviewed from a new perspective with the intention of introducing it to a wide audience, including engineers, applied physicists, and mathematicians. The various essential ideas behind EM nonlocality were viewed in new light using an abstract field-response model in three dimensions. Next, the fine-grained topological microstructure of nonlocal metamaterials was explicated in details. We introduced EM nonlocality microdomains and showed that they present an important structural topological feature of the physics of nonlocal media. After that, it was proved using differential topology that a natural fiber bundle structure serving as a source space can be constructed. The source fiber bundle was shown to have all the required properties of standard fiber bundles while faithfully reflecting the physics of EM nonlocality microdomains. Eventually, and using the technique of partition of unity, it was proved that the source fiber bundle can be used to construct and compute the material response function over arbitrary microdomains. This was accomplished by building a bundle homomorphism to replace the material tensor linear operators in conventional electromagnetism. The new homomorphism is a generalization of linear operators and can in turn be discretized in the future using suitable methods available in computational topology.

The new fiber bundle superspace formulation suggests that EM nonlocality can be formulated in an alternative way compared with other existing methods that borrow heavily from the electromagnetism of local media. Most importantly, EM nonlocality forces us to consider an entire infinite-dimensional Banach space attached to each point in the conventional 3dimensional space on which the material is defined. This extra or additional structure provides a natural explanation of why traditional boundary conditions often fail to account for the physics of nonlocal metamaterials. Moreover, the fiber bundle theory opens the door for several new applications, including the ability to understand the deep connection between topology and electromagnetism in engineered novel artificial media. Overall, the author proposes that future research in metamaterials will gradually require more extensive collaboration between engineers and mathematicians to explore the full consequences of this organic topology/electromagnetism relation.

\section{APPENDIX}

\section{A. Guide to the Mathematical Background}

We provide an informal overview regarding how to read the mathematical portions of this paper and where to find detailed references that might be needed in order to expand some of the technical proof sketches provided in the main text. We emphasize that in this paper only the elementary definitions of 1) differential manifolds, 2) Banach and Sobolev spaces, 3) vector bundles, and 4) partition of unity are needed to understand the mathematical development.

Differential manifolds. A differential manifold is a collection of fundamental "topological atoms" each composed of an open set $U_{i}$ and a chart $\phi_{i}(x)$, which serves as a coordinate system, basically an invertible smooth map to the Euclidean space $\mathbb{R}^{n}$. That is, locally, every manifold looks like a Euclidean space with dimension $n$. The collection of open sets $U_{i}, i \in I$, where $I$ is an index set, covers this $n$-dimensional manifold. Since some of these open sets are allowed to overlap, the key idea of the differential manifold is that on the overlap region $U_{i} \cap U_{j}$, there exist a smooth reversible coordinate transformation function connecting the coordinates of the same point when expressed in the two (different) languages of the topological atoms $U_{i}$ and $U_{j}$. The key concept of topology is how to propagate information from the local to the global. In this sense, differential manifolds present elementary strucutre allowing us to model this process using the efficient technology of the differential calculus. Only the basic definition of smooth manifolds is required in this paper, which can be found in virtually any book on differential or Reimannian geometry, e.g., see [8], [16], [19], [155], [158], [161], [164], [194].

In Sec. IV, we introduced Sobolev space over the open domain $D$ instead of simply Banach space. However, that was 
done mainly to simplify the technical development and in anticipation of future work. Indeed, in this paper, the fiber bundle $\mathcal{M}$ is referred to just as Banach bundle, not Sobolev bundle for the reason that all our essential results and insights apply to the more general concept of Banach space, which contain Soblev spaces as special case. However, Sobolev spaces are easier to implement and we only invoked here the key definition of the space itself. In particular, none of the other technical properties of Sobolev spaces are needed in the paper. However, since in the future the material bundle space $\mathcal{M}$ is expected to be used to construct solution of Maxwell's equations in new form, Sobolev spaces are projected to play the most important role since they have proved very efficient in analysis. For the basic definition of Sobolev spaces and their applications to partial differential equations in mathematical physics and finite-element method in engineering, we recommend [158]. The subject of Banach manifolds is less commonly treated in literature than finite-dimensional manifolds, but good concise treatments of the topic include [19], [164], [194].

Vector bundles. Fiber bundles, of which vector bundles are special case, are now standard topics in both mathematics (topology, geometry, differential equations), theoretical physics (quantum field theory, cosmology, quantum gravity), and applied physics (condensed-matter physics, many-body problems). For the major importance of vector and fiber bundles within the overall structure of modern fundamental physics, see [8], [16], [18]. In quantum field theory, gauge field theories use vector bundles as essential ingredients in the standard model of particle physics [16]. The increasing importance of methods based on quantum field theory in applications to condensed-matter physics has contributed into making knowledge of fiber bundle techniques useful and more widespread in physical and engineering research, e.g., see the area of Berry phase and the associated gauge connection [24], [111]. The key idea of a vector bundle is to attach an entire vector space to every point on a base manifold. To be more specific, consider a differential manifold $\mathcal{D}$. Each such vector space will be called the fiber at that point. The tangent space of the manifold is the most obvious example of such vector bundles. However, more complicated structures than finite-dimensional tangent spaces can also be encoded by the vector bundle concept. In this paper, we have shown that EM nonlocality can be modeled naturally by considering the Banach space of all fields on the microdomains based at a point in the material configuration space. Fiber bundles then can be seen as highly efficient and economic ways to encapsulate large amount of topological and geometrical data and they lend themselves easily to complex calculations. Very readable technical descriptions of vector bundles can be found in [16], [156], [161], [164].

Partition of unity techniques. These are somehow technical tools used by topologists to propagate information from the local to the global and are quite handy and easy to apply. The main theorems allow moving from one topological atom to another by "gluing" them together using smooth standard domain-division functions. The technique was stated and used only toward the end of Sec. V to write the expansion (48) and can be skipped in first reading of the paper. Partition of unity is usually taught in all topology and some geometry textbooks, e.g., see [19], [156], [161], [164].

\section{B. Computation of the Inverse Fourier Transform (70)}

We start from the standard Fourier transform pair

$\mathcal{F}_{\mathbf{k}}^{-1}\left\{\frac{\chi}{k^{2}-\gamma^{2}(\omega)}\right\}=\frac{\chi}{4 \pi} \frac{e^{-\mathrm{i} \gamma(\omega)\left|\mathbf{r}-\mathbf{r}^{\prime}\right|}}{\left|\mathbf{r}-\mathbf{r}^{\prime}\right|}, \quad \operatorname{Im}\{\gamma(\omega)\}<0$,

where the spatial Fourier transform is defined by (14). The condition $\operatorname{Im}\{\gamma(\omega)\}<0$ is due to the physical requirement that fields don't grow exponentially in passive domains [126]. We also have written $\left|\mathbf{r}-\mathbf{r}^{\prime}\right|$ instead of $|\mathbf{r}|$ in anticipation of the fact that the inverse Fourier transform will produce a Green's function.

Our main task now is to make a proper choice of the correct sign of when taking the square root operation $\sqrt{\gamma^{2}}$. Let us write $\gamma^{2}=\operatorname{Re}\left\{\gamma^{2}\right\}+\mathrm{i} \operatorname{Im}\left\{\gamma^{2}\right\}$. From (60) we have

$$
\operatorname{Re}\left\{\gamma^{2}\right\}=\frac{m_{\mathrm{e}}^{\star}}{\hbar \omega_{\mathrm{e}}}\left(\omega^{2}-\omega_{\mathrm{e}}^{2}\right), \quad \operatorname{Im}\left\{\gamma^{2}\right\}=\frac{m_{\mathrm{e}}^{\star}}{\hbar \omega_{\mathrm{e}}} \omega \Gamma .
$$

On the other hand, $\gamma$ also can take the form

$$
\gamma(\omega)=\gamma^{\prime}(\omega)+\mathrm{i} \gamma^{\prime \prime}(\omega)
$$

where both $\gamma^{\prime}$ and $\gamma^{\prime \prime}$ are real. The goal now is to derive expressions for $\gamma^{\prime}$ and $\gamma^{\prime \prime}$ in terms of $\operatorname{Re}\left\{\gamma^{2}\right\}$ and $\operatorname{Im}\left\{\gamma^{2}\right\}$ with the correct sign since the square root is a many-one function.

To accomplish this, we use the following elementary theorem: Let $x, y, a, b \in \mathbb{R}$. Then the square root of $x+\mathrm{i} y$ is given by

$$
\sqrt{x+\mathrm{i} y}= \pm(a+\mathrm{i} b)
$$

where the following expressions hold

$$
a=\sqrt{\frac{x+\sqrt{x^{2}+y^{2}}}{2}}, \quad b=\frac{y}{|y|} \sqrt{\frac{-x+\sqrt{x^{2}+y^{2}}}{2}} .
$$

Substituting (80) into (83), the following is obtained

$$
\begin{aligned}
a & =\sqrt{\frac{m_{\mathrm{e}}^{\star}}{2 \hbar \omega_{\mathrm{e}}}} \sqrt{\left(\omega^{2}-\omega_{\mathrm{e}}^{2}\right)+\sqrt{\left(\omega^{2}-\omega_{\mathrm{e}}^{2}\right)^{2}+(\omega \Gamma)^{2}}}, \\
b & =\sqrt{\frac{m_{\mathrm{e}}^{\star}}{2 \hbar \omega_{\mathrm{e}}}} \sqrt{-\left(\omega^{2}-\omega_{\mathrm{e}}^{2}\right)+\sqrt{\left(\omega^{2}-\omega_{\mathrm{e}}^{2}\right)^{2}+(\omega \Gamma)^{2}}}
\end{aligned}
$$

Here, we used the calculation $y /|y|=\operatorname{Im}\left\{\gamma^{2}\right\} /\left|\operatorname{Im}\left\{\gamma^{2}\right\}\right|=$ $\omega \Gamma /|\omega \Gamma|=1$, which follows from the fact that $\omega, \Gamma>0$.

It remains now to find the correct signs. From (79), the condition $\gamma^{\prime \prime}=\operatorname{Im}\{\gamma(\omega)\}<0$ must be satisfied. Therefore, we choose the negative sign in (82). The final expressions become $\gamma=-a, \gamma^{\prime \prime}=-b$, and after inserting $\gamma^{\prime}$ and $\gamma^{\prime \prime}$ into (79), the required relation (70) is obtained. 


\section{REFERENCES}

[1] J. Schwinger et al., Classical electrodynamics. Reading, Mass: Perseus Books, 1998

[2] V. L. Ginzburg, The propagation of electromagnetic waves in plasmas. Oxford,New York: Pergamon Press, 1970.

[3] — Theoretical physics and astrophysics. Oxford New York: Pergamon Press, 1979.

[4] V. Agranovich and V. Ginzburg, Crystal optics with spatial dispersion, and excitons. Berlin, Heidelberg: Springer Berlin HeidelbergImprint Springer, 1984

[5] A. S. Shvarts, "On the definition of superspace," Theoretical and Mathematical Physics, vol. 60, no. 1, pp. 657-660, Jul 1984.

[6] J. Barbour, The end of time: the next revolution in physics. Oxford New York: Oxford University Press, 2000.

[7] E. Anderson, The problem of time: quantum mechanics versus general relativity. Cham, Switzerland: Springer, 2017.

[8] R. Penrose, The road to reality: a complete guide to the laws of the universe. New York: Vintage Books, 2007.

[9] B. McClain, A. Niemi, C. Taylor, and L. C. R. Wijewardhana, "Superspace, negative dimensions, and quantum field theories," Phys. Rev. Lett., vol. 49, pp. 252-255, Jul 1982.

[10] M. Castagnino, "Mathematical structure of quantum superspace as a consequence of time asymmetry," Phys. Rev. D, vol. 57, pp. 750-767, Jan 1998.

[11] A. Janner and T. Janssen, "Symmetry of periodically distorted crystals," Phys. Rev. B, vol. 15, pp. 643-658, Jan 1977.

[12] O. S. Kushnir, "Spatial dispersion in incommensurately modulated insulators," Journal of Physics: Condensed Matter, vol. 16, no. 8, pp. 1245-1267, feb 2004.

[13] T. Tsakalakos et al., Modulated structure materials. Dordrecht Boston: M. Nijhoff, 1984.

[14] L. Elcoro and J. M. Perez-Mato, "Superspace description of quasiperiodic structures and the nonuniqueness of superspace embedding," Phys. Rev. B, vol. 54, pp. 12115-12 124, Nov 1996.

[15] A. F. Andreev, "Mesoscopic superconductivity in superspace," Journal of Experimental and Theoretical Physics Letters, vol. 68, no. 8, pp. 673-678, Oct. 1998.

[16] E. Zeidler, Quantum field theory I: Basics in Mathematics and Physics. Springer, 2009.

[17] — Quantum field theory II: Quantum Electrodynamics. Springer, 2006

[18] — Quantum field theory III: Gauge Theory. Springer, 2011.

[19] S. Lang, Introduction to differentiable manifolds. New York: Interscience, 1962.

[20] P. Halevi et al., Spatial dispersion in solids and plasmas. Amsterdam New York: North-Holland, 1992.

[21] V. M. Agranovich and Y. Gartstein, "Spatial dispersion and negative refraction of light," Physics-Uspekhi, vol. 49, no. 10, p. 1029, 2006.

[22] S. Mikki and A. Kishk, "Electromagnetic wave propagation in nonlocal media: Negative group velocity and beyond," Progress In Electromagnetics Research B, vol. 14, pp. 149-174, 2009.

[23] G. Qi, "Optical beams in media with spatial dispersion," Chinese Physics Letters, vol. 20, no. 1, pp. 64-67, dec 2002.

[24] A. Altland and B. Simmons, Condensed matter field theory. Leiden: Cambridge University Press, 2010.

[25] A. Morro, "Optical activity and spatial dispersion," Phys. Rev. E, vol. 56, pp. 1124-1128, Jul 1997.

[26] V. Agranovich and V. Yudson, "On phenomenological electrodynamics of gyrotropic media," Optics Communications, vol. 9, no. 1, pp. 58-60, Sep 1973.

[27] S. A. Trigger, "Equilibrium radiation in a plasma medium with spatial and frequency dispersion," Physica Scripta, vol. 95, no. 7, p. 075504, jun 2020.

[28] R. Esquivel-Sirvent, C. Villarreal, W. L. Mochán, A. M. ContrerasReyes, and V. B. Svetovoy, "Spatial dispersion in Casimir forces: a brief review," Journal of Physics A: Mathematical and General, vol. 39, no. 21, pp. 6323-6331, May 2006.

[29] M. Fabrizio and A. Morro, Electromagnetism of continuous media: mathematical modelling and applications. Oxford: Oxford University Press, 2003.

[30] A. Eringen, Nonlocal continuum field theories. New York: Springer, 2002.

[31] P. T. Leung and R. Chang, "Reciprocity in nonlocal nano-optics," Journal of Optics A: Pure and Applied Optics, vol. 10, no. 7, p. 075201, May 2008.
[32] H. Y. Xie, P. T. Leung, and D. P. Tsai, "General proof of optical reciprocity for nonlocal electrodynamics," Journal of Physics A: Mathematical and Theoretical, vol. 42, no. 4, p. 045402, dec 2008.

[33] _ _ "Reciprocity theorem for nonlocal optics: completion of proof and application to spectroscopic analysis," Journal of Optics, vol. 12, no. 3, p. 035006, feb 2010.

[34] D. D. Bareev and V. G. Gavrilenko, "On the lorentz lemma for media with spatial dispersion and its application to calculation of the radiation fields of given sources," Radiophysics and Quantum Electronics, vol. 52, no. 10, pp. 735-739, Oct 2009.

[35] M. Tokman, E. Westerhof, and M. Gavrilova, "Wave power balance in resonant dissipative media with spatial and temporal dispersion," Nuclear Fusion, vol. 43, no. 11, pp. 1295-1304, Nov 2003.

[36] M. Amooshahi and F. Kheirandish, "Electromagnetic field quantization in an anisotropic magnetodielectric medium with spatial-temporal dispersion," Journal of Physics A: Mathematical and Theoretical, vol. 41 , no. 27 , p. 275402 , jun 2008.

[37] S. Y. Buhmann, D. T. Butcher, and S. Scheel, "Macroscopic quantum electrodynamics in nonlocal and nonreciprocal media," New Journal of Physics, vol. 14, no. 8, p. 083034, aug 2012.

[38] S. A. R. Horsley and T. G. Philbin, "Canonical quantization of electromagnetism in spatially dispersive media," New Journal of Physics, vol. 16, no. 1, p. 013030, Jan 2014.

[39] A. A. Balakin and E. D. Gospodchikov, "Operator formalism for permittivity tensor in smoothly inhomogeneous media with spatial dispersion," Journal of Physics B: Atomic, Molecular and Optical Physics, vol. 48, no. 21, p. 215701, Sep 2015.

[40] S. Y. Nastyshyn, I. M. Bolesta, S. A. Tsybulia, E. Lychkovskyy, Z. Y. Fedorovych, D. Y. Khaustov, Y. Ryzhov, P. I. Vankevych, and Y. A. Nastishin, "Optical spatial dispersion in terms of Jones calculus," Phys. Rev. A, vol. 100, p. 013806, Jul 2019.

[41] S. Raza, S. I. Bozhevolnyi, M. Wubs, and N. A. Mortensen, "Nonlocal optical response in metallic nanostructures," Journal of Physics: Condensed Matter, vol. 27, no. 18, p. 183204, April 2015.

[42] B. Adolph, V. I. Gavrilenko, K. Tenelsen, F. Bechstedt, and R. Del Sole, "Nonlocality and many-body effects in the optical properties of semiconductors," Phys. Rev. B, vol. 53, pp. 9797-9808, Apr 1996.

[43] M. Dommermuth and N. Schopohl, "On the theory of light propagation in crystalline dielectrics," Journal of Physics Communications, vol. 2, no. 7, p. 075012, Jul 2018.

[44] S. I. Pekar, Crystal optics and additional light waves. Menlo Park, Calif: Benjamin/Cummings Pub. Co, 1983.

[45] Y. A. Ilinskii and L. Keldysh, Electromagnetic response of material media. New York: Springer Science+Business Media, 1994.

[46] K. Cho, Optical response of nanostructures: microscopic nonlocal theory. Berlin New York: Springer, 2003.

[47] — Reconstruction of macroscopic Maxwell equations: a single susceptibility theory. Berlin, Germany: Springer, 2018.

[48] O. Keller, Quantum Theory of Near-Field Electrodynamics. Berlin New York: Springer, 2011.

[49] Y. Toyozawa, Optical processes in solids. Cambridge, UK New York: Cambridge University Press, 2003.

[50] L. Brillouin, Wave propagation in periodic structures, electric filters and crystal lattices. New York: Dover Publications, 1953.

[51] S. Mikki and A. Kishk, "A symmetry-based formalism for the electrodynamics of nanotubes," Progress In Electromagnetics Research, vol. 86, pp. 111-134, 2008.

[52] T. V. Mechelen and Z. Jacob, "Nonlocal topological electromagnetic phases of matter," Phys. Rev. B, vol. 99, p. 205146, May 2019.

[53] M. Born and K. Huang, Dynamical theory of crystal lattices. Oxford New York: Clarendon Press Oxford University Press, 1988.

[54] S. Mikki and A. Kishk, "Derivation of the carbon nanotube susceptibility tensor using lattice dynamics formalism," Progress In Electromagnetics Research B, vol. 9, pp. 1-26, 2008.

[55] M. G. Silveirinha, "Additional boundary conditions for nonconnected wire media," New Journal of Physics, vol. 11, no. 11, p. 113016, Nov 2009.

[56] R. J. Churchill and T. G. Philbin, "Electromagnetic reflection, transmission, and energy density at boundaries of nonlocal media," Phys. Rev. B, vol. 94, p. 235422, Dec 2016.

[57] G. S. Agarwal, D. N. Pattanayak, and E. Wolf, "Electromagnetic fields in spatially dispersive media," Phys. Rev. B, vol. 10, pp. 1447-1475, Aug 1974.

[58] A. A. Maradudin and D. L. Mills, "Effect of spatial dispersion on the properties of a semi-infinite dielectric," Phys. Rev. B, vol. 7, pp. 2787-2810, Mar 1973. 
[59] F. Forstmann, "Optics in spatially dispersive media: The energy theorem and the boundary conditions," Zeitschrift für Physik B Condensed Matter and Quanta, vol. 32, no. 4, pp. 385-393, Dec 1979.

[60] M. Fang, Z.-X. Huang, W. E. I. Sha, X. Y. Z. Xiong, and X.-L. Wu, "Full hydrodynamic model of nonlinear electromagnetic response in metallic metamaterials (invited paper)," Progress In Electromagnetics Research, vol. 157, pp. 63-78, 2016.

[61] V. M. Galynsky, A. N. Furs, and L. M. Barkovsky, "Integral formalism for surface electromagnetic waves in bianisotropic media," Journal of Physics A: Mathematical and General, vol. 37, no. 18, pp. 5083-5096, April 2004.

[62] C. Forestiere, A. Capretti, and G. Miano, "Surface integral method for second harmonic generation in metal nanoparticles including both local-surface and nonlocal-bulk sources," J. Opt. Soc. Am. B, vol. 30 , no. 9, pp. 2355-2364, Sep 2013.

[63] K. Mnasri, A. Khrabustovskyi, M. Plum, and C. Rockstuhl, "Retrieving effective material parameters of metamaterials characterized by nonlocal constitutive relations," Phys. Rev. B, vol. 99, p. 035442, Jan 2019.

[64] P. Vallejos, T. Johnson, and T. Hellsten, "An iterative method to include spatial dispersion for waves in nonuniform plasmas using wavelet decomposition," Journal of Physics: Conference Series, vol. 775, p. 012016, Nov 2016.

[65] P. Vallejos, T. Hellsten, and T. Jonsson, "A numerical tool based on FEM and wavelets to account for spatial dispersion in ICRH simulations," Journal of Physics: Conference Series, vol. 1125, p. 012020, Nov 2018.

[66] C. JiSheng and L. JiaRong, "The boundary value problem and the nonlinear spatial dispersion in collisionless quark-gluon plasma," Communications in Theoretical Physics, vol. 34, no. 2, pp. 251-260, Sep 2000.

[67] T. Musienko, V. Rudakov, and L. Solovev, "On the application of Kramers-Kronig relations to media with spatial dispersion," Journal of Physics: Condensed Matter, vol. 1, no. 37, pp. 6745-6753, Sep 1989

[68] F. Z. Goffi, K. Mnasri, M. Plum, C. Rockstuhl, and A. Khrabustovskyi, "Towards more general constitutive relations for metamaterials: A checklist for consistent formulations," Phys. Rev. B, vol. 101, p. 195411, May 2020.

[69] A. Konovalenko, J. A. Reyes-Avendaño, A. Méndez-Blas, F. Cervera E. Myslivets, S. Radic, J. Sánchez-Dehesa, and F. Pérez-Rodríguez, "Nonlocal electrodynamics of homogenized metal-dielectric photonic crystals," Journal of Optics, vol. 21, no. 8, p. 085102, Jul 2019.

[70] L. Zheng and G. Qi-Huang, "Surface polaritons in a wire medium with spatial dispersion," Chinese Physics Letters, vol. 25, no. 1, pp. 298-301, Jan 2008.

[71] P. A. Belov, R. Marqués, S. I. Maslovski, I. S. Nefedov, M. Silveirinha, C. R. Simovski, and S. A. Tretyakov, "Strong spatial dispersion in wire media in the very large wavelength limit," Phys. Rev. B, vol. 67, p. 113103, Mar 2003.

[72] M. G. Silveirinha, "Nonlocal homogenization model for a periodic array of $\epsilon$-negative rods," Phys. Rev. E, vol. 73, p. 046612, April 2006.

[73] C. R. Simovski, P. A. Belov, A. V. Atrashchenko, and Y. S. Kivshar, "Wire metamaterials: Physics and applications," Advanced Materials, vol. 24, no. 31, pp. 4229-4248, Jul. 2012.

[74] A. Moradi, "Spatial nonlocality in the infrared absorption spectra of polar semiconductor nanospheres," Semiconductor Science and Technology, vol. 30, no. 11, p. 115003, Sep 2015

[75] J. R. Maack, N. A. Mortensen, and M. Wubs, "Size-dependent nonlocal effects in plasmonic semiconductor particles," EPL (Europhysics Letters), vol. 119, no. 1, p. 17003, Jul 2017.

[76] T. Dong and X. Ma, "Electromagnetic scattering by gyrotropic semiconductor spheres when considering spatial dispersion," Journal of Physics D: Applied Physics, vol. 51, no. 28, p. 285302, jun 2018.

[77] Y. A. Eremin, L. Mädler, and T. Wriedt, "Influence of the nonlocal effect on the optical properties of nonspherical plasmonic semiconductor nanoparticles," Computational Mathematics and Modeling, vol. 31, no. 1, pp. 58-74, Jan 2020.

[78] R. F. Souza, M. A. R. C. Alencar, M. R. Meneghetti, J. Dupont, and J. M. Hickmann, "Nonlocal optical nonlinearity of ionic liquids," Journal of Physics: Condensed Matter, vol. 20, no. 15, p. 155102, mar 2008.

[79] A. Poddubny, I. Iorsh, P. Belov, and Y. Kivshar, "Hyperbolic metamaterials," Nature Photonics, vol. 7, no. 12, pp. 948-957, Nov. 2013

[80] A. Orlov, P. M. Voroshilov, P. A. Belov, and Y. S. Kivshar, "Engineered optical nonlocality in nanostructured metamaterials," Phys. Rev. B, vol. 84, p. 045424, Jul 2011.
[81] M. Becchi, C. Oldano, and S. Ponti, "Spatial dispersion and optics of crystals," Journal of Optics A: Pure and Applied Optics, vol. 1, no. 6 , pp. 713-718, oct 1999.

[82] A. B. Shvartsburg, M. B. Agranat, and O. V. Chefonov, "Nanooptics of gradient dielectric films," Quantum Electronics, vol. 39, no. 10, pp. 948-952, oct 2009.

[83] P. Weissglas, "Spatial dispersion and absorption of waves in bounded low-density plasma," Journal of Nuclear Energy. Part C, Plasma Physics, Accelerators, Thermonuclear Research, vol. 6, no. 3, pp. 251265, January 1964

[84] G. Brodin and M. Marklund, "On the possibility of metamaterial properties in spin plasmas," New Journal of Physics, vol. 10, no. 11, p. 115031, Nov 2008

[85] O. Sakai and K. Tachibana, "Plasmas as metamaterials: a review," Plasma Sources Science and Technology, vol. 21, no. 1, p. 013001, Jan 2012.

[86] C.-Y. Wang and G.-H. Wang, "Spatially nonlocal effects on optical absorption properties in coupled quantum wells with an applied electric field," Chinese Physics B, vol. 23, no. 12, p. 127103, Nov 2014.

[87] K. Ikeda and O. Akimoto, "Steady propagation of a coherent light pulse in a dielectric medium - Part II. the effect of spatial dispersion," Journal of Physics A: Mathematical and General, vol. 12, no. 7, pp. 1105-1120, Jul 1979.

[88] B. Alfassi, C. Rotschild, O. Manela, M. Segev, and D. N Christodoulides, "Nonlocal surface-wave solitons," Phys. Rev. Lett., vol. 98, p. 213901, May 2007.

[89] W.-P. Zhong, L. Yi, R.-H. Xie, M. Belić, and G. Chen, "Robust three-dimensional spatial soliton clusters in strongly nonlocal media," Journal of Physics B: Atomic, Molecular and Optical Physics, vol. 41, no. 2, p. 025402, Jan 2008 .

[90] H. Zhang, D. Xu, and L. Li, "An approximate solution for describing a fundamental soliton in nonlocal nonlinear media," Journal of Optics A: Pure and Applied Optics, vol. 11, no. 12, p. 125203, Sep 2009.

[91] S. Pu, C. Hou, K. Zhan, and Y. Du, "Beam splitters in inhomogeneous nonlocal media," Physica Scripta, vol. 86, no. 2, p. 025404, Jul 2012.

[92] A. Alberucci, M. Peccianti, G. Assanto, A. Dyadyusha, and M. Kaczmarek, "Two-color vector solitons in nonlocal media," Phys. Rev. Lett., vol. 97, p. 153903, Oct 2006.

[93] J. I. Vestgården, P. Mikheenko, Y. M. Galperin, and T. H. Johansen, "Nonlocal electrodynamics of normal and superconducting films," New Journal of Physics, vol. 15, no. 9, p. 093001, Sep 2013.

[94] A. A. A. Jr, G. L. Alfimov, and A. S. Malishevskii, "Nonlocal electrodynamics of josephson vortices in superconducting circuits," Superconductor Science and Technology, vol. 22, no. 2, p. 023001, Jan 2009.

[95] F. Binkowski, L. Zschiedrich, M. Hammerschmidt, and S. Burger, "Modal analysis for nanoplasmonics with nonlocal material properties," Phys. Rev. B, vol. 100, p. 155406, Oct 2019.

[96] Y. Luo, A. I. Fernandez-Dominguez, A. Wiener, S. A. Maier, and J. B. Pendry, "Surface plasmons and nonlocality: A simple model," Phys. Rev. Lett., vol. 111, p. 093901, Aug 2013.

[97] M. Moaied, S. Palomba, and K. Ostrikov, "Quantum plasmonics: longitudinal quantum plasmons in copper, gold, and silver," Journal of Optics, vol. 19, no. 10, p. 105402, Sep 2017.

[98] E. V. G. Ramírez, S. A. S. Hernández, J. J. M. Rodríguez, G. Díaz, M. L. A. Carrasco, and J. A. R. Esqueda, "Nonlocality in Ag nanocubes: dependence on the concentration and incident wavelength," Materials Research Express, vol. 6, no. 12, p. 1250c6, Jan 2020.

[99] J. Gratus, P. Kinsler, M. W. McCall, and R. T. Thompson, "On spacetime transformation optics: temporal and spatial dispersion," New Journal of Physics, vol. 18, no. 12, p. 123010, dec 2016.

[100] M. G. Silveirinha, "Chern invariants for continuous media," Phys. Rev. $B$, vol. 92, p. 125153, Sep 2015.

[101] N. Banerjee, S. Dutta, and D. Roychowdhury, "Chern-Simons superconductor," Classical and Quantum Gravity, vol. 31, no. 24, p. 245005 , Nov 2014.

[102] S. Mikki and A. Kishk, "Electromagnetic wave propagation in dispersive negative group velocity media," in 2008 IEEE MTT-S International Microwave Symposium Digest, June 2008, pp. 205-208.

[103] H. Hapuarachchi, S. D. Gunapala, and M. Premaratne, "Plasmonic metaresonances: harnessing nonlocal effects for prospective biomedica applications," Journal of Physics: Condensed Matter, vol. 31, no. 32, p. 325301, May 2019.

[104] M. A. Gorlach and P. A. Belov, "Nonlocality in uniaxially polarizable media," Phys. Rev. B, vol. 92, p. 085107, Aug 2015. 
[105] T. Skettrup, "Effective optical constants in the treatment of spatial dispersion," Journal of Physics D: Applied Physics, vol. 14, no. 7, pp. 1343-1354, Jul 1981.

[106] J. S. Pérez-Huerta, G. P. Ortiz, B. S. Mendoza, and W. L. Mochán, "Macroscopic optical response and photonic bands," New Journal of Physics, vol. 15, no. 4, p. 043037, April 2013.

[107] A. Ciattoni and C. Rizza, "Nonlocal homogenization theory in metamaterials: Effective electromagnetic spatial dispersion and artificial chirality," Phys. Rev. B, vol. 91, p. 184207, May 2015.

[108] K. Mnasri, A. Khrabustovskyi, C. Stohrer, M. Plum, and C. Rockstuhl, "Beyond local effective material properties for metamaterials," Phys. Rev. B, vol. 97, p. 075439, Feb 2018.

[109] M. Z. Hasan and C. L. Kane, "Colloquium: Topological insulators," Rev. Mod. Phys., vol. 82, pp. 3045-3067, Nov 2010.

[110] S. Raghu and F. D. M. Haldane, "Analogs of quantum-Hall-effect edge states in photonic crystals," Phys. Rev. A, vol. 78, p. 033834, Sep 2008.

[111] T. Ozawa, H. M. Price, A. Amo, N. Goldman, M. Hafezi, L. Lu, M. C. Rechtsman, D. Schuster, J. Simon, O. Zilberberg, and I. Carusotto, "Topological photonics," Rev. Mod. Phys., vol. 91, p. 015006, Mar 2019

[112] L. Lu, J. D. Joannopoulos, and M. Soljačić, "Topological photonics," Nature Photonics, vol. 8, no. 11, pp. 821-829, Oct. 2014.

[113] W.-J. Chen, B. Hou, Z.-Q. Zhang, J. B. Pendry, and C. T. Chan, "Metamaterials with index ellipsoids at arbitrary k-points," Nature Communications, vol. 9, no. 1, May 2018.

[114] M. Gorlach and P. A. Belov, "Effect of spatial dispersion on the topological transition in metamaterials," Phys. Rev. B, vol. 90, p. 115136, Sep 2014.

[115] K. Y. Bliokh, D. Leykam, M. Lein, and F. Nori, "Topological nonhermitian origin of surface maxwell waves," Nature Communications, vol. 10, no. 1, Feb. 2019.

[116] M. G. Silveirinha, "Topological theory of non-hermitian photonic systems," Phys. Rev. B, vol. 99, p. 125155, Mar 2019.

[117] D. Colton and R. Kress, Inverse acoustic and electromagnetic scattering theory. Cham: Springer, 2019.

[118] W. C. Chew, Waves and fields in inhomogenous media. Wiley-IEEE, 1999

[119] J.-C. Nedelec, Acoustic and electromagnetic equations: integral representations for harmonic problems. New York: Springer, 2001.

[120] S. Hassani, Mathematical physics: a modern introduction to its foundations. Cham: Springer, 2013.

[121] S. Mikki and Y. Antar, New Foundations for Applied Electromagnetics: The Spatial Structure of Fields. London: Artech House, 2016.

[122] — "The antenna current Green's function formalism-Part I," IEEE Trans. Antennas Propagat, vol. 9, pp. 4493-4504, September 2013.

[123] — "The antenna current Green's function formalism-Part II," IEEE Trans. Antennas Propagat, vol. 9, pp. 4505-4519, September 2013.

[124] S. Mikki, "The antenna spacetime system theory of wireless communications," Proceedings of the Royal Society A: Mathematical, Physical and Engineering Sciences, April 2019.

[125] W. Geyi, Foundations of Applied Electrodynamics. Chichester, West Sussex Hoboken, N.J: Wiley, 2010.

[126] L. D. Landau and E. Lifshitz, Electrodynamics of continuous media. Oxford England: Butterworth-Heinemann, 1984.

[127] L. Felsen, Radiation and scattering of waves. Piscataway, NJ: IEEE Press, 1994.

[128] J. Jackson, Classical electrodynamics. New York: Wiley, 1999.

[129] J. Jin, The finite element method in electromagnetics. Hoboken. New Jersey: John Wiley \& Sons Inc, 2014

[130] A. Taflove, Computational electrodynamics: the finite-difference timedomain method. Boston: Artech House, 2005.

[131] W. C. Gibson, The Method of Moments in Electromagnetics. CRC Press, 2015.

[132] S. Mikki and A. Kishk, "Nonlocal electromagnetic media: A paradigm for material engineering," in Passive Microwave Components and Antennas. InTech, April 2010.

[133] V. M. Agranovich, Y. R. Shen, R. H. Baughman, and A. A. Zakhidov, "Linear and nonlinear wave propagation in negative refraction metamaterials," Phys. Rev. B, vol. 69, p. 165112, April 2004.

[134] V. M. Agranovich, Y. N. Gartstein, and A. A. Zakhidov, "Negative refraction in gyrotropic media," Phys. Rev. B, vol. 73, p. 045114, Jan 2006

[135] K. Cho, "A single susceptibility scheme of macroscopic maxwell equations: beyond the 'E,D,B,H' approach," Journal of Physics: Condensed Matter, vol. 20, no. 17, p. 175202, April 2008.
[136] D. B. Melrose and R. C. McPhedran, Electromagnetic processes in dispersive media: a treatment based on the dielectric tensor. Cambridge England New York: Cambridge University Press, 1991.

[137] V. Ginzburg, "Electromagnetic waves in isotropic and crystalline media characterized by dielectric permittivity with spatial dispersion," JETP, vol. 7, no. 6, p. 1096, December 1958.

[138] J. J. Hopfield, "Theory of the contribution of excitons to the complex dielectric constant of crystals," Phys. Rev., vol. 112, pp. 1555-1567, Dec 1958.

[139] S. Pekar, "The theory of electromagnetic waves in a crystal in which excitons are produced," JETP, vol. 6, no. 4, p. 785, May 1957.

[140] V. M. Agranovich and V. L. Ginzburg, "Crystal optics with allowance for spatial dispersion: Exciton theory - Part I," Soviet Physics Uspekhi, vol. 5, no. 2, pp. 323-346, feb 1962

[141] — - "Crystal optics with allowance for spatial dispersion: Exciton theory - Part II," Soviet Physics Uspekhi, vol. 5, no. 4, pp. 675-710, April 1963

[142] V. L. Ginzburg, "The development of crystal optics with allowance for spatial dispersion," Soviet Physics Uspekhi, vol. 15, no. 6, pp. 839-841, jun 1973.

[143] P. A. Belov and C. R. Simovski, "On the low-frequency spatial dispersion in wire media," in IWAT 2005. IEEE International Workshop on Antenna Technology: Small Antennas and Novel Metamaterials, 2005., March 2005, pp. 363-366.

[144] B. M. Wells, A. V. Zayats, and V. A. Podolskiy, "Nonlocal optics of plasmonic nanowire metamaterials," Phys. Rev. B, vol. 89, p. 035111 , Jan 2014.

[145] S. Mikki and A. Kishk, "Mean-field electrodynamic theory of aligned carbon nanotube composites," IEEE Transactions on Antennas and Propagation, vol. 57, no. 5, pp. 1412-1419, May 2009.

[146] A. V. Chebykin, M. A. Gorlach, A. Gorlach, and P. A. Belov, "Spatial dispersion in metamaterials based on three-dimensional arrays of spheres and disks," in 2015 Days on Diffraction (DD), May 2015, pp. 1-5.

[147] Y. Guan, L.-X. Zhong, K.-H. Chew, H. Chen, Q. Wu, and R. P. Chen, "Evolution of cos-gaussian beams in a strongly nonlocal nonlinear medium," Progress In Electromagnetics Research, vol. 141, pp. 403414, 2013

[148] J. M. McMahon, S. K. Gray, and G. C. Schatz, "Nonlocal optical response of metal nanostructures with arbitrary shape," Phys. Rev. Lett., vol. 103, p. 097403, Aug 2009.

[149] E. Ciecierska, A. Boczkowska, and K. J. Kurzydlowski, "Quantitative description of the spatial dispersion of carbon nanotubes in polymeric matrix,” Journal of Materials Science, vol. 45, no. 9, pp. 2305-2310, Jan 2010.

[150] J. S. Gomez-Diaz, J. R. Mosig, and J. Perruisseau-Carrier, "Effect of spatial dispersion on surface waves propagating along graphene sheets," IEEE Transactions on Antennas and Propagation, vol. 61, no. 7, pp. 3589-3596, July 2013

[151] P. Rodriguez-Lopez, W. J. M. Kort-Kamp, D. A. R. Dalvit, and L. M. Woods, "Nonlocal optical response in topological phase transitions in the graphene family," Phys. Rev. Materials, vol. 2, p. 014003, Jan 2018.

[152] G. W. Hanson, "Drift-diffusion: A model for teaching spatial-dispersion concepts and the importance of screening in nanoscale structures,' IEEE Antennas and Propagation Magazine, vol. 52, no. 5, pp. 198207, Oct 2010

[153] R. J. Churchill and T. G. Philbin, "Electromagnetic reflection, transmission, and energy density at boundaries of nonlocal media," Phys Rev. B, vol. 94, p. 235422, Dec 2016

[154] J. Kelley, General topology. Mineola, New York: Dover Publications, Inc, 2017.

[155] M. Hirsch, Differential topology. New York: Springer-Verlag, 1976

[156] B. I. Dundas, A short course in differential topology. Cambridge, United Kingdom New York, NY: Cambridge University Press, 2018.

[157] R. E. Showalter, Hilbert space methods in partial differential equations. Mineola, N.Y: Dover Publications, 2010.

[158] E. Zeidler, Applied functional analysis: applications to mathematical physics. Springer-Verlag, 1995.

[159] E. Hebey, Nonlinear analysis on manifolds: Sobolev spaces and inequalities. New York, N.Y. Providence, R.I: Courant Institute of Mathematical Sciences American Mathematical Society, 2000.

[160] T. Mackay and A. Lakhtakia, Electromagnetic anisotropy and bianisotropy: a field guide. Singapore Hackensack, NJ: World Scientific Publishing Co. Pte. Ltd, 2019.

[161] J. Lee, Introduction to smooth manifolds. New York London: Springer, 2012 .

[162] A. Mukherjee, Differential topology. Cham: Birkhauser, 2015. 
[163] W. Appel, Mathematics for physics and physicists. Princeton, N.J: Princeton University Press, 2007.

[164] D. Kahn, Introduction to global analysis. Mineola, N.Y: Dover Publications, 2007.

[165] J. Nestruev, Smooth manifolds and observables. Cham, Switzerland: Springer, 2020.

[166] H. Edelsbrunner, Computational topology: an introduction. Providence, R.I: American Mathematical Society, 2010.

[167] J. Frenkel, "On the transformation of light into heat in solids - Part I," Phys. Rev, vol. 37, pp. 17-44, Jan 1931.

[168] — "On the transformation of light into heat in solids - Part II," Phys. Rev., vol. 37, pp. 1276-1294, May 1931.

[169] G. H. Wannier, "The structure of electronic excitation levels in insulating crystals," Phys. Rev., vol. 52, pp. 191-197, Aug 1937.

[170] J. J. Hopfield and D. G. Thomas, "Theoretical and experimental effects of spatial dispersion on the optical properties of crystals," Phys. Rev., vol. 132, pp. 563-572, Oct 1963.

[171] A. Quattropani and W. Czaja, "Quantum theory of exciton-polaritons with spatial dispersion," Physica Scripta, vol. T29, pp. 162-166, Jan 1989.

[172] M. Bamba and H. Ishihara, "Qed of excitons with nonlocal susceptibility in arbitrarily structured dielectrics," Phys. Rev. B, vol. 78, p. 085109, Aug 2008.

[173] A. S. Davydov, Theory of molecular excitons. New York: Plenum Press, 1971.

[174] K. Cho et al., Excitons. Berlin, Heidelberg: Springer Berlin Heidelberg, 1979

[175] K. Lagoudakis, The physics of exciton-polariton condensates. Lausanne, Switzerland Boca Raton, FL: EPFL Press,Distributed by CRC Press, 2013.

[176] M. Kira and S. W. Koch, Semiconductor quantum optics. Cambridge, UK New York: Cambridge University Press, 2012.

[177] D. Bohm and D. Pines, "A collective description of electron interactions - Part I: Magnetic interactions," Phys. Rev., vol. 82, pp. 625-634, Jun 1951.

[178] D. Pines and D. Bohm, "A collective description of electron interactions - Part II: Collective vs individual particle aspects of the interactions," Phys. Rev., vol. 85, pp. 338-353, Jan 1952.

[179] R. Mattuck, A guide to Feynman diagrams in the many-body problem. New York: Dover Publications, 1992.

[180] D. Pines, The many-body problem. Reading, Mass: Addison-Wesley, 1997.

[181] _ Elementary excitations in solids: lectures on protons, electrons, and plasmons. Reading, Mass: Advanced Book Program, Perseus Books, 1999.

[182] G. Dresselhaus, "Effective mass approximation for excitons," Journal of Physics and Chemistry of Solids, vol. 1, no. 1-2, pp. 14-22, Sep. 1956.

[183] D. C. Mattis and J. P. Gallinar, "What is the mass of an exciton?" Phys. Rev. Lett., vol. 53, pp. 1391-1393, Oct 1984.

[184] A. Davydov and V. Enolskii, "Effective mass of the Pekar polaron," JETP, vol. 67, no. 2, p. 313, February 1988.

[185] A. A. Balakin, M. A. Balakina, G. V. Permitin, and A. I. Smirnov, "Wave beams in inhomogeneous anisotropic and gyrotropic media," Radiophysics and Quantum Electronics, vol. 50, no. 12, pp. 955-972, Dec 2007.

[186] O. S. Kushnir and L. O. Lokot, "Features of the optical response of dielectric crystals with incommensurate phases," Physics of the Solid State, vol. 43, no. 5, pp. 818-822, May 2001.

[187] A. Zomorodian, Topology for computing. Cambridge: Cambridge University Press, 2009.

[188] Jiafu Wang, Shaobo Qu, Jiequ Zhang, and Hua Ma, "Spatial-temporal dispersion engineering of longitudinally coupled spoof surface plasmon polaritons for free-space EM wave modulation," in 2016 Progress in Electromagnetic Research Symposium (PIERS), Aug 2016, pp. 15711572.

[189] S. Mikki and Y. Antar, "On electromagnetic radiation in nonlocal environments: Steps toward a theory of near field engineering," in 2015 9th European Conference on Antennas and Propagation (EuCAP), April 2015, pp. 1-5.

[190] S. Mikki and A. Kishk, "Effective medium theory for carbon nanotube composites and their potential applications as metamaterials," in 2007 IEEE/MTT-S International Microwave Symposium, June 2007, pp. $1137-1140$.

[191] S. Mikki, "Exact derivation of the radiation law of antennas embedded into generic nonlocal metamaterials: A momentum-space approach," in 2020 14th European Conference on Antennas and Propagation (EuCAP), 2020, pp. 1-5.

[192] S. Mikki, "Theory of electromagnetic radiation in nonlocal metamaterials - Part I: Foundations," Progress In Electromagnetics Research B, vol. 89, pp. 63-86, 2020.

[193] _ - "Theory of electromagnetic radiation in nonlocal metamaterials - Part II: Applications," Progress In Electromagnetics Research B, vol. 89, pp. 87-109, 2020.

[194] R. Geroch, Infinite-dimensional manifolds. Montreal: Minkowski Institute Press, 2013. 